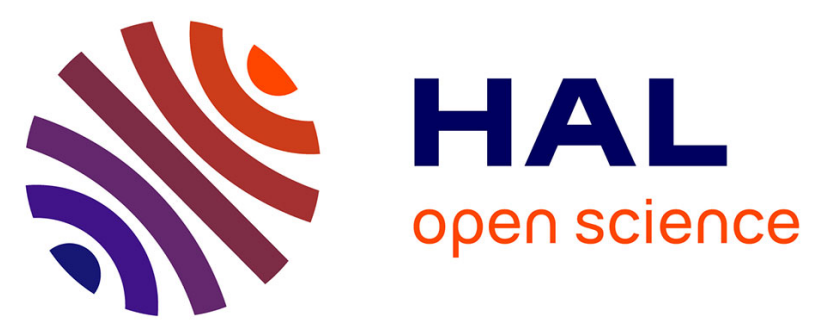

\title{
Genetic deficiency of Indoleamine 2,3-dioxygenase promotes gut microbiota-mediated metabolic health
}

Ludivine Laurans, Nicolas Venteclef, Yacine Haddad, Mouna Chajadine, Fawaz Alzaid, Sarvenaz Metghalchi, Bruno Sovran, Raphael Denis, Julien Dairou, Marina Cardellini, et al.

\section{To cite this version:}

Ludivine Laurans, Nicolas Venteclef, Yacine Haddad, Mouna Chajadine, Fawaz Alzaid, et al.. Genetic deficiency of Indoleamine 2,3-dioxygenase promotes gut microbiota-mediated metabolic health. Nature Medicine, 2018, 24 (8), pp.1113-1120. 10.1038/s41591-018-0060-4 . hal-01906028

\section{HAL Id: hal-01906028 \\ https://hal.sorbonne-universite.fr/hal-01906028}

Submitted on 26 Oct 2018

HAL is a multi-disciplinary open access archive for the deposit and dissemination of scientific research documents, whether they are published or not. The documents may come from teaching and research institutions in France or abroad, or from public or private research centers.
L'archive ouverte pluridisciplinaire HAL, est destinée au dépôt et à la diffusion de documents scientifiques de niveau recherche, publiés ou non, émanant des établissements d'enseignement et de recherche français ou étrangers, des laboratoires publics ou privés. 
3

4

5

6

7

8

\section{Genetic deficiency of Indoleamine 2, 3-dioxygenase promotes gut microbiota-mediated metabolic} health

Ludivine Laurans ${ }^{1}$, Nicolas Venteclef ${ }^{2}$, Yacine Haddad ${ }^{1}$, Mouna Chajadine ${ }^{1}$, Fawaz Alzaid ${ }^{2}$, Sarvenaz Metghalchi ${ }^{1}$, Bruno Sovran ${ }^{3}$, Raphael GP Denis ${ }^{4}$, Julien Dairou ${ }^{5}$, Marina Cardellini ${ }^{6}$, Jose-Maria Moreno-Navarrete ${ }^{7}$, Marjolene Straub ${ }^{8}$, Sarah Jegou ${ }^{8}$, Claire McQuitty ${ }^{8}$, Thomas Viel ${ }^{1}$, Bruno Esposito ${ }^{1}$, Bertrand Tavitian ${ }^{1}$, Jacques Callebert ${ }^{9}$, Serge Luquet ${ }^{4}$, Massimo Federici ${ }^{6}$, José Manuel Fernandez-real $^{7}$, Remy Burcelin ${ }^{10}$, Jean-Marie Launay ${ }^{9}$, Alain Tedgui ${ }^{1}$, Ziad Mallat ${ }^{1,11}$, Harry Sokol $^{3,8,12}$, Soraya Taleb ${ }^{1}$

${ }^{1}$ Institut National de la Santé et de la Recherche Médicale (INSERM), Unit 970, Paris Cardiovascular Research Center, and Université Paris-Descartes, Paris, France

${ }^{2}$ Institut National de la Santé et de la Recherche Médicale (INSERM) UMRS 1138, Sorbonne Universités, UPMC Université Paris 06; Sorbonne Paris Cité, Université Paris Descartes, Université Paris Diderot; and Centre de Recherche des Cordeliers, Paris, France

${ }^{3}$ Micalis Institute, Institut National de la Recherche Agronomique (INRA), AgroParisTech, Université Paris-Saclay, Jouy-en-Josas, France

${ }^{4}$ Unité de Biologie Fonctionnelle et Adaptative, Centre National la Recherche Scientifique, UMR 8251, Université Paris Diderot, Sorbonne Paris Cité, 75205 Paris, France

${ }^{5}$ UMR 8601 CNRS, Laboratoire de Chimie et Biochimie Pharmacologiques et Toxicologiques, Université Paris Descartes-Sorbonne Paris Cité, 75270, Paris, France

${ }^{6}$ Department of Systems Medicine, University of Rome Tor Vergata, Rome, Italy

${ }^{7}$ Department of Diabetes and Endocrinology. Hospital de Girona "Dr Josep Trueta", Girona, Spain; and CIBERobn Pathophysiology of Obesity and Nutrition, Instituto de Salud Carlos III, Madrid, Spain

${ }^{8}$ Sorbonne Université, École normale supérieure, PSL Research University, CNRS, INSERM, AP-HP, Hôpital Saint-Antoine, Laboratoire de biomolécules, LBM, F-75005 Paris, France

${ }^{9}$ Assistance Publique Hôpitaux de Paris, Service de Biochimie and INSERM U942, Hôpital Lariboisière, Paris, France

${ }^{10}$ Institut des maladies métaboliques et cardiovasculaires, INSERM U1048 F-31432 Toulouse, France

${ }^{11}$ Division of Cardiovascular Medicine, University of Cambridge, Addenbrooke's Hospital, Cambridge, CB2 2QQ, UK

${ }^{12}$ Department of Gastroenterology, Saint Antoine Hospital, Assistance Publique - Hopitaux de Paris, Sorbonne Université, Paris, France

\footnotetext{
* Correspondence to: Dr Soraya Taleb, PhD, at INSERM 970, 56 rue Leblanc 75015 Paris France. E-
} Mail: soraya.taleb@inserm.fr 
Abstract

The association between altered gut microbiota, intestinal permeability, inflammation and cardiometabolic diseases is becoming increasingly clear but remains poorly understood ${ }^{1,2}$. Indoleamine 2, 3-dioxygenase (IDO) is an enzyme induced in many types of immune cells including macrophages in response to inflammatory stimuli, and catalyses the degradation of tryptophan (Trp) along the kynurenine (Kyn) pathway. IDO activity is better known for its suppression of effector $\mathrm{T}$-cell immunity and its activation of regulatory $\mathrm{T}$ cells ${ }^{3,4}$. However, high IDO activity predicts worse cardiovascular outcome ${ }^{5-9}$ and may promote atherosclerosis and vascular inflammation ${ }^{6}$, suggesting a more complex role in chronic inflammatory settings. IDO activity is also increased in obesity ${ }^{10-13}$. Yet, the role of IDO in metabolic disease is still unexplored. Here we show that obesity is associated with an increase of intestinal IDO activity, which shifts Trp metabolism from indole derivative and interleukin (IL)-22 production towards Kyn production. IDO deletion or inhibition improves insulin sensitivity, preserves gut mucosal barrier, decreases endotoxaemia and chronic inflammation, and regulates lipid metabolism in liver and adipose tissues. These beneficial effects are due to rewiring of Trp metabolism towards a microbiota-dependent production of IL-22 and are abrogated after treatment with a neutralizing anti-IL-22 antibody. In summary, we identify an unexpected function of IDO in the fine tuning of intestinal Trp metabolism with major consequences on microbiota-dependent control of metabolic disease, which suggests IDO as a potential therapeutic target.

To address the role of IDO in obesity, we used high fat diet (HFD) to promote metabolic disease in wild-type (WT) and Ido1 ${ }^{-1}$ mice. HFD -fed WT mice compared to those on a normal chow diet (NCD) induced Ido1 mRNA in epididymal (epi) and inguinal (ing) white adipose tissue (WAT) as well as in soleus muscle (Supplementary Fig. 1a), whereas no Ido1 mRNA was detected in liver (data not shown). Of note, IFN- $\gamma$, known as a potent inducer of IDO $^{14}$ was also higher in the HFD-fed mice compared to those on a NCD (Supplementary Fig. 1b). Accordingly, we found that HFD-feeding resulted in significantly greater IDO activity (as assessed by measurement of Kyn/Trp ratio) in plasma, epiWAT, brown adipose tissue (AT) and the soleus muscle, in comparison to WT mice on a NCD (Fig. 1a).

Ido1 ${ }^{-/}$mice fed a NCD showed no major differences in body mass, adiposity and insulin sensitivity compared to WT on the same diet (Supplementary Fig. 2). Interestingly, when put on a HFD, Ido $1^{-/}$ mice still had a similar weight curve as WT or Ido1 $1^{-/}$mice fed a NCD (Fig. 1b) and had a lower fat mass as evaluated by magnetic resonance imaging (MRI), as compared to HFD-fed WT mice (Fig. 1c), without any change in lean mass (Supplementary Fig. 3a). In particular, the weights of epiWAT, inguinal (ing) WAT and retroperitoneal (ret) WAT were lower in HFD-fed Ido ${ }^{-/}$mice compared to HFD-fed WT mice (Fig. 1d). Consistent with lower adiposity and plasma leptin levels ${ }^{15}$ (Fig. 1e), HFD-fed $I d o 1^{-/-}$mice compared to WT on the same diet also had lower liver weights (Fig. 1f), and their livers were characterized by less lipid accumulation (Fig. 19), and lower macrophage infiltration (Supplementary Fig. 3b), indicating a protection from liver steatosis.

Obesity is known to contribute to the development of adipose tissue inflammation leading to insulin resistance $^{16,17}$. Examination of ingWAT and epiWAT revealed less macrophage infiltration (Fig. 1h), and higher content of CD11b+F4/80+CD206+ M2-like macrophages in epiWAT (Fig. 1i), with no change of $\mathrm{CD} 11 \mathrm{~b}+\mathrm{F} 4 / 80+\mathrm{CD} 11 \mathrm{c}+\mathrm{M} 1$-like (data not shown), in HFD-fed $I d o 1^{-/-}$mice compared to WT on the same diet. Similarly, epiWAT explants from HFD-fed $I d o 1^{-/-}$mice produced higher levels of protective adiponectin ${ }^{18}$ (Fig. 1j) compared to explants from WT mice on the same diet, whereas ingWAT produced higher type 2 immune cytokines IL-10, IL-4 and IL-5 (ref. 17) (Supplementary Fig. 3c), indicating a lower inflammatory status in adipose tissues of HFD-fed Ido $1^{-1 /}$ mice compared to HFD-fed WT. Consistent with lower fat mass, insulin concentrations were lesser in fasting HFD-fed 
Ido ${ }^{-/}$mice compared to WT on the same diet and during oral glucose tolerance test (OGTT) (Supplementary Fig. 3d). HFD-fed $I d o 1^{-/}$mice also showed improved insulin tolerance test (ITT) (Fig. 1k), lower AUC insulin/AUC glucose (Fig. 1l) and better insulin signalling (P-AKT) in the soleus muscle (Fig. 1m), but not in liver, ingWAT and epiWAT (Supplementary Fig. 3e), compared to HFD-fed WT mice. These results indicated that HFD-fed Ido $1^{-/}$mice were protected from obesity and related metabolic complications, including liver steatosis and insulin resistance.

To explain the weight differences between HFD-fed $I d o 1^{-/}$mice and their controls, we performed detailed metabolic analyses. Covariate analysis of relationship between body weight and energy expenditure revealed a significant difference between HFD-fed WT and HFD-fed Ido $1^{-/}$mice pointing towards a higher metabolic efficiency in absence of IDO without any change in food intake and total energy excretion (Supplementary Fig. 3f-h), and any difference in spontaneous locomotor activity or preferential substrate use (data not shown). To determine which tissues contributed to the higher energy expenditure, we used positron emission tomography-computed tomography (PET-CT). ${ }^{18} \mathrm{~F}$ fluorodeoxyglucose (FDG) uptake was higher in the muscle of HFD-fed $I d o 1^{-1-}$ mice compared to HFD-fed WT, without any observed differences in brain, brown AT and heart (Fig. 1n). This is in agreement with higher membrane glucose transporter type 4 (GLUT4) expression, mitochondrial marker staining, and greater adenosine triphosphate (ATP) production in soleus muscle of HFD-fed Ido $1^{-/}$mice compared to HFD-fed WT mice (Supplementary Fig. 4), suggesting a higher muscular metabolic rate.

Then, we sought to inhibit IDO activity using L-1Methyl Tryptophan (1MT) in drinking water. We found no differences in body weight in 1MT- treated WT mice compared to untreated mice fed with a HFD (Supplementary Fig. 5a) that may due to several factors such as the duration, extent and sustainability of IDO inhibition. However, HFD-fed WT mice treated with 1MT showed lower plasma IDO activity (as measured by the Kyn/Trp ratio), a higher production of adiponectin by epiWAT explants, improved insulin tolerance, and a lower insulin-resistance index (HOMA-IR), compared to untreated WT mice (Supplementary Fig. 5b-e). We observed similar results in genetically obese leptin-deficient $(o b / o b)$ mice treated with 1MT compared to untreated ob/ob mice (Supplementary Fig. 5f-g), indicating that the inhibition of IDO activity improved insulin resistance in obesity.

IDO is expressed by both myeloid and non-myeloid compartments ${ }^{4,19}$. To distinguish between the roles of IDO in those compartments, we generated chimeric mice. Reconstitution of WT mice with bone marrow from $\mathrm{Ido}^{-/}$mice compared to bone marrow from WT mice only slightly affected plasma IDO activity (i.e., the Kyn/Trp ratio) (Fig. 2a), mouse body weight, WAT weights and insulin sensitivity (Fig. 2b-d). Moreover, mice deleted for IDO in macrophages (Ido $1^{\text {flox/flox }}$ LysM-cre), the main cells that express IDO in the myeloid compartment ${ }^{14}$, showed similar weight curves and insulin sensitivity, compared to HFD-fed $I d o 1^{\text {flox/flox }}$ control mice, indicating that IDO in myeloid compartment is dispensable for obesity and insulin-resistance (Supplementary Fig. 6). Interestingly, mice deficient for IDO in non-myeloid cells had a marked lower plasma IDO activity (Kyn/Trp) (Fig. 2a), gained less body weight on HFD and had lower ingWAT, epiWAT, retWAT and liver weights (Fig. 2b-c), as well as improved insulin tolerance and glucose homeostasis (Fig. 2d-e), compared to HFD-fed WT mice transplanted with WT bone marrow. The results strongly support the importance of IDO expressed in non-myeloid compartment in the induction of metabolic disease.

Increased gut-derived lipopolysaccharide (LPS) translocation and intestinal dysbiosis were observed in obesity $^{20}$. Since IDO is expressed in the gastrointestinal tract $^{19}$, we analyzed intestinal IDO activity during HFD feeding. HFD feeding resulted in a markedly greater IDO activity (as measured by changes in the Kyn/Trp ratio) in both the small intestine and colon (Fig. 2f). We therefore hypothesised that intestinal IDO activity may hijack local Trp metabolism and shift it away from use by the gut microbiota. 
To address the importance of the microbiota, we depleted the gut microbiota in HFD- fed WT and $I d o 1^{-/}$mice using a broad spectrum antibiotic cocktail supplemented in drinking water. In agreement with a previous study ${ }^{21}$, depletion of the microbiota protected the mice against HFD-induced weight gain (Fig. 2g). Moreover, antibiotic treatment abrogated the differences of body weight previously seen between HFD-fed WT and HFD-fed Ido1 $1^{-/}$mice (Fig. 2g). To test whether the gut microbiota is involved in the phenotype, WT and $I d o 1^{-/-}$mice were co-housed after weaning (mix) and compared to mice housed in cages separated by genotype. As shown in Fig. 2h, the weight of co-housed animals (whether WT or $I d o 1^{-/}$) was similar to those of $I d o 1^{-/-}$mice housed in separate cages, indicating a dominant protective effect against weight gain of microbiota from $I d o 1^{-/-}$mice. Moreover, antibiotic treatment and co-housing abrogated the genotype-related differences in HOMA-IR (Fig. 2i).

We then sought to explore whether microbiota transfer might suffice to recapitulate the phenotype observed in HFD-fed Ido $1^{-/}$mice. We thus forced-fed WT mice with feces collected from $o b / o b$ mice treated or not with $1 \mathrm{MT}$. We used $o b / o b$ mice because they are already obese and they showed improved insulin sensitivity but no difference in body weight in response to 1MT treatment (data not shown), in association with a significant lower Kyn/Trp ratio in the feces (Fig. 2j). As shown in Fig. $2 \mathbf{k}-\mathbf{n}$, repetitive gavage of WT mice with feces from $1 \mathrm{MT}$-treated $o b / o b$ mice led to a lower increase of total body, WAT and liver weights, to a higher content of M2-like macrophages in epiWAT, and a lower HOMA-IR, compared to WT mice transferred with feces from control ob/ob mice, indicating protective effects of microbiota collected from mice treated with IDO inhibitor.

We next explored the bacterial fecal composition of the microbiota by use of 16S rDNA sequencing. Principal component analysis (PCA) on the basis of genus composition revealed major differences between WT and $I d o 1^{-/}$mice fed with HFD (Fig. 3a) and between $o b / o b$ mice treated or not with 1MT (Supplementary Fig. 7a). No differences regarding bacterial biodiversity were observed between WT and $I d o 1^{-/}$mice fed with HFD, and between $o b / o b$ mice treated or not with 1MT (Supplementary Fig. 7b). At the phylum level, important differences were observed between WT and Ido1 ${ }^{-1-}$ mice fed with either a NCD or a HFD (Fig. 3b). In particular, we found that the HFD led to higher Firmicutes to Bacteroidetes ratio in WT mice, as previously reported ${ }^{22}$, whereas HFD-fed $I d o 1^{-/}$mice showed a reduction of this ratio, compared to NCD-fed $\mathrm{Ido}^{-/}$mice (Fig. 3b). At the family level, significantly greater proportions of Ruminococcaeae and lower proportions of Rikenellaceae were observed in HFD-fed WT mice compared to NCD-fed WT mice (Fig. 3c), in agreement with previous reports ${ }^{23,24}$. Whereas in HFD-fed Ido ${ }^{-/}$mice compared to NCD-fed Ido $1^{-1-}$ mice, the decrease of Firmicutes was mainly due to a lower proportion of Clostridiales, in particular Lachnospiraceae (Fig. 3c and Supplementary Fig. 7c). The decrease of Lachnospiraceae was also observed on 1MT-treated ob/ob mice compared to untreated mice (Supplementary Fig. 7d). Moreover, a positive correlation was observed between the proportion of Clostridiales lachnospiraceae in feces and LPS levels in plasma (Supplementary Fig. 7e), suggesting a beneficial impact of the decrease of a selective bacterial species on inflammation in HFD-fed $I d o 1^{-/}$and $1 \mathrm{MT}$-treated $o b / o b$ mice. These results were confirmed, using the linear discriminant analysis (LDA) effect size (LEFSE) pipeline comparing HFD-fed WT and HFD-fed Ido1 $1^{-/}$mice (Supplementary Fig. 7f). Overall, these data demonstrate that IDO has an important role in shaping gut microbiota, which is required to control body weight and insulin-resistance.

We next examined whether Kyn or derived metabolites played a direct role in obesity, as previously suggested $^{25}$. In particular, administration of kynurenic acid (Kna), a metabolite downstream of Kyn, to WT mice has been shown to activate G protein-coupled receptor (GPR) 35 and rises energy expenditure ${ }^{26}$. To this end, we supplemented $I d o 1^{-/}$mice with Kyn or Kna added in drinking water. Kyn supplementation in Ido1 $1^{-/}$mice did not change body weight, WAT weights or insulin sensitivity despite a higher plasma Kyn levels (Supplementary Fig. 8). Moreover, Kna supplementation in Ido1 - mice did not alter body weight (data not shown). Our results indicate that the absence of Kyn or derived metabolites in $\mathrm{Ido1}^{-/}$mice does not explain the observed protection against metabolic disease. 
Trp is either metabolized by IDO to produce Kyn or by gut bacteria into indole derivatives, such as indole-3-acetic acid (IAA) that activates aryl hydrocarbon receptor (AHR) ${ }^{27}$ (Supplementary Fig. 9a). We hypothesised that in obesity the increase of IDO activity shifts Trp metabolism from generation of indole derivatives towards Kyn production. To test this, we examined intestinal content of IAA, Trp and Kyn in NCD or HFD-fed WT or Ido $1^{-/}$mice, $o b / o b$ mice treated or not with 1MT, and in WT mice that received feces from 1MT-treated or untreated ob/ob mice. As shown in Fig. 3d, HFD in WT mice led to lower intestinal content of IAA, whereas it markedly induced Kyn levels in the gastrointestinal tract, indicating that HFD-induced obesity causes a major shift of Trp metabolism towards Kyn production. Consistently, in the case of a low level of intestinal Kyn as in HFD-fed Ido1 '- mice (Fig. 3d) and in 1MT-treated ob/ob mice (Supplementary Fig. 9b), a substantially higher IAA intestinal content was observed, as compared with HFD-fed WT mice (Fig. 3d) and control ob/ob mice (Supplementary Fig.9c), without major changes of intestinal Trp levels (Supplementary Fig. 9d-e). Moreover, a higher intestinal IAA was observed in WT mice that received feces from 1MTtreated mice compared to non-treated mice (Supplementary Fig. 9f), indicating the importance of IDO-dependent changes of microbiota in IAA production. Using an AHR reporter system, we found that small intestines contents of HFD-fed Ido1 ${ }^{-/}$mice activated AHR more than those recovered from HFD-fed WT mice (Supplementary Fig. 9g). This data supports the importance of IDO in controlling Kyn and IAA-activating AHR balance. Moreover, AHR activation or IAA supplementation in WT mice fed a HFD reduced insulin resistance and epiWAT inflammation (Supplementary Fig. 10a-f), without significant changes in body weight (data not shown).

We then explored the role of the 2 cytokines related to indole metabolites ${ }^{27}$, IL-17 and IL-22, in our findings. In agreement with previous reports showing that HFD diminished IL-17 and IL-22 (ref. 28, 29), we found lower levels of these cytokines in Peyer's patches (PP) of HFD-fed WT compared to NCD-fed WT mice (Fig. 3e). Moreover, in agreement with higher IAA ${ }^{27}$, we observed more IL-17 and IL-22 in HFD-fed Ido $1^{-/}$mice compared to HFD-fed WT (Fig. 3e) as well as more IL-22 in 1MTtreated WT mice compared to non-treated mice (Supplementary Fig. 10g). Furthermore, a higher intestinal IL-22 level was observed in WT mice that received feces from 1MT-treated $o b / o b$ mice compared to non-treated $o b / o b$ mice (Supplementary Fig. 10h). Intestinal IAA levels were positively correlated with intestinal IL-22 levels and negatively with HOMA-IR (Supplementary Fig. 10i-j). We further found an increase of IL-22-target genes such as antimicrobial proteins ${ }^{30}$, regenerating isletderived (Reg)3g, Reg3b mRNA (Fig. 3f) in intestines of HFD-fed $I d o 1^{-/}$compared to HFD-fed WT mice. Short-chain fatty acids (SCFAs), mainly acetate, propionate and butyrate, are the end products of fermentation of dietary fibres by the anaerobic intestinal microbiota, and have been shown to exert multiple beneficial effects ${ }^{2}$. Interestingly, a higher fecal level of SCFAs was observed in HFD-fed Ido $1^{-/}$compared to WT mice fed with the same diet (Fig. 3g) supporting a restoration of the intestinal ecosystem. Moreover, we observed lower expression of inflammation-associated genes (differentially expressed between HFD-fed WT and $I d o 1^{-/}$mice) in intestines of HFD-fed $I d o 1^{-/}$compared to HFDfed WT mice, using NanoString technology (Fig. 3h). As previously published ${ }^{20}$, we found that HFD led to a higher plasma LPS (Fig. 3i). However, HFD-fed Ido $1^{-/}$mice showed lower plasma LPS in comparison to HFD-fed WT mice (Fig. 3i), which was also the case in 1MT-treated WT and ob/ob mice compared to untreated controls (data not shown). Altogether these results provide a strong evidence for a protective role of IDO deletion in preserving intestinal immune barrier during obesity. IL-22 was shown to exert essential roles in eliciting antimicrobial immunity and maintaining mucosal barrier integrity within the intestine ${ }^{31,32}$. Given the observed higher levels of IL-22 in HFD-fed Ido $1^{-/}$ mice compared to HFD-fed WT mice, we injected mouse anti-IL-22 neutralizing antibody or control IgG1 to WT and $I d o 1^{-/}$mice during HFD period. Neutralization of IL-22 in HFD-fed Ido $1^{-/}$mice compared to HFD-fed WT mice abrogated the protective effects of IDO deletion on obesity, insulin sensitivity and intestinal permeability (Fig. 3j-o and Supplementary Fig. 11).

As rodents may differ from humans regarding the regulation of IDO activity ${ }^{33}$, we then explored the relevance of our data in the human setting of obesity (Supplementary Table). In line with the 
dysfunction of gut barrier function in obesity, we detected a higher circulating endotoxin level in subjects with obesity in comparison with non-obese individuals (Fig. 4a). Moreover, plasma Kyn level was higher in subjects with obesity or with type 2 diabetes compared to controls (Fig. 4b). We then analysed for the first time the levels of fecal Trp, Kyn and IAA in the context of human obesity. In agreement with our mouse data, we observed a shift of Trp metabolism towards more Kyn and less IAA in feces of subjects with obesity or diabetes compared to non-obese subjects (Fig. 4c). We found no correlation between plasma and feces levels of $K y n / T r p$ ratio $(r=0.04, P=0.75)$, suggesting a specific micro-environmental regulation of IDO in intestine of subjects with obesity. We then examined correlations between feces or plasma Kyn levels and metabolic and clinical parameters in subjects with obesity. We found positive correlations between plasma Kyn and body weight $(r=0.37$, $P=0.007)$, waist-circumference $(r=0.34, P=0.01)$, fat mass $(r=0.38, P=0.01)$, plasma LPS $(r=$ $0.31, P=0.02)$, aspartate aminotransferase AST $(r=0.34, P=0.02)$, area under the curve during an oral glucose tolerance test $(r=0.37, P=0.008)$, but negative correlations with HDL-cholesterol $(r=$ $0.31, P=0.03)$ and glucose rate during euglycemic hyperinsulinemic clamp $(r=-0.40, P=0.006)$. We observed an inverse correlation between feces Kyn levels and HDL-cholesterol $(r=-0.35, P=0.01)$, but detected positive correlations between feces Kyn levels and plasma triglycerides $(r=0.38, P=$ $0.007)$, and triglycerides/HDL-cholesterol ratio $(r=0.42, \quad P=0.002)$, the latter being a surrogate marker for cardiometabolic risk ${ }^{34}$. These data indicate that high levels of Kyn in plasma and feces are associated with a deleterious metabolic profile in the setting of obesity. 
This work was supported by INSERM, Fondation pour la Recherche Médicale (A.T.) and Fondation de France (S.T.). H.S. received funding from the European Research Council (ERC) under the European Union's Horizon 2020 research and innovation programme (ERC-2016-StG-71577).We are grateful to S. Billon-Crossouard from the Platform of Mass Spectrometry of Nantes University for SCFA analysis. We acknowledge the technical platform metabolism of the Unit "Biologie Fonctionnelle et Adaptative" (University Paris Diderot, Sorbonne Paris Cité, BFA, UMR 8251 CNRS, 75205 Paris, France) for metabolic analysis and the animal core facility "Buffon" of the University Paris Diderot Paris 7/Institut Jacques Monod, Paris for animal husbandry. We are thankful to the Genomics Platform of Translational Research Department, Institut Curie, PSL Research University for sharing their expertise and helping us with Nanostring analysis. We thank members of our animal and histology Facilities. We thank B. Gaye for his help with statistical analysis. We thank N. Vodovar for helpful discussions and C. Heymes for his help for human sample preparation.

\section{Author Contribution statement}

L.L. was involved in experimental design, conducted most experiments and analyzed data. N.V. provided technical and conceptual helps on obesity experiments and discussed results. Y.H., M.C., S.M., B.S. helped in some experiments. R.G.P.D., designed, performed, analyzed and interpreted the indirect calorimetry exploration. F.A. helped with experiments and performed immunohistological staining. M.S., C.M. and S.J. provided technical help for microbiota analysis. T.V. and B.T. performed and discussed PET analysis. B.E. helped with in vivo studies. J-M.L., J.D. and J.C. measured all biochemical parameters in mouse and human samples. S.L. provided funding and contributed to calorimetry data analysis and interpretation. M.C., J-M. M-N., M.F., JM.F-R. and R.B. provided human material and clinical data. A.T. and Z.M. discussed results and edited the manuscript. H.S. performed and interpreted gut microbiota analysis, provided some of human samples and discussed results. S.T. designed the study, analyzed and interpreted the data, and wrote the manuscript.

\section{Competing financial interests}

No conflict of interest 
1. Tang, W.H., Kitai, T. \& Hazen, S.L. Gut Microbiota in Cardiovascular Health and Disease. Circ Res 120, 1183-1196 (2017).

2. Schroeder, B.O. \& Backhed, F. Signals from the gut microbiota to distant organs in physiology and disease. Nat Med 22, 1079-1089 (2016).

3. Puccetti, P. \& Grohmann, U. IDO and regulatory T cells: a role for reverse signalling and noncanonical NF-kappaB activation. Nature reviews. Immunology 7, 817-823 (2007).

4. Mellor, A.L. \& Munn, D.H. IDO expression by dendritic cells: tolerance and tryptophan catabolism. Nature reviews. Immunology 4, $762-774$ (2004).

5. Wirleitner, B., et al. Immune activation and degradation of tryptophan in coronary heart disease. Eur J Clin Invest 33, 550-554 (2003).

6. Metghalchi, S., et al. Indoleamine 2,3-Dioxygenase Fine-Tunes Immune Homeostasis in Atherosclerosis and Colitis through Repression of Interleukin-10 Production. Cell metabolism 22, 460-471 (2015).

7. Pedersen, E.R., et al. Systemic markers of interferon-gamma-mediated immune activation and long-term prognosis in patients with stable coronary artery disease. Arterioscler Thromb Vasc Biol 31, 698-704 (2011).

8. Pedersen, E.R., et al. Associations of Plasma Kynurenines With Risk of Acute Myocardial Infarction in Patients With Stable Angina Pectoris. Arterioscler Thromb Vasc Biol (2014).

9. Eussen, S.J., et al. Kynurenines as predictors of acute coronary events in the Hordaland Health Study. Int J Cardiol 189, 18-24 (2015).

10. Brandacher, G., et al. Bariatric surgery cannot prevent tryptophan depletion due to chronic immune activation in morbidly obese patients. Obesity surgery 16, 541-548 (2006).

11. Wolowczuk, I., et al. Tryptophan metabolism activation by indoleamine 2,3-dioxygenase in adipose tissue of obese women: an attempt to maintain immune homeostasis and vascular tone. Am J Physiol Regul Integr Comp Physiol 303, R135-143 (2012).

12. Mangge, H., et al. Disturbed tryptophan metabolism in cardiovascular disease. Current medicinal chemistry 21, 1931-1937 (2014).

13. Favennec, M., et al. The kynurenine pathway is activated in human obesity and shifted toward kynurenine monooxygenase activation. Obesity 23, 2066-2074 (2015).

14. Yoshida, R., Imanishi, J., Oku, T., Kishida, T. \& Hayaishi, O. Induction of pulmonary indoleamine 2,3-dioxygenase by interferon. Proc Natl Acad Sci U S A 78, 129-132 (1981).

15. Zhang, Y., et al. Positional cloning of the mouse obese gene and its human homologue. Nature 372, 425-432 (1994).

16. Hotamisligil, G.S. Inflammation and metabolic disorders. Nature 444, 860-867 (2006).

17. Odegaard, J.I. \& Chawla, A. Pleiotropic actions of insulin resistance and inflammation in metabolic homeostasis. Science 339, 172-177 (2013).

18. Yamauchi, T., et al. The fat-derived hormone adiponectin reverses insulin resistance associated with both lipoatrophy and obesity. Nat Med 7, 941-946 (2001).

19. Cherayil, B.J. Indoleamine 2,3-dioxygenase in intestinal immunity and inflammation. Inflammatory bowel diseases 15, 1391-1396 (2009).

20. Cani, P.D., et al. Metabolic endotoxemia initiates obesity and insulin resistance. Diabetes 56, 1761-1772 (2007).

21. Suarez-Zamorano, N., et al. Microbiota depletion promotes browning of white adipose tissue and reduces obesity. Nat Med 21, 1497-1501 (2015).

22. Turnbaugh, P.J., et al. An obesity-associated gut microbiome with increased capacity for energy harvest. Nature 444, 1027-1031 (2006).

23. Kim, K.A., Gu, W., Lee, I.A., Joh, E.H. \& Kim, D.H. High fat diet-induced gut microbiota exacerbates inflammation and obesity in mice via the TLR4 signaling pathway. PLoS One 7, e47713 (2012).

24. Clarke, S.F., et al. Targeting the microbiota to address diet-induced obesity: a time dependent challenge. PLoS One 8, e65790 (2013). 
25. Moyer, B.J., et al. Inhibition of the aryl hydrocarbon receptor prevents Western diet-induced obesity. Model for AHR activation by kynurenine via oxidized-LDL, TLR2/4, TGFbeta, and IDO1. Toxicology and applied pharmacology 300, 13-24 (2016).

26. Agudelo, L.Z., et al. Kynurenic Acid and Gpr35 Regulate Adipose Tissue Energy Homeostasis and Inflammation. Cell metabolism 27, 378-392 e375 (2018).

27. Lamas, B., et al. CARD9 impacts colitis by altering gut microbiota metabolism of tryptophan into aryl hydrocarbon receptor ligands. Nat Med 22, 598-605 (2016).

28. Garidou, L., et al. The Gut Microbiota Regulates Intestinal CD4 T Cells Expressing RORgammat and Controls Metabolic Disease. Cell metabolism 22, 100-112 (2015).

29. Wang, X., et al. Interleukin-22 alleviates metabolic disorders and restores mucosal immunity in diabetes. Nature 514, 237-241 (2014).

30. Sonnenberg, G.F., Fouser, L.A. \& Artis, D. Border patrol: regulation of immunity, inflammation and tissue homeostasis at barrier surfaces by IL-22. Nature immunology 12, 383-390 (2011).

31. Rutz, S., Eidenschenk, C. \& Ouyang, W. IL-22, not simply a Th17 cytokine. Immunological reviews 252, 116-132 (2013).

32. Gulhane, M., et al. High Fat Diets Induce Colonic Epithelial Cell Stress and Inflammation that is Reversed by IL-22. Scientific reports 6, 28990 (2016).

33. Murakami, Y. \& Saito, K. Species and cell types difference in tryptophan metabolism. International journal of tryptophan research : IJTR 6, 47-54 (2013).

34. Salazar, M.R., et al. Relation among the plasma triglyceride/high-density lipoprotein cholesterol concentration ratio, insulin resistance, and associated cardio-metabolic risk factors in men and women. Am J Cardiol 109, 1749-1753 (2012). 
364 Figure $1 \mathrm{Ido} 1^{-/}$mice are protected from obesity, inflammation, liver steatosis and insulin resistance. (a) IDO activity (Kyn/Trp ratio) in plasma, ingWAT, epiWAT, brown AT, liver and soleus from WT mice fed with either a HFD $(n=5)$ or a NCD $(n=5)$ or HFD-fed Ido $1^{-/-}$mice after 20 weeks. HFD-fed $I d o 1^{-/}$mice values are displayed only as controls. (b) body mass of WT and Ido $1^{-/}$mice fed with either HFD ( $n=10$ per group) or NCD ( $n=5$ per group) during 20 weeks.. This result was confirmed in a total of 4 independent experiments. (c-f) Body fat \% (c), weights in grams of epiWAT, ingWAT and retWAT (d), plasma leptin (e) and liver weight (f) of WT and Ido ${ }^{-/}$mice fed with HFD during 20 weeks. (g) Representative pictures (left) and quantification (right) of lipid areas in liver cross-sections from HFD-fed WT and $I d o 1^{-/}$mice ( $n=9$ per group). Scale bars, $100 \mu \mathrm{m}$. (h) Representative images (left) and quantifications (right) of F4/80 staining in ingWAT and epiWAT ( $n=5$ per group). Scale bars, $100 \mu \mathrm{m}$. F4/80 staining (in red) was normalized on Plin1 staining (in green). (i-l) quantification of M2-like macrophages (F4/80+CD11b+CD206 + ) in epiWAT ( $n=5$ per group) (i), adiponectin production by epiWAT explants ( $n=10$ per group) $(\mathbf{j})$, insulin tolerance test (ITT) adjusted on body weight (confirmed in a total of 3 independent experiments) (k), ratio of area under curve (AUC) of insulin to AUC glucose during oral glucose tolerance test (OGTT) in WT and Ido1 $1^{-/}$mice fed with HFD during 20 weeks ( $n=10$ per group) (l). (m) Insulin signaling (pAKT-S473) in soleus after 15 $\mathrm{min}$ of $5 \mathrm{U} / \mathrm{kg}$ insulin injection. Cropped blot images are shown, the full scans are available in Supplementary Fig. 12. The result is representative of two independent experiments. (n) Representative FDG-PET images (left) and quantification (right) of tissue FDG uptake in WT and $I d o 1^{-/}$mice fed with HFD during 14 weeks ( $n=5$ per group). SUV, standardized uptake values. White arrows show the FDG uptake in muscle. Data are expressed as mean \pm sem. Mann-Whitney test (a-l) or two-tailed unpaired Student's $t$-test (n) was used for statistical analysis. ${ }^{*} P<0.05,{ }^{* *} P<0.001$, $* * * P<0.0001$.

Figure 2 IDO activity controls gut microbiota-dependent regulation of obesity and its complications. (a-e) IDO activity (Kyn/Trp ratio) in plasma ( $n=5$ per group) (a), $\%$ of weight gain (b), weights in grams of ingWAT, epiWAT, retWAT and liver (c), insulin test tolerance (ITT) (d), oral glucose tolerance test (OGTT) (e) in WT mice irradiated and transplanted with either WT or Ido1 ${ }^{-/}$bone marrow $\left(I d o 1^{-/}->\right.$WT $(n=10)$ and WT $\rightarrow$ WT $(n=10)$ groups) or $I d o 1^{-/}$mice irradiated and transplanted with WT bone marrow (WT $->\operatorname{Ido}^{-/}(n=10)$ ) after 20 weeks of HFD. (f) IDO activity (i.e. $\mathrm{Kyn} / \mathrm{Trp})$ in small intestines and colons of WT mice fed with either a NCD $(n=5)$ or a HFD $(n=$ $4)$ and HFD-fed Ido1/- mice $(n=4)$. (g-i) weight curves (non-mixed and non-treated HFD-fed WT and $I d o 1^{-/}$mice weight curves are displayed only as controls), (g and h) HOMA-IR index normalized to body weight of WT and $I d o 1^{-/}$mice either on antibiotic treatment $(\mathrm{Ab})(n=10$ per group $)$ or WT and $I d o 1^{-/}$mice mixed in the same cages from 4 weeks of age (mix) $\left(n=8\right.$ per group) or WT and Ido ${ }^{-/-}$ mice untreated and separated in different cages $(n=10$ per group) (i). (j-n) gavage of WT mice with feces from 1MT-treated or not treated $o b / o b$ mice $(n=10$ per group). Ratio of Kyn/Trp in feces of 1MT-treated or not treated $o b / o b$ mice $(n=4$ per group) (j), body mass $(\mathbf{k})$, weights in grams of ingWAT, epiWAT, retWAT and liver (l), representative cytometry (left) and quantification (right) of M2-like macrophages (F4/80+CD11b+CD206+) in epiWAT ( $n=5$ per group) (m) and HOMA-IR in WT mice that received feces from 1MT-treated or untreated $o b / o b$ mice $(n=10$ per group). n.s., nonsignificant. Data are expressed as mean \pm sem. Mann-Whitney test was used for statistical analysis (an). $* P<0.05, * * P<0.001, * * * P<0.0001$.

Figure 3 IDO deficiency preserves the intestinal barrier through IL-22 in the setting of obesity. (a) PCA plot based on bacterial 16S rDNA gene sequence abundance in fecal content of WT and Ido $1^{-/-}$ mice fed with either a NCD or a HFD. Axes correspond to principal components 1 (x-axis), 2 (y-axis) and 3 (z-axis). (b, c) bacterial-taxon-based analysis at the phylum level (b) and at family level (c) in the fecal microbiota. (d) IAA (left) and Kyn (right) levels in small intestines and colons of WT fed with either a NCD $(n=5)$ or a HFD $(n=4)$ and HFD-fed Ido $1^{-/}$mice $(n=4)$. (e) IL-17 (left) and IL- 
22 (right) contents in Peyer's patches (PP) of WT and $I d o 1^{-/}$mice fed with either a NCD $(n=4$ per group) or a HFD ( $n=3$ per group). The result was confirmed in two independent experiments. (f) Reg3b (left) and $3 g$ (right) mRNA in intestines of HFD-fed WT $(n=3)$ and Ido1 ${ }^{-/}$mice $(n=4)$. (g) SCFA contents in the fecal microbiota from HFD-fed WT $(n=10)$ and $I d o 1^{-/ /}$mice $(n=9)$. The result is a pool of 2 independent experiments. (h) The heat map generated using the hierarchical clustering shows expression of genes differentially expressed in intestines of HFD-fed WT $(n=3)$ and Ido $1^{-1 /}$ mice $(n=4)$. (i) Plasma LPS in WT and $I d o 1^{-/}$mice fed with either NCD or HFD $(n=5$ per group) after 20 weeks. The result was confirmed in two independent experiments. (j-n) $\%$ of weight gain after 12 weeks of HFD (j), OGTT (k), ITT (I), HOMA-IR (m), liver steatosis (n) and plasma LPS (o) in WT and $I d o 1^{-/-}$mice injected with mouse anti-IL-22 neutralising antibody, 3 times per week during 12 weeks of HFD. Scale Bars 100 $\mu$ M. Data are expressed as mean \pm sem. Mann-Whitney test (d, g, $\mathbf{i}$ and $\mathbf{m})$ or two-tailed unpaired Student's $t$-test (e and $\mathbf{f}$ ) was used for statistical analysis. ${ }^{*} P<0.05,{ }^{* *} P<$ $0.001, * * * P<0.0001$.

Figure 4 A shift of Trp metabolism towards more Kyn and less IAA in the context of human obesity and type 2 diabetes. (a) plasma LPS, (b) plasma Trp (left) and Kyn (right) in subjects with obesity ( $n=$ $49)$ or type 2 diabetes $(n=43)$ and non-obese individuals $(n=20)$. (c) Trp (left), Kyn (middle) and $\mathrm{Kyn} / \operatorname{Trp}$ (right) in feces from subjects with obesity $(n=49)$ or type 2 diabetes $(n=43)$ and non-obese subjects $(n=34)$. (d) IAA contents in feces from subjects with obesity $(n=49)$ or type 2 diabetes $(n=$ $43)$ and non-obese subjects $(n=34)$. Mann-Whitney test (a) or Kruskal-Wallis (b-d) was used for statistical analysis. Data are expressed as mean \pm sem. $* P<0.05, * * * P<0.0001$.

432 
Male C57Bl $/ 6$ Ido1 $^{-/}$mice were bought from the Jackson Laboratory (Jax) and bred in our facility. At weaning, mice were separated according to the genotype. Male $o b / o b$ mice were bought from Janvier Laboratory at 4 weeks of age. To generate Ido $1^{\text {floxflox }}$ LysM-Cre, Ido $1^{\text {flox flox }}$ mice were crossed to LysM-Cre mice. Mice were fed with either a normal chow diet (NCD) (A03, SAFE, France) or subjected to diet-induced obesity containing 60\% FAT (E15742-347, SSNIFF, Germany). High fat diet (HFD) was started at 7 weeks of age and continued for 20 weeks or less with ad libitum access to water and food. For chimerism experiment, we subjected 10 weeks old C57B1/6 WT and C57B1/6 $I d o 1^{-1-}$ mice to medullar aplasia by 9.5 gray lethal total body irradiation. We repopulated the mice with an intravenous injection of bone marrow cells isolated from femurs and tibias of male C57B1/6 WT and C57B1/6 Ido $1^{-/}$mice. After 4 weeks of recovery, mice were fed a HFD for 20 weeks. In some experiments, IDO inhibitor (L-1methyl tryptophan, 1MT) (Sigma Aldrich) or IAA (indole acetic acid; Sigma Aldrich) was used at $2 \mathrm{mg} / \mathrm{ml}$ diluted in drinking water. The 6-formylindolo(3,2-b)carbazole (Ficz; Sigma Aldrich) was resuspended in dimethyl sulfoxide (DMSO; Sigma Aldrich) and administered intraperitoneally ( $1 \mathrm{ug} /$ mouse, $1-3$ times per week) during 15 weeks of HFD. In another experiment, kynurenine $\left(2 \mathrm{mg} / \mathrm{ml}\right.$ diluted in drinking water) supplementation to Ido $1^{-/}$mice was performed during 15 weeks of HFD. We also subjected some mice to antibiotic treatment as described before $^{35}$. All mice used in these experiments were bred and housed in a specific pathogen-free barrier facility. Animal experiments were performed according to the European directive (2010/63/UE) and to the institutional guidelines approved by the local ethics committee of the French authorities, the 'Comité d'Ethique en Experimentation Animale' (CEEA) under the following number 17-018.

\section{In vivo Studies}

For oral glucose tolerance test (OGTT), mice were fasted overnight prior to an oral administration of $1-5 \mathrm{~g} / \mathrm{kg}$ glucose. Blood was sampled from the tail vein at $0,5,15,30,60,90$ and $120 \mathrm{~min}$ in order to assay glucose concentration (OneTouch Ultra glucometer, LifeScan Europe). At 0, 15, 30, 60 min tail vein blood was collected, plasma samples were stored at $-20^{\circ} \mathrm{C}$ until they were analyzed for insulin concentration (Crystal Chem Inc., Downers Grove, USA). Insulin tolerance test (ITT) was performed in mice food deprived for $5 \mathrm{~h}$ prior to an intraperitonial injection of $1 \mathrm{U} / \mathrm{kg}$ insulin. Blood was sampled from the tail vein at $0,5,15,30,60$ and $90 \mathrm{~min}$ in order to assay glucose concentration. For insulin signaling assays C57Bl/6 WT and C57B1/6 Ido $1^{-/ /}$mice were fasted overnight and then treated by intraperitoneal injection with $5 \mathrm{U} / \mathrm{kg}$ insulin $(15 \mathrm{~min})$. Tissue samples were examined by immunoblot analysis by probing with antibodies to phospho-AKT, AKT (Cell Signaling). Experiments with fecal gavage were done with fresh stool samples from either $o b / o b$ control mice or $o b / o b$ mice supplemented with 1MT during 6 weeks until 19 weeks. Briefly, stool were suspended in water and sieved through a $70 \mu \mathrm{m}$ cell strainer (BD). These fecal suspensions were inoculated to C57B1/6 WT mice via oral gavage with $400 \mu \mathrm{L}$ of fecal suspension 4-5 times per week during 15 weeks of HFD. For anti-IL-22 neutralizing antibody treatment, WT and $I d o 1^{-1-}$ mice were injected intraperitoneally three times per week with mouse anti-IL22 neutralizing antibody (50 $\mu \mathrm{g} / \mathrm{mouse})$ (Genentech, South San Francisco, CA, USA) or an equivalent amount of isotype control (IgG1) (Genentech) for a period of 12 weeks of HFD.

\section{Analysis of metabolic parameters}

Blood glucose level was measured using a glucometer (OneTouch Ultra, LifeScan Europe). Plasma insulin (Crystal Chem Inc., Downers Grove, USA) and plasma leptin was determined by ELISA (R\&D Systems). HOMA-IR in mice was calculated using the equation ((fasting glucose concentration $\mathrm{x}$ fasting insulin concentration)/405) as previously described ${ }^{36}$. Areas under the curve (AUCs) for glucose and insulin were calculated for both $\mathrm{C} 57 \mathrm{Bl} / 6$ and $\mathrm{C} 57 \mathrm{Bl} / 6 \mathrm{Ido}^{-/-}$mice during the $2 \mathrm{~h}$ OGTT using the trapezoid method ${ }^{37}$. LPS in plasma was measured with a colorimetric diagnostic kit (Pierce, USA). 
Measurement of short chain fatty acids (SCFA) was performed as described previously ${ }^{38}$ with slight modifications. A stock solution of SCFA metabolites (Sigma Aldrich, France) was prepared and serially diluted to get 10 calibration solutions. A working solution of internal standards was prepared in $0.15 \mathrm{M} \mathrm{NaOH}$ to get the following final concentrations: $75 \mathrm{mmol} / \mathrm{L}$ of $\mathrm{D}_{3}$-acetate, $3.8 \mathrm{mmol} / \mathrm{L}$ of $\mathrm{D}_{5}$-propionate, $2.5 \mathrm{mmol} / \mathrm{L}$ of ${ }^{13} \mathrm{C}$-butyrate, $0.5 \mathrm{mmol} / \mathrm{L}$ of $\mathrm{D}_{9}$-valerate (Sigma Aldrich). Stool samples were weighed $(\sim 50 \mathrm{mg})$, dissolved in $200 \mu \mathrm{L}$ of sodium hydroxide solution at $0.15 \mathrm{M}(\mathrm{NaOH}$, Sigma Aldrich). Twenty microliters of the internal standard solution were added to stool samples and calibration solutions. Each sample was then acidified with $5 \mu \mathrm{L}$ of hydroxide chloride $37 \%$ (Sigma Aldrich, France) and then extracted with $1.7 \mathrm{~mL}$ of diethyl ether (Biosolve, France). Samples were stirred gently for 1 hour and then centrifuged $2 \min \left(5000 \mathrm{rpm}, 4^{\circ} \mathrm{C}\right)$. The organic layers were transferred into $1.5 \mathrm{ml}$ glass vials and SCFAs were derivatized with $20 \mu \mathrm{L}$ of tert-butyldimethylsilyl imidazole (Sigma Aldrich, France). Samples were incubated $30 \mathrm{~min}$ at $60^{\circ} \mathrm{C}$ before analysis. Samples were finally analyzed by GC-MS (model 7890A-5975C, Agilent Technologies, France) using a $30 \mathrm{~m} \times$ $0.25 \mathrm{~mm} \times 0.25 \mu \mathrm{m}$ capillary column (HP1-MS, Agilent Technologies, France). The temperature program started at $50^{\circ} \mathrm{C}$ for $1 \mathrm{~min}$, ramped to $90^{\circ} \mathrm{C}$ at $5^{\circ} \mathrm{C} / \mathrm{min}$, then up to $300^{\circ} \mathrm{C}$ at $70^{\circ} \mathrm{C} / \mathrm{min}$. Selected ion monitoring (SIM) mode was used to measure SCFA concentrations with ions at $\mathrm{m} / \mathrm{z} 117$ (acetate), 120 ( $\mathrm{D}_{3}$-acetate), 131 (propionate), 136 ( $\mathrm{D}_{5}$-propionate), 145 (butyrate and isobutyrate), 146 $\left({ }^{13} \mathrm{C}\right.$-butyrate), 159 (valerate), 168 ( $\mathrm{D}_{9^{-}}$valerate).

\section{Immunohistochemical analyses}

Liver sections $(7 \mu \mathrm{m})$ were used for Oil Red $\mathrm{O}$ coloration to visualize lipid accumulation and to perform CD68 staining. Briefly, sections were fixed $5 \mathrm{~min}$ in PFA prior to staining. Macrophages were detected using anti-CD68 antibody (AbD Serotec MCA1975) overnight at $4^{\circ} \mathrm{C}$. Anti-Rat peroxydase polymer (Histofine) and IHC chromogen substrate (Thermo scientific) were used for revelation. Immediately after collection, adipose tissue samples were drop-fixed in formaldehyde solution for 24 hours, then put through an automated carousel processor for dehydration, clearing and paraffin embedding (Leica). Briefly, tissue sections were dewaxed through three consecutive incubations (5 $\mathrm{min}$ ) in xylene and then dehydrated by three consecutive incubations ( $3 \mathrm{~min}$ ) in $96-100 \%$ ethanol, followed by 2 washes $(2 \mathrm{~min})$ in de-ionized water. Sections were then incubated for $15 \mathrm{~min}$ at room temperature in $3 \%(\mathrm{v} / \mathrm{v})$ hydrogen peroxide, followed by two washes $(2 \mathrm{~min})$ in de-ionized water. Photo-bleaching with 5 min under UV lamp and Oil Red O emersion for 2 min were applied to quench auto-fluorescence. Sections were then blocked for $30 \mathrm{~min}$ with Tris-buffered saline (TBS) solution and $3 \%$ bovine serum albumin (w/v). Primary antibodies anti-F4/80 (1:50, Cl:A3-1, MCA497, AbD Serotec), anti-Plin1 (1:100, D1D8, 08/2016 Cell Signalling Technologies) diluted in TBS $+1 \%(\mathrm{w} / \mathrm{v})$ BSA were applied for $2 \mathrm{~h}$ at room temperature, slides were then washed in TBS $+0.1 \%(\mathrm{v} / \mathrm{v})$ Tween20 three times for $5 \mathrm{~min}$, and the secondary antibodies anti-Rat TRITC-conjugated secondary antibody (A18870, ThermoFisher); anti-Rabbit FITC-conjugated secondary antibody (F2765, Life Technologies) were applied for $1 \mathrm{~h}$ at room temperature in the dark. Slides were then washed in TBS + $0.1 \%(\mathrm{v} / \mathrm{v})$ Tween 20 three times for $5 \mathrm{~min}$, slides were mounted with VectaShield hardset mounting media with DAPI, slides were cured overnight at $4^{\circ} \mathrm{C}$ before imaging. Images were acquired on an Axiovert 200M microscope using appropriate filters. Quantitative expression of immunostainings was performed using positive pixels algorithm (Indica Labs) on digital slides (Zeiss). F4/80 staining is expressed as a ratio of Plin1 staining. Quantification method of random fields is an automated observer-independent process based on publicly accessible algorithms. Each biological replicate represented one slide, which was mounted with at least 3 tissue sections, representing 3 technical replicates, the mean of which was presented as the result per biological replicate.

Frozen soleus muscle sections were stained according to standard immunofluorescence protocols. Briefly, transversal sections were brought to room temperature and fixed with $10 \%$ formalin for 10 minutes, followed by permeabilisation through two 5 minute washes with Tris buffered saline (TBS) solution containing $0.5 \%(\mathrm{v} / \mathrm{v})$ Tween 20 . Sections were then blocked with TBS solution containing 3 $\%$ bovine serum albumin. Slides were incubated at $4{ }^{\circ} \mathrm{C}$ in a humidified chamber overnight with anti- 
AIF at a 1:50 dilution in TBS containing 1\% BSA (Sigma SAB3501107) or anti-GLUT4 (Cell Signaling 11/2008) antibodies. Following incubation slides were washed three times for 10 minutes with TBS and incubated in a humidified chamber for 2 hours at room temperature with the following secondary antibodies: AlexaFlour 647 goat-anti-rabbit at 1:200 in TBS containing 1\% BSA (Life technologies A21244) and rabbit-anti-mouse at 1:200 in TBS containing 1\% BSA (abcam ab150127). Slides were washed three times for 5 minutes with TBS. Slides were then mounted with Vectashield Hard-set mounting media containing DAPI. Whole slide scanning was carried out on a Zeiss Axioscan scanner and Zen software. Image quantification was carried out using Visiopharm software applied to whole tissue scans in an observer independent manner following definition of membrane and cytosolic regions. Unless otherwise stated, standard reagents were sourced from Sigma. Quantification was carried out through positive pixel counts of morphologically defined membrane and cytoplasmic regions with the use of Visiopharm software.

\section{Adipose cell isolation and flow cytometry analyses}

The stromal vascular fraction (SVF) containing mononuclear cells and preadipocytes was extracted from adipose tissue. Adipose tissue from mice was digested using $10 \mathrm{~mL}$ digestion solution $(7 \mathrm{~mL}$ Hank's Solution, $3 \mathrm{~mL} 7.5 \%$ BSA and $20 \mathrm{mg}$ collagenase type II, Sigma). The digestion was performed at $37^{\circ} \mathrm{C}$ using a shaker at $100 \mathrm{rpm}$ for $20 \mathrm{~min}$. After digestion, the adipocyte fraction (floating) was isolated and the solution containing the SVF was centrifuged at $1500 \mathrm{rpm}$ at $4^{\circ} \mathrm{C}$ for 5 min. The SVF pellet was resuspended in $1 \mathrm{~mL}$ fluorescence-activated cell sorter (FACS) buffer. After 15 min incubation with Fc Block (2.4G2, BD Biosciences), SVF cells were stained with appropriate antibodies conjugated to fluorochromes or isotype controls for $30 \mathrm{~min}$ at $4^{\circ} \mathrm{C}$ in the dark: $\mathrm{CD} 45$ (30F11), F4/80 (BM8), CD11b (M1/70), CMHII (M5/114.15.2) from eBiosciences, CD11c (HL3) from BD Biosciences and CD206 (C068C2) from Biolegend. Samples were acquired using an Fortessa cytometer (Becton Dickinson) and analyzed with FlowJo (TreeStar) software programs.

\section{Adipose tissue culture}

Mouse adipose tissue biopsies $(0.1 \mathrm{~g})$ were minced and incubated in $1 \mathrm{~mL}$ of endothelial cell basal medium (PromoCell) containing 1\% bovine serum albumin, penicillin $(100 \mathrm{U} / \mathrm{mL})$ and streptomycin $(100 \mathrm{U} / \mathrm{mL})$. Adipose tissue-conditioned medium (ATCM) were recovered after $24 \mathrm{~h}$ and stored at $80^{\circ} \mathrm{C}$ until analysis.

\section{Cytokine quantification}

Cytokine concentrations from ATCM were analyzed using ELISA kits. Adiponectin, IL-17, IL-5 ELISA kits were from R\&D Sytems. IFN- $\gamma$, IL-6, IL-4 and IL-10 ELISA kits were bought from BD. IL-22 ELISA kit was from eBiosciences. IL-17 and IL-22 were measured in PPs (Peyer's patches) or intestine extracts. Briefly, PPs or intestines were lysed in detergent buffer (RIPA) containing protease inhibitor (Roche). After centrifugation $13000 \mathrm{~g}-10 \mathrm{~min}$ at $4^{\circ} \mathrm{C}$, protein quantification was performed on supernatants and then supernatants were stored at $-20^{\circ}$ until ELISA assay. IFN $\gamma$ levels in plasma and WAT were determined using the murine IFN- $\gamma$ ELISA kit from Diaclone SAS (Besançon, France) and according to the manufacturer's instructions.

\section{Quantitative Real time PCR}

Macrophages and intestines were lysed in detergent buffer RLT and then subjected to RNA extraction and reverse transcription (Qiagen). Then, quantitative real-time PCR was performed on an ABI PRISM 7700 (Applied Biosystems) in triplicates. Cycle threshold for Gapdh (primers: Gapdh-R, 5'CGTCCCGTAGACAAAATGGTGAA-3'; Gapdh-L, 5'-GCCGTGAGTGGAGTCATACTGGAACA3') was used to normalize gene expression. Primers for Ido1 are: Ido1-R, 5'ATATATGCGGAGAACGTGGAAAAAC-3', Ido1-L, 5'-CAATCAAAGCAATCCCCACTGTATC3'. Reg3g-R 5'-TTCCTGTCCTCCATGATCAAAA-3' and Reg3g-L 5'- 
CATCCACCTCTGTTGGGTTCA-3'; and Reg3b-R 5'-ATGCTGCTCTCCTGCCTGATG-3' and Reg3b-L 5'-CTAATGCGTGCGGAGGGTATATTC-3. PCR conditions were $10 \mathrm{~min}$ at $95^{\circ} \mathrm{C} ; 35$ cycles of $95^{\circ} \mathrm{C}$ for $15 \mathrm{~s}, 60^{\circ} \mathrm{C}$ for $20 \mathrm{~s}$ and $72^{\circ} \mathrm{C}$ for $20 \mathrm{~s}$ and a final extension at $72^{\circ} \mathrm{C}$ for $20 \mathrm{~s}$.

\section{Western blot analysis}

Tissue samples were lysed in detergent buffer (RIPA, Thermo Scientific) containing protease and phosphatase inhibitor mixture (Roche) and sodium orthovanadate. Proteins were separated on 4-12\% NuPage Tris-Bis gels using NuPage MES-SDS running buffer (Invitrogen) and then were transferred onto nitrocellulose membranes. The filters were probed with antibodies directed against phospho-AKT (Cell Signaling \#4060P), AKT (Cell Signaling \#9272S).

\section{Indirect calorimetric measurements}

Calorimetry exploration was performed at the "Functional and Physiological Exploration" facility (Université Paris-Diderot) as previously described ${ }^{39}$. Briefly, body composition was assessed using an Echo Medical systems EchoMRI 100 (Whole Body Composition Analyser, EchoMRI, Houston, USA). Energy expenditure, oxygen consumption and carbon dioxide production, respiratory exchange ratio, food intake and homecage activity were obtained using calorimetric chambers (Labmaster, TSE Systems GmbH, Bad Homburg, Germany). Activity was recorded using infrared light beam-based locomotion monitoring system. Mice were individually housed, fed with a HFD (SSNIFF, Germany) and acclimated to the chambers and drinking and food holders for $48 \mathrm{hr}$ before experimental measurements. Calorimetric data represent mean of at least $96 \mathrm{hr}$ measurement. Extensive analysis of energy expenditure $(\mathrm{kcal} / \mathrm{hr})$ and $\mathrm{VO} 2$ consumption $(\mathrm{mL} / \mathrm{hr})$ was performed using a regression based approach, analysis of covariance (ANCOVA, Minitab 16, Paris France), as previously described ${ }^{40}$, to take account of the mass effects with body weight, lean mass and fat free mass as covariance. Whole body mass and lean body mass were found to be dependent variables $(P>$ 0.05 from Analysis of covariance). Total energy excretion in feces was estimated by bomb calorimetry (Phenomin facility).

\section{Intestinal content DNA extraction}

Fecal genomic DNA was extracted from the weighted stool samples using a method that was previously described ${ }^{27}$, which is based on the European MetaHIT DNA extraction method.

\section{6s rRNA gene sequencing}

16s rDNA gene sequencing of fecal DNA samples was performed as previously described ${ }^{27}$. Briefly, the V3-V4 region (16S (sense) 5'-TACGGRAGGCAGCAG-3' and (antisense) 5'CTACCNGGGTATCTAAT-3') was amplified and sequencing was done using an Illumina MiSeq platform (GenoScreen, Lille, France). Raw paired-end reads were subjected to the following process: (1) quality-filtering using the PRINSEQ-lite PERL script38 by truncating the bases from the $3^{\prime}$ end that did not exhibit a quality $<30$ based on the Phred algorithm; (2) paired-end read assembly using FLASH (fast length adjustment of short reads to improve genome assemblies) ${ }^{41}$ with a minimum overlap of 30 bases and a 97\% overlap identity; and (3) searching and removing both forward and reverse primer sequences using CutAdapt, with no mismatches allowed in the primers sequences. Assembled sequences for which perfect forward and reverse primers were not found were eliminated. Sequencing data were analyzed using the quantitative insights into microbial ecology (QIIME 1.9.1) software package. The sequences were assigned to OTUs using the UCLUST algorithm ${ }^{42}$ with a $97 \%$ threshold of pairwise identity and classified taxonomically using the Greengenes reference database ${ }^{43}$. Rarefraction was performed ( 8,000 sequences per sample) and used to compare abundance of OTUs across samples. Biodiversity indexes were used to assess alpha diversity and $\alpha$ and $\beta$ diversities were estimated using phylogenetic diversity and unweighted UniFrac. Principal component analyses (PCA) of The Bray Curtis distance with each sample colored according to phenotype were built and used to assess the variation between experimental groups. The LDA effect size algorithm was used to identify taxa that are specific to experimental group ${ }^{44}$. 
The H1L1.1c2 cell line containing a stably integrated dioxin response elements (DRE)-driven firefly luciferase reporter plasmid pGudLuc1.1 has been described previously ${ }^{27}$. The cells were seeded in 96well plates at $7.5 \times 10^{4}$ cells/well in $100 \mu$ of complete Dulbecco's modified Eagle's medium (DMEM) (with $10 \%$ heat-inactivated FCS, $50 \mathrm{IU} / \mathrm{ml}$ penicillin, and $50 \mu \mathrm{g} / \mathrm{ml}$ streptomycin; SigmaAldrich) and cultured $\left(37^{\circ} \mathrm{C}, 10 \% \mathrm{CO}_{2}\right)$ for $24 \mathrm{~h}$ before treatment. This cell line tested negative for mycoplasma contamination and was used in this study to determine AHR activity of small intestine content. To assess agonistic activity, the cells were treated with small intestine content suspensions diluted at 1:10 in complete DMEM. Controls consisted of cells treated with DMEM as the negative control, or FICZ (Sigma) diluted in DMEM as the positive control. After $24 \mathrm{~h}$ of incubation, wells were washed with $100 \mu \mathrm{l}$ PBS, and $50 \mu \mathrm{l}$ Promega lysis buffer was added to each well. The plates were shaken for $30 \mathrm{~min}$ to lyse the cells. After adding $100 \mu \mathrm{l}$ of luciferase reagent (Promega), luciferase activity was measured using a luminometer. The results were normalized based on the negative luciferase activity of the control.

\section{HPLC quantifications}

Thawed stools from mice were extracted as previously described ${ }^{45}$. L-tryptophan (Trp) and Lkynurenine (Kyn) were measured via HPLC using a coulometric electrode array (ESA Coultronics, ESA Laboratories, Chelsford,MA, USA) ${ }^{46}$. Quantifications were performed by referencing calibration curves obtained with internal standards. Other compounds (IAA) were quantified via liquid chromatography coupled to mass spectrometry (LC-MS) by using a Waters ACQUITY ultraperformance liquid chromatography (UPLC) system equipped with a binary solvent delivery manager and sample manager (Waters Corporation, Milford, MA, USA) and that was coupled to a tandem quadrupole-time-of-flight (Q-TOF) mass spectrometer equipped with an electrospray interface (Waters Corporation). Compounds were identified by comparing with the accurate mass and the retention time of reference standards in our in-house library, and the accurate masses of the compounds were obtained from web-based resources, such as the Human Metabolome Database (http://www.hmdb.ca) and the METLIN database (http://metlin.scripps.edu).

\section{ATP measurements}

The firefly luciferase bioluminometric assay was used to measure the muscular ATP content as previously described ${ }^{47}$.

\section{2-deoxy-2-[18F]fluoro-D-glucose ([18F]FDG) PET-CT Imaging}

Mice were anesthetized with isoflurane (IsoVet 100\%; Centravet, France) in 100\% $\mathrm{O}_{2}(4 \%$ isoflurane for induction; $1-2 \%$ for maintenance). Mice were weighted, and placed on a heated plate (Minerve, France). A customized catheter with a $26 \mathrm{G}$ needle (Fischer Scientific, France) connected to a 5-cm polyethylene tubing (Tygon Microbore Tubing, 0.010" x 0.030"OD; Fisher Scientific, France) was installed in the lateral tail vein of the mice. $10 \mathrm{MBq}$ of 2-deoxy-2- $\left[{ }^{18} \mathrm{~F}\right]$ fluoro-D-glucose $\left(\left[{ }^{18} \mathrm{~F}\right] \mathrm{FDG}\right.$; Gluscan, Advanced Applied Applications, France) in $200 \mu 1$ of saline solution were injected in the mice. The mice were then put back in their cages and left awake for $30 \mathrm{~min}$. Mice were anesthetized again and installed in the imaging bed of the PET/CT scan (nanoScan, Mediso, Hungary). CT scans were performed first using the following parameters: mode semi-circular, tension of $39 \mathrm{kV}, 720$ projections full scan, $300 \mathrm{~ms}$ per projection, binning 1:4. List-mode PET data were collected between 45 and 60 min post injection of $\left[{ }^{18} \mathrm{~F}\right] \mathrm{FDG}$, binned using a 5-ns time window, a 400 - to $600-\mathrm{keV}$ energy window, and a 1:5 coincidence mode. In vivo PET acquisitions were reconstructed using the TeraTomo reconstruction engine (3D-OSEM based manufactured customized algorithm) with expectation maximization iterations, scatter and attenuation corrections. Images were analyzed using the software PMOD (PMOD Technologies LLC). Standardized Volume of Interest (VOI) was drawn in each organ 
of interest and Standardized Uptake Values (SUV) were calculated by dividing the mean tissue radioactivity concentration by the injected dose and body weight.

\section{NanoString.}

NanoString analysis was performed and analyzed according to the manufacturer's recommendations.

\section{Human population}

Feces from healthy non obese individuals were recruited in the Gastroenterology Department of the Saint Antoine Hospital (Paris, France) and provided informed consent. Plasmas were from healthy non obese individuals $(n=20)$ from EFS, HEGP hospital (Paris, France).

49 women with morbid obesity were followed at the Endocrinology Service of the Hospital Universitari de Girona Dr Josep Trueta (Girona, Spain, $n=25$ ) and at the Center for Atherosclerosis of Policlinico Tor Vergata University of Rome (Rome, Italy, $n=24)$. Pre-established inclusion criteria: all subjects were of Caucasian origin; the subjects reported a stable body weight 3 months preceding the study, were free of any infections 1 month before and had no systemic disease. Pre-established exclusion criteria: subjects with liver disease, specifically HCV infection and tumor disease, and subjects with thyroid dysfunction were excluded by biochemical workup. All subjects gave written informed consent, validated and approved by the ethical committee of the Hospital Universitari Dr Josep Trueta (Comitè d'Ètica d'Investigació Clínica) and Policlinico Tor 388 Vergata University of Rome (Comitato Etico Indipendente).

\section{Subjects with type 2 diabetes}

We recruited subjects with treatment-naive type 2 diabetes (T2D), as recently reported ${ }^{48}$. Inclusion criteria were: (i) aged between 18 and 65 years; (ii) T2D diagnosis in the previous 6 months, as defined by the American Diabetes Association Criteria; (iii) absence of systemic and metabolic disease other than T2D, and absence of infection within the previous month; (iv) absence of diet or medication that might interfere with glucose homeostasis, such as glucocorticoids or antibiotics in the previous 3 months; and (v) HbAlc lower than 9\%. Exclusion criteria were: (i) clinically significant major systemic disease, including malignancy; (ii) clinical evidence of hemoglobinopathies or anemia; (iii) history of drug or alcohol abuse, defined as $>80 \mathrm{~g} / \mathrm{d}$ in men and $>40 \mathrm{~g} / \mathrm{d}$ in women; (iv) acute major cardiovascular event in the previous 6 months; (v) acute illnesses or current evidence of acute or chronic inflammatory or infective disease; and (vi) mental illness rendering the participants unable to understand the nature, scope, and possible consequences of the study.

All individuals gave written informed consent. The experimental protocol was approved by the Ethics Committee and the Committee for Clinical Investigation of the Hospital Universitari Dr. Josep Trueta (Girona, Spain). We certify that all applicable institutional regulations concerning the ethical use of information and samples from human volunteers were followed during this research. Complete clinical trial registration is deposited in the EU clinical trials register (EudraCT number 2010-022394-34). We report here the findings in the baseline samples from these subjects before entering the trial.

\section{Analytical determinations (human)}

Total plasma cholesterol was measured by an enzymatic, colorimetric method through the cholesterol esterase/cholesterol oxidase/peroxidase reaction (Cobas CHOL2). HDL cholesterol was quantified by a homogeneous enzymatic colorimetric assay through the cholesterol esterase/cholesterol oxidase/peroxidase reaction (Cobas HDLC3). Total plasma triglycerides were measured by an enzymatic, colorimetric method with glycerol phosphate oxidase and peroxidase (Cobas TRIGL). Serum aspartate transaminase (AST) was measured by colorimetry using automated tests (Roche Diagnostics $\mathrm{GmbH}$, Mannheim, Germany). Intra- and inter-assay coefficients of variation were $<4 \%$.

\section{Euglycemic hyperinsulinemic clamp (human)}


Insulin action was determined by the euglycemic hyperinsulinemic clamp. After an overnight fast, two catheters were inserted into an antecubital vein, one for each arm, used to administer constant infusions of glucose and insulin, and to obtain arterialized venous blood samples. A 2-h euglycemic hyperinsulinemic clamp was initiated by a two step primed infusion of insulin $\left(80 \mathrm{mU} / \mathrm{m}^{2} / \mathrm{min}\right.$ for 5 $\mathrm{min}, 60 \mathrm{mU} / \mathrm{m}^{2} / \mathrm{min}$ for $5 \mathrm{~min}$ ) immediately followed by a continuous infusion of insulin at a rate of $40 \mathrm{mU} / \mathrm{m}^{2} / \mathrm{min}$ (regular insulin; Actrapid, Novo Nordisk, NJ). Glucose infusion began at minute 4 at an initial perfusion rate of $2 \mathrm{mg} / \mathrm{kg} / \mathrm{min}$, then was adjusted to maintain plasma glucose concentration at 4.9-5.5 $\mathrm{mmol} / \mathrm{L}$. Blood samples were collected every $5 \mathrm{~min}$ for determination of plasma glucose and insulin. Insulin sensitivity was assessed as the mean glucose infusion rate during the last $40 \mathrm{~min}$. In the stationary equilibrium, the amount of glucose administered (M) equals the glucose taken by the body tissues and is a measure of overall insulin sensitivity.

\section{Statistical analysis.}

Values are expressed as means \pm s.e.m. The differences between groups were assessed using nonparametric Mann-Whitney test or an unpaired Student's $t$-test (two-sided). Values were considered significant at $P<0.05$. For human data, given the sample size, non-parametric Mann-Whitney test was used to assess the differences between 2 groups or Kruskal-Wallis for multiple comparisons. Spearman's rank correlation coefficient $(r)$ was used to analyze the correlations between Kyn and metabolic and clinical parameters in subjects with obesity. Differences corresponding to $P<0.05$ were considered significant. Statistical analysis was performed with GraphPad Prism (San Diego, CA, USA).

\section{Life Sciences Reporting Summary.}

Further information on experimental design is available in the Life Sciences Reporting Summary.

\section{Data availability}

The data for the findings of this study are available from the corresponding author upon reasonable request. European Nucleotide Archive: the sequencing data are deposited under accession number PRJEB25438.

\section{References}

35. Sonnenberg, G.F. \& Artis, D. Innate lymphoid cell interactions with microbiota: implications for intestinal health and disease. Immunity 37, 601-610 (2012).

36. Berglund, E.D., et al. Glucose metabolism in vivo in four commonly used inbred mouse strains. Diabetes 57, 1790-1799 (2008).

37. Allison, D.B., Paultre, F., Maggio, C., Mezzitis, N. \& Pi-Sunyer, F.X. The use of areas under curves in diabetes research. Diabetes care 18, 245-250 (1995).

38. Ferchaud-Roucher, V., Pouteau, E., Piloquet, H., Zair, Y. \& Krempf, M. Colonic fermentation from lactulose inhibits lipolysis in overweight subjects. American journal of physiology. Endocrinology and metabolism 289, E716-720 (2005).

39. Cansell, C., et al. Dietary triglycerides act on mesolimbic structures to regulate the rewarding and motivational aspects of feeding. Mol Psychiatry 19, 1095-1105 (2014).

40. Speakman, J.R., Fletcher, Q. \& Vaanholt, L. The '39 steps': an algorithm for performing statistical analysis of data on energy intake and expenditure. Disease models \& mechanisms 6 , 293-301 (2013).

41. Schmieder, R. \& Edwards, R. Quality control and preprocessing of metagenomic datasets. Bioinformatics 27, 863-864 (2011).

42. Edgar, R.C. Search and clustering orders of magnitude faster than BLAST. Bioinformatics 26, 2460-2461 (2010). 
43. McDonald, D., et al. An improved Greengenes taxonomy with explicit ranks for ecological and evolutionary analyses of bacteria and archaea. The ISME journal 6, 610-618 (2012).

44. Segata, N., et al. Metagenomic biomarker discovery and explanation. Genome biology 12, R60 (2011).

45. Gao, X., et al. Metabolite analysis of human fecal water by gas chromatography/mass spectrometry with ethyl chloroformate derivatization. Analytical biochemistry 393, 163-175 (2009).

46. Maneglier, B., et al. Simultaneous measurement of kynurenine and tryptophan in human plasma and supernatants of cultured human cells by HPLC with coulometric detection. Clin Chem 50, 2166-2168 (2004).

47. Ferraresi, C., et al. Time response of increases in ATP and muscle resistance to fatigue after low-level laser (light) therapy (LLLT) in mice. Lasers in medical science 30, 1259-1267 (2015).

48. Wu, H., et al. Metformin alters the gut microbiome of individuals with treatment-naive type 2 diabetes, contributing to the therapeutic effects of the drug. Nat Med 23, 850-858 (2017). 
Figure 1

a

b

C

d
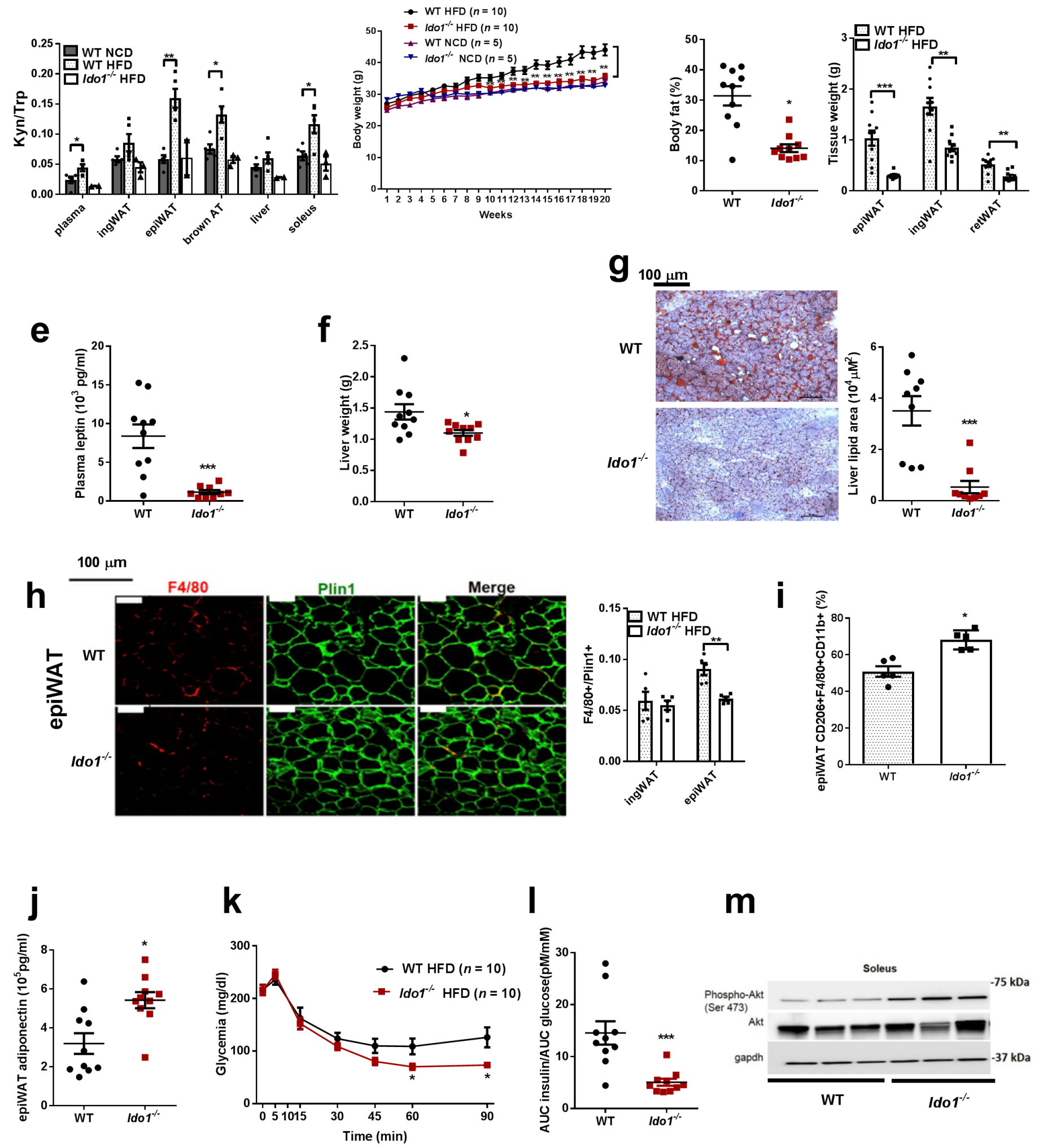

\section{m}

n
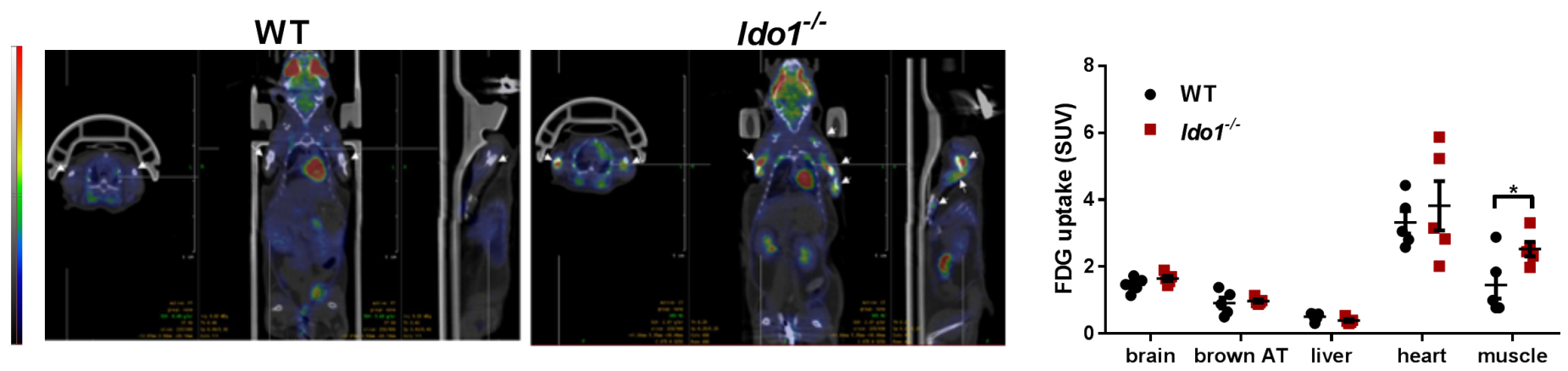


\section{Figure 2}

a
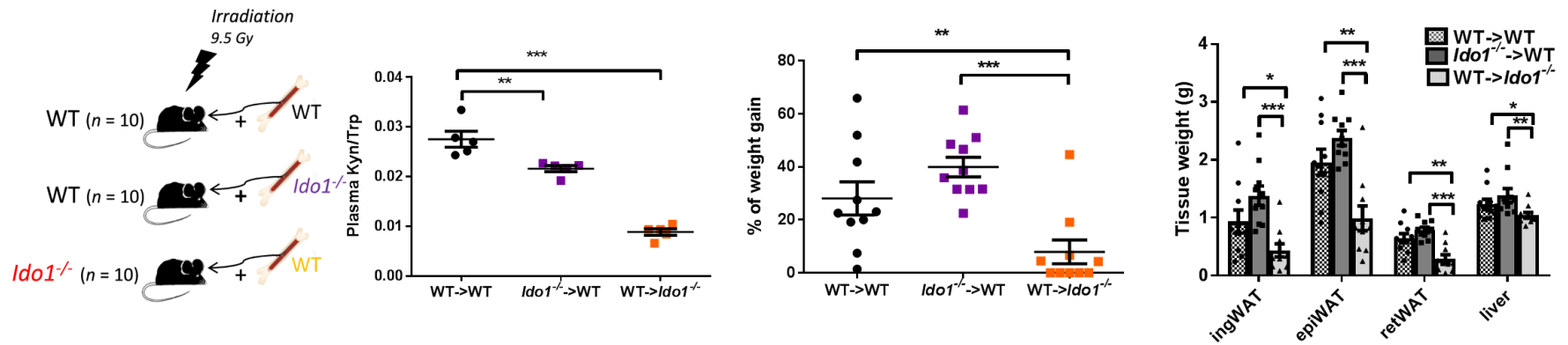

d

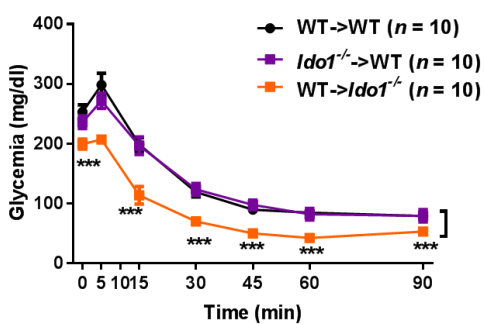

g

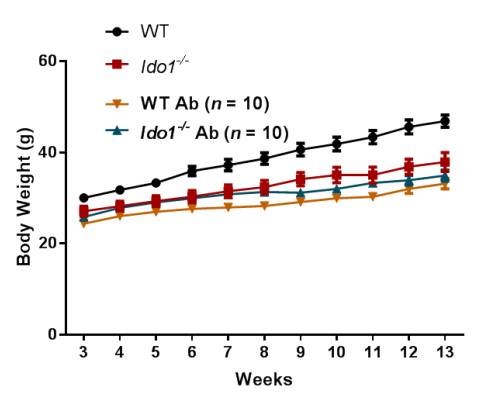

j
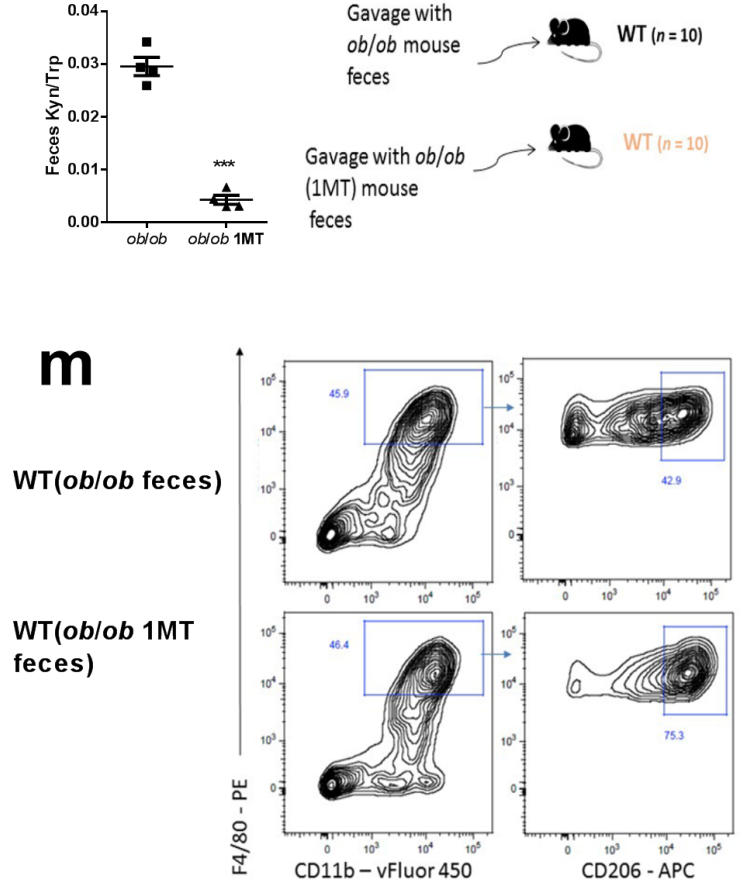

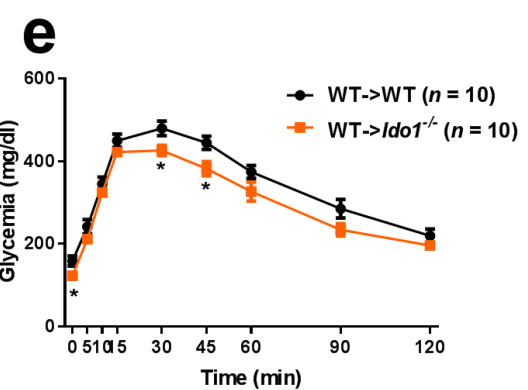

h

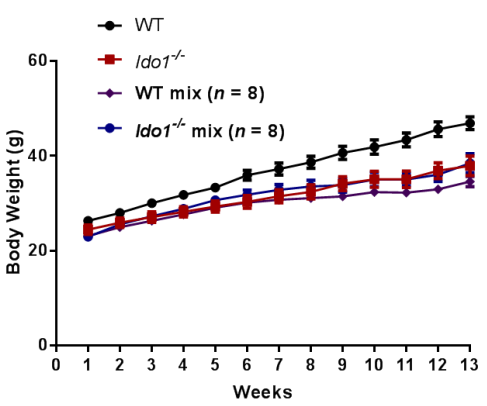

k

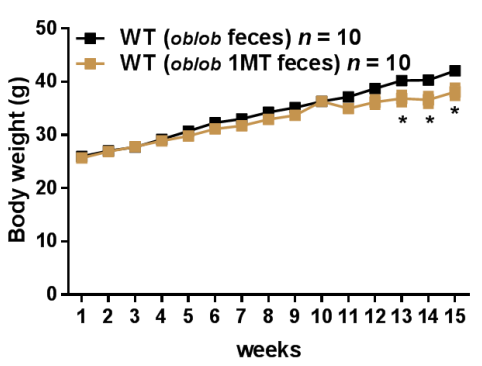

7

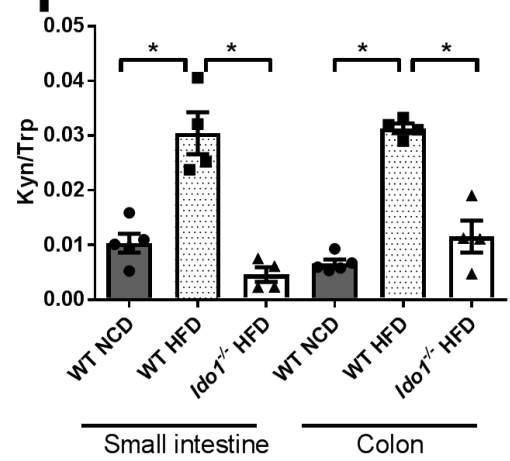

i

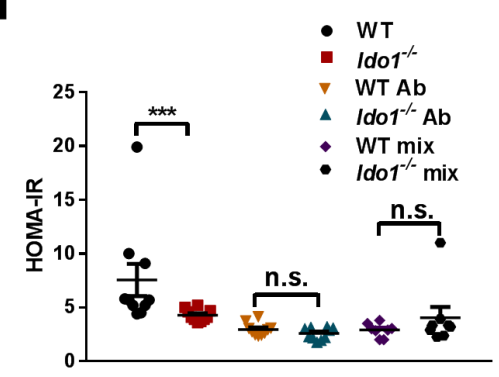

I

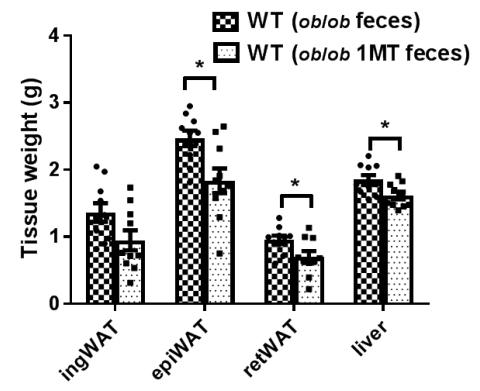

n

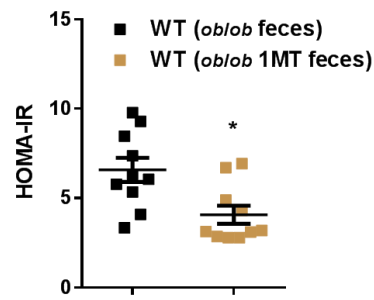




\section{Figure 3}

a

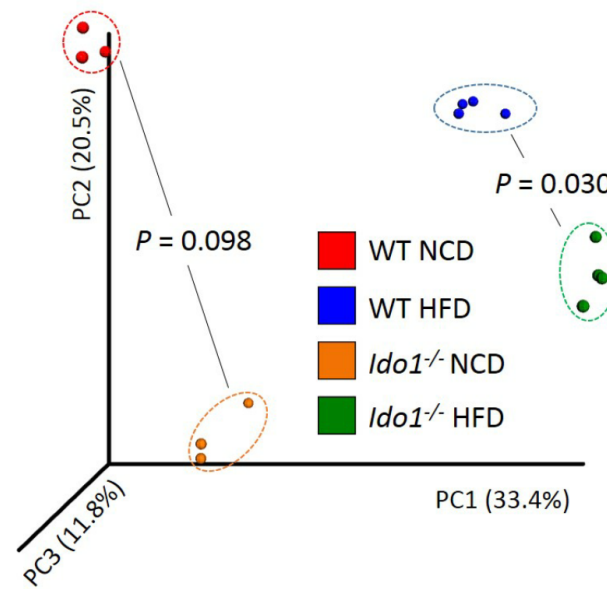

b

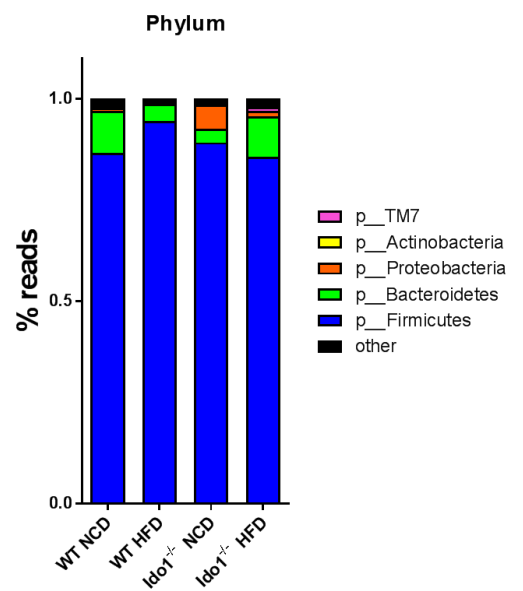

C

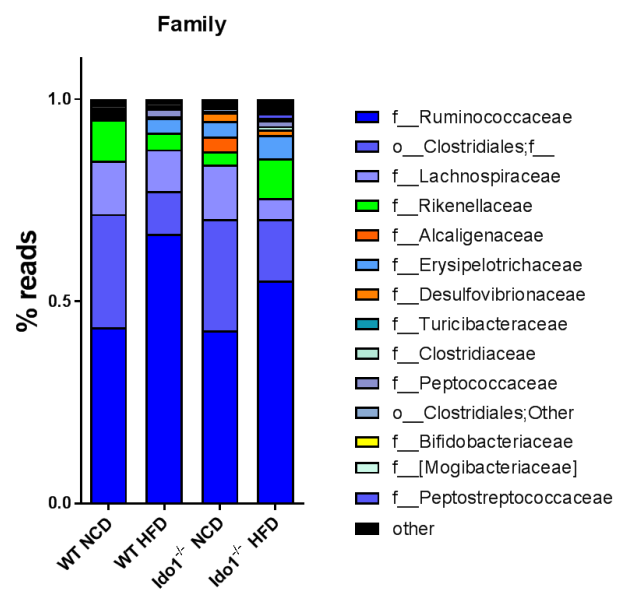

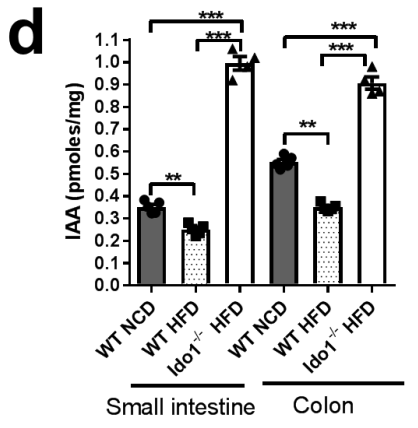

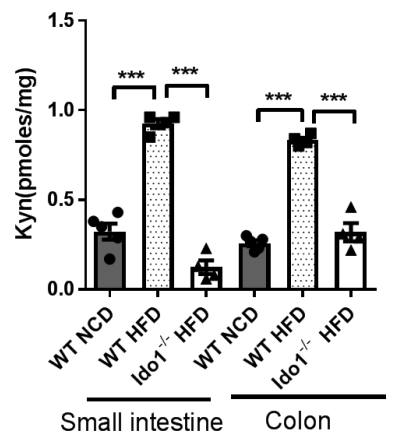

e

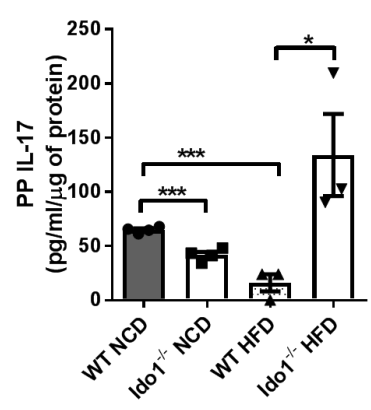

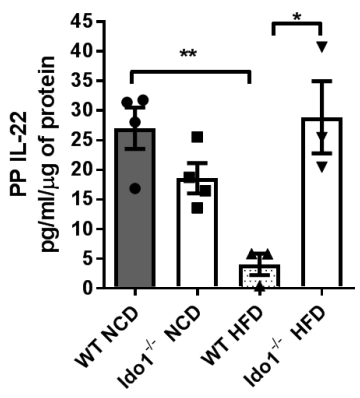
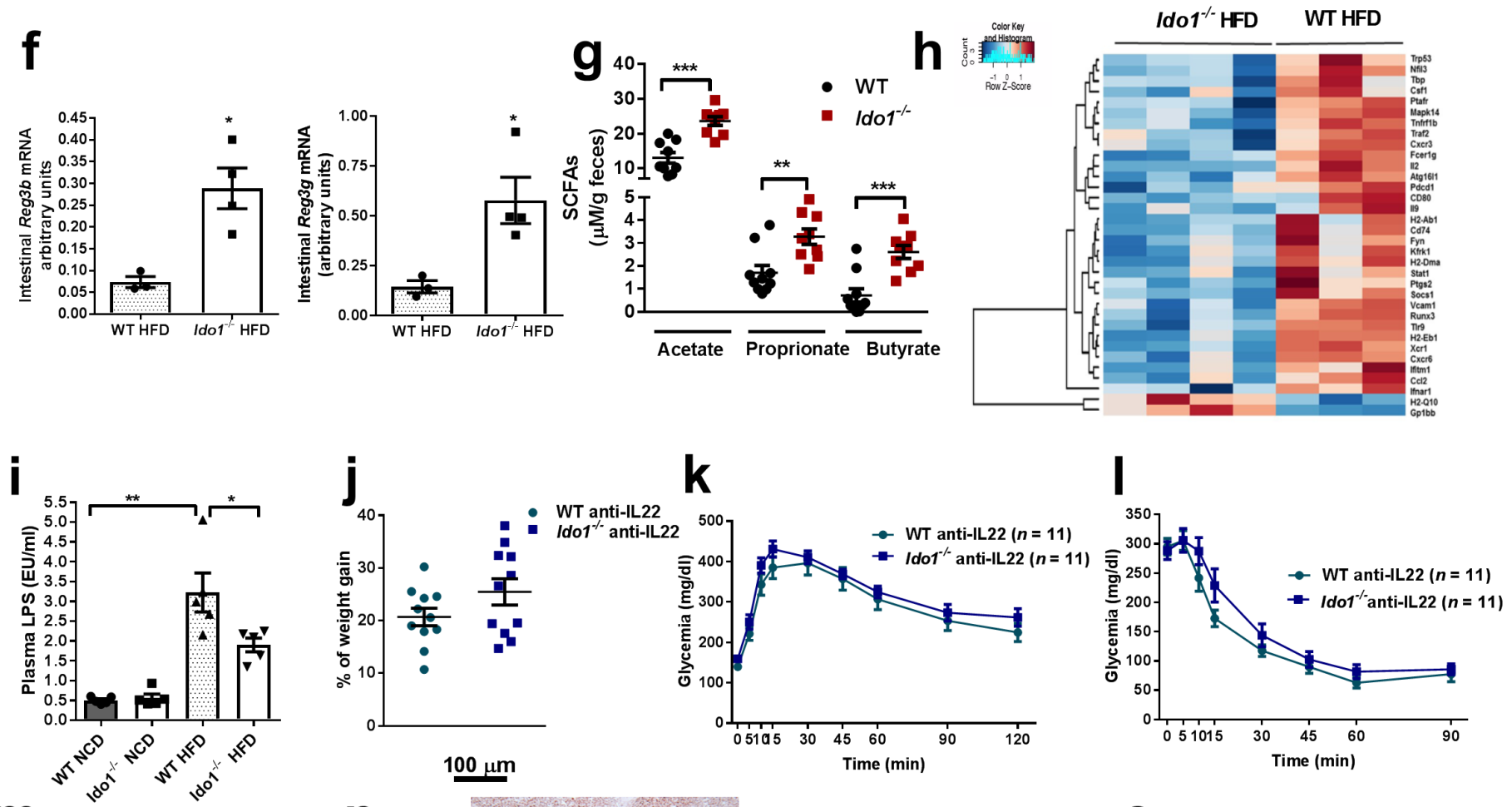

m

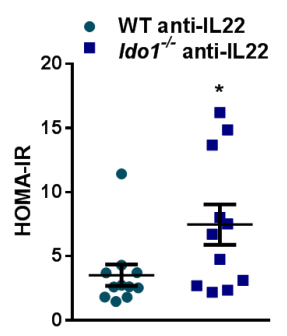

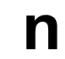

WT anti-IL22

Ido ${ }^{-/}$anti-IL22

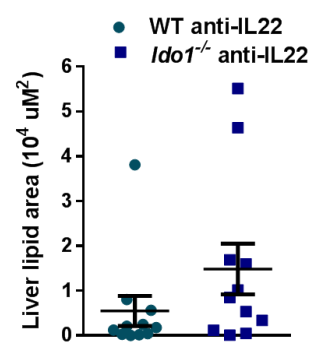

0

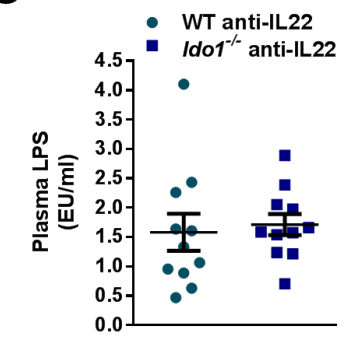


Figure 4
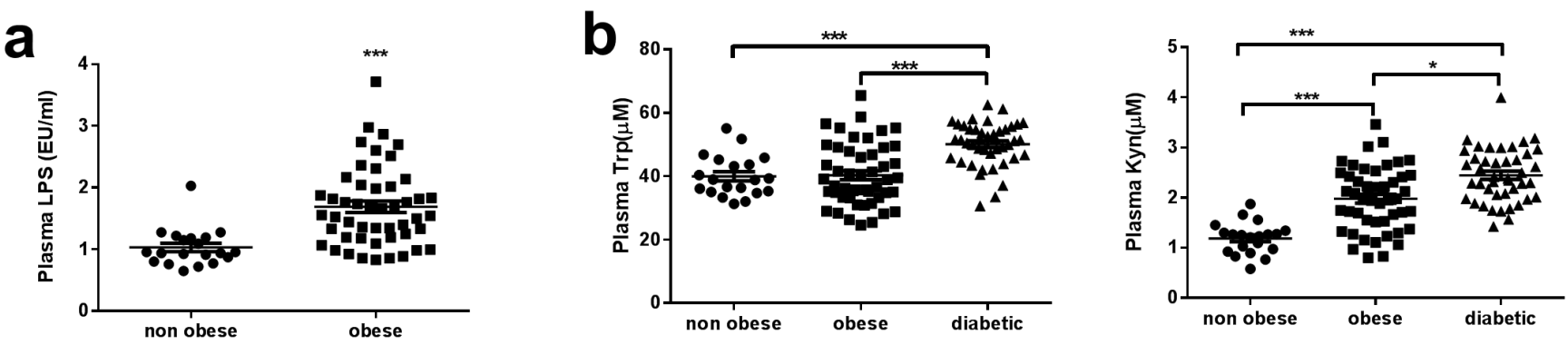

C
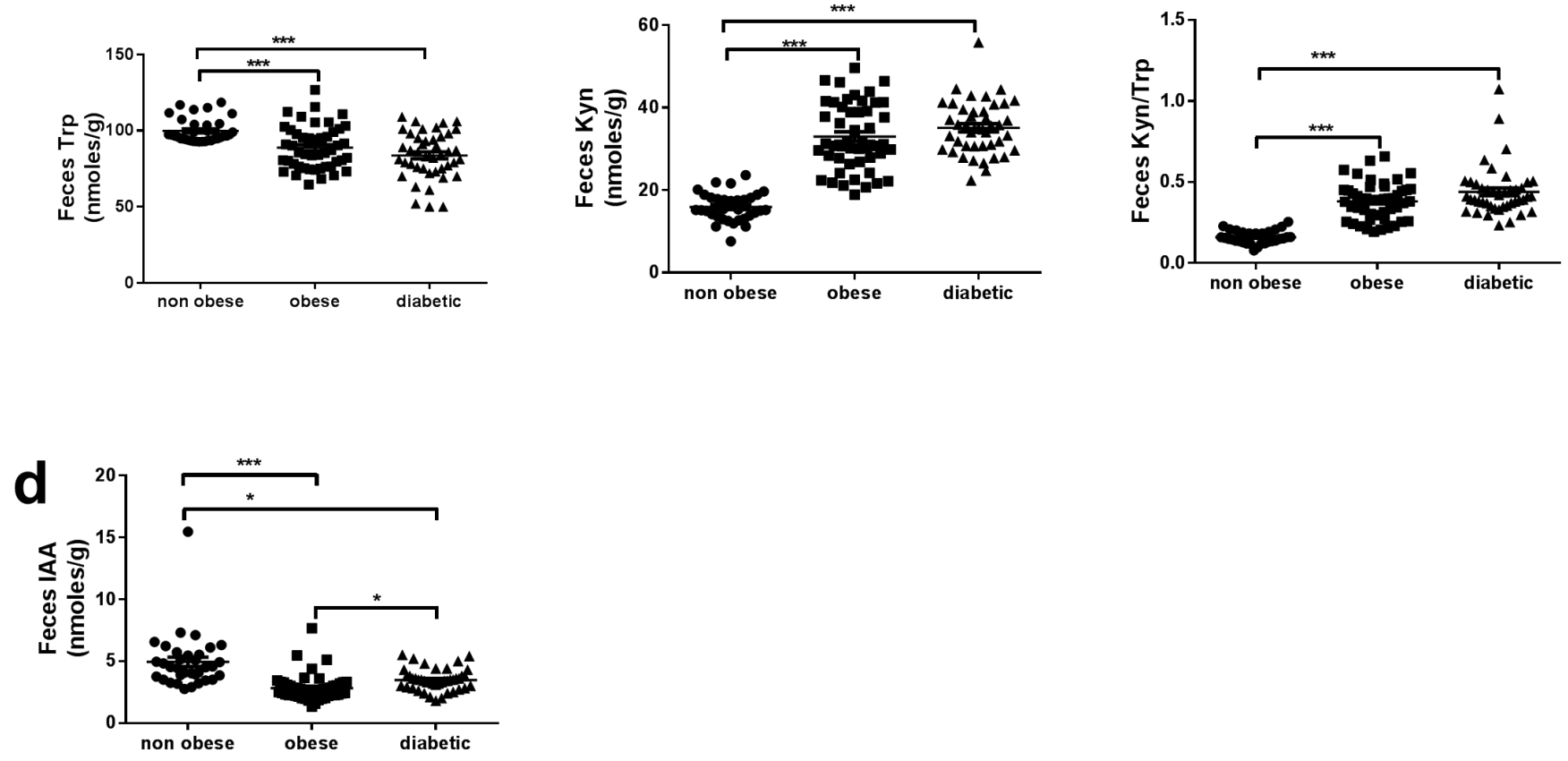
Supplementary Table Clinical characteristics of the subjects

\begin{tabular}{|c|c|c|c|c|}
\hline & Obese & Diabetic & Controls 1 (feces) & Controls 2 (plasma) \\
\hline Number & 49 & 43 & 34 & 20 \\
\hline Sex Ratio (f/m) & $39 / 10$ & $24 / 19$ & $20 / 14$ & $15 / 5$ \\
\hline Age (years) & $43.29 \pm 1.53$ & $52.28 \pm 1.38$ & $35.08 \pm 2.26$ & $37.35 \pm 3.36$ \\
\hline Weight (kg) & $128.60 \pm 3.98$ & $96.70 \pm 3.64$ & $64.79 \pm 1.74$ & $69.05 \pm 3.20$ \\
\hline BMI (kg/m2) & $47.29 \pm 1.19$ & $36.42 \pm 1.22$ & $22.65 \pm 0.43$ & $23.80 \pm 0.79$ \\
\hline Waist (cm) & $135.2 \pm 3.78$ & $112.42 \pm 2.5$ & & \\
\hline Fat mass $(\%)$ & $57.99 \pm 2.02$ & $36.91 \pm 3.42$ & & \\
\hline $\operatorname{AST}(\mathbf{U} / \mathbf{L})$ & $24.59 \pm 1.68$ & $41.47 \pm 6.5$ & & \\
\hline AUC glucose $\mathbf{O G T T}(\mathbf{m m o l} / \mathrm{L} / 120 \mathrm{~min})$ & $36.46 \pm 3.43$ & & & \\
\hline GDR (mg/kg/min) & $3.09 \pm 0.34$ & & & \\
\hline Total cholesterol (mmol/l) & $187.8 \pm 4.08$ & $203.56 \pm 5.22$ & & \\
\hline HDL-cholesterol (mmol/l) & $45.75 \pm 1.58$ & $45.77 \pm 1.70$ & & \\
\hline Triglycerides ( mmol/l) & $125.30 \pm 6.62$ & $143.53 \pm 13.40$ & & \\
\hline TG/HDL-chol & $3.04 \pm 0.24$ & $3.59 \pm 0.44$ & & \\
\hline LPS (EU/ml) & $1.68 \pm 0.09$ & & & $1.03 \pm 0.06$ \\
\hline Plasma Trp $(\mu \mathrm{M})$ & $40.35 \pm 1.39$ & $50.09 \pm 1.05$ & & $39.99 \pm 1.45$ \\
\hline Plasma Kyn $(\mu M)$ & $1.97 \pm 0.08$ & $2.44 \pm 0.08$ & & $1.18 \pm 0.07$ \\
\hline Feces Trp (nmol/g) & $88.83 \pm 1.98$ & $83.74 \pm 2.32$ & $99.98 \pm 1.34$ & \\
\hline Feces Kyn (nmol/g) & $32.97 \pm 1.16$ & $35.09 \pm 0.98$ & $15.88 \pm 0.60$ & \\
\hline Feces IAA (nmol/g) & $2.83 \pm 0.15$ & $3.47 \pm 0.13$ & $4.94 \pm 0.37$ & \\
\hline
\end{tabular}

Values are mean \pm sem

BMI, body mass index; AUC ${ }^{\text {glucose }}$ OGTT: area under the curve of glucose during an oral glucose tolerance test; GDR (glucose disposal rate) glucose infusion during euglycemic hyperinsulinemic clamp, AST aspartate aminotransferase, TG triglycerides, Trp tryptophan, Kyn kynurenine, LPS lipopolysaccharide, IAA Indole-3acetic acid. 


\section{Supplemental Figure Legends}

Supplementary Fig. 1 Idol mRNA is induced by the high fat diet (HFD). (a) IdoI mRNA in epiWAT, ingWAT and soleus muscle in WT and $I d o 1^{-/}$mice fed with either NCD or HFD during 20 weeks $(n=$ 5 per group). (b) IFN- $\gamma$ levels in epiWAT and plasma of WT mice on either NCD or HFD during 20 weeks ( $n=5$ per group). Data are expressed as mean \pm sem. $* * P<0.001, * * * P<0.0001$.

Supplementary Fig. 2 IDO deficiency has no major effects in basal conditions. (a-d) Weights of epiWAT, ingWAT, retWAT and liver (a), OGTT(b), ITT (c), and HOMA-IR (d), in WT and Idol ${ }^{-/}$ mice fed with NCD during 20 weeks ( $n=5$ per group). Data are expressed as mean \pm sem. $* P<0.05$.

Supplementary Fig. $3 \mathrm{Idol}^{-/-}$mice fed with HFD are protected against obesity and its complications. (a-c) Lean mass measured by Echo-MRI (a), representative pictures (left) and quantification of CD68 (right) staining surface in livers of WT and $I d o I^{-/-}$mice fed with HFD during 20 weeks $(n=9$ per group)(b), cytokine production by ingWAT explants from WT and $I d o 1^{-l_{-}}$mice fed with HFD $(n=5$ per group) (c). Scale bars $100 \mu \mathrm{M}$. (d) Plasma insulin concentrations during OGTT in WT and $I_{d o I^{-/}}$ mice fed with either NCD ( $n=5$ per group) or HFD ( $n=10$ per group) during 20 weeks. (e) Insulin signaling (pAKT-S473) in liver, ingWAT and epiWAT after $15 \mathrm{~min}$ of $5 \mathrm{U} / \mathrm{kg}$ insulin injection in WT and $I d o I^{-/-}$mice fed with HFD. (f) Representative Scatter plots from the analysis of covariance showing energy expenditure $(\mathrm{kcal} / \mathrm{hr})$ plotted against body weight of HFD-fed WT $(n=11)$ and HFDfed $I d o 1^{-/-}$mice $(n=12)$ with a significant genotype effect $(P<0.05)$ and a significant body weight effect $(P<0.01)$. (g) Food intake normalized on body weight ( $\mathrm{g} / \mathrm{kg}$ body weight $/ \mathrm{hr} ; n=5-6$ per group) expressed as mean of 4 daylights and 4 nights. (h) Energy excretion measured by calorimetric bomb in feces of WT and $I d o 1^{-1-}$ mice fed with HFD ( $n=4$ per group). Data are expressed as mean \pm sem. $* P<$ $0.05, * * P<0.001, * * * P<0.0001$.

Supplementary Fig. $4 \mathrm{Idol}^{-1-}$ mice fed with HFD have a high muscular metabolism. (a, b) Representative pictures and quantifications of staining for respectively glucose transporter 4 (GLUT4) (a), and for apoptosis inducing factor (AIF) as a mitochondrial marker (b), on transverse sections of soleus muscle from $I d o I^{-1-}$ mice $(n=6)$ and WT mice fed with HFD $(n=5)$. Nuclei were counterstained with DAPI. (c) ATP concentrations in soleus muscle of WT $(n=5)$ and $I d o 1^{-/-}$mice $(n$ $=3$ ) fed with HFD. Data are expressed as mean \pm sem. $* P<0.05$, $* * P<0.001$.

Supplementary Fig. 5 IDO inhibition protects against insulin resistance in obesity. (a-e) Weight curves of HFD-fed WT mice treated or not with 1MT diluted in drinking water $(2 \mathrm{mg} / \mathrm{ml})$ during 19 weeks ( $n=10$ per group) (a), plasma IDO activity (Kyn/Trp) (b), production of adiponectin by epiWAT explants ( $n=5$ per group) (c), ITT (d) and HOMA-IR (e), in HFD-fed WT mice treated or not with 1MT ( $n=7$ per group). (f, g) ITT and OGTT in ob/ob mice fed with NCD and treated or not with $1 \mathrm{MT}$ during 14 weeks ( $n=10$ per group). Data are expressed as mean $\pm \operatorname{sem}$. $* P<0.05$, $* * P<$ $0.001, * * * P<0.0001$.

Supplementary Fig. 6 IDO expressed in macrophages has no effects in obesity. (a) Idol mRNA in peritoneal macrophages (pMac) isolated from $I d o I^{\text {flox/flox }}$ mice and $I d o I^{\text {flox/flox }}$ LysM-cre mice after 20 weeks of HFD. (b) Weight curves, (c) weights of epiWAT, ingWAT and retWAT, (d, e) ITT and HOMA-IR in $I d o I^{\text {flox/flox }}$ mice or $I d o I^{\text {flox/lox }}$ LysM-cre mice ( $n=5$ per group) after 20 weeks of HFD. Data are expressed as mean \pm sem. $* * * P<0.0001$.

Supplementary Fig. 7 IDO inhibition and invalidation effects on gut microbiota. (a) PCA plot based on bacterial 16S rDNA gene sequence abundance in fecal content of $o b / o b$ mice treated or not with 1MT. Axes correspond to principal components 1(x-axis), 2 (y-axis) and 3 (z-axis). (b) Bacterial diversity on the basis of shannon in the fecal samples from WT and $I d o I^{-1-}$ mice fed with either NCD or HFD and $o b / o b$ mice treated or not with 1MT. (c) Bacterial taxons differentially expressed in feces from WT or $I d o 1^{-1-}$ mice fed with either NCD or HFD. (d) Bacterial-taxon-based analysis at the phylum level in feces of $o b / o b$ mice treated or not with 1MT. (e) A correlation between presence of 
Clostridiales Lachnospiraceae in feces and plasma LPS. (f) Bacterial taxa differentially enriched in HFD-fed WT and $I d o I^{-/-}$mice (generated using LeFSE analysis). Data are expressed as mean \pm sem. $* * P<0.001$.

Supplementary Fig. 8 Kynurenine has no effects in metabolic syndrome. (a, b) Plasma Kyn (a) and Trp (b) in $I d o 1^{-1-}$ mice supplemented or not with Kyn diluted in drinking water $(2 \mathrm{mg} / \mathrm{ml})$ during 15 weeks of HFD ( $n=10$ per group). (c-d) weight curves (c), and weights of ingWAT, epiWAT, retWAT and liver (d). (e, f) ITT and HOMA-IR in WT, IdoI ${ }^{-/-}$and Kyn-supplemented IdoI ${ }^{-/-}$mice. Data of HOMA-IR are presented as box-and-whisker plots, with the midline representing the median and the whiskers representing maximum and minimum values. Data are expressed as mean \pm sem. $* * * P<$ 0.0001 .

Supplementary Fig. 9 Trp and Trp-derived metabolites in gastrointestinal tract. (a) Schematic representation of the use of Trp in gastrointestinal tract. (b-c) Kyn and IAA levels in small intestines and colons of $o b / o b$ mice treated or not with 1MT ( $n=4$ per group). (d) Trp levels in small intestines and colons from WT mice fed with either NCD or HFD and HFD-fed $I d o 1^{-/-}$mice ( $n=4-5$ per group). (e) Trp levels in small intestines and colons of $o b / o b$ mice treated or not with 1MT during 15 weeks $(n$ $=4$ per group). (f) IAA levels in small intestines of WT mice which received feces from either 1MTtreated or not treated $o b / o b$ mice ( $n=10$ per group). (g) Quantification of AHR activity in fecal samples recovered from WT and $I d o 1^{-1-}$ small intestines ( $n=5$ per group) after 7 weeks of HFD. Data are expressed as mean \pm sem. $* P<0.05, * * * P<0.0001$.

Supplementary Fig. 10 IAA decreases insulin resistance and adipose inflammation. (a-b) HOMA-IR (a), representative cytometry (left) and quantification (right) of M2-like macrophages $(\mathrm{F} 4 / 80+\mathrm{CD} 11 \mathrm{~b}+\mathrm{CD} 206+$ in epiWAT) $(n=5$ per group) (b), from WT mice treated with either the vehicle (DMSO) $(n=8)$ or FICZ (6-formylindolo[3,2-b]carbazole) $(n=7)$ during 15 weeks of HFD. (c-f) Feces IAA levels (c), intestinal IL-22 concentrations (d), ITT (e), and representative cytometry and quantification of CD45+ cells and M2-like macrophages (F4/80+CD11b+CD206+ in epiWAT) ( $n$ $=5$ per group) (f), in WT mice supplemented or not with IAA (in drinking water) and put on HFD during 11 weeks ( $n=9-10$ per group). (g) IL-12 levels in PP of WT mice treated or not with 1MT during 7 weeks of HFD ( $n=5$ per group). (h) IL-22 levels in small intestines from WT mice which received feces from 1MT-treated or untreated $o b / o b$ mice after 15 weeks of HFD. (i-j) Correlations between intestinal IL-22 levels and intestinal IAA (i), or HOMA-IR (j), in WT which received feces from 1MT-treated or untreated $o b / o b$ mice. Data are expressed as mean \pm sem. $* P \leq 0.05$, $* * P<$ $0.001, * * * P<0.0001$.

Supplementary Fig. 11 IL22 neutralisation abrogates protective effects of IDO deficiency against obesity and its complications. (a-b) Body fat $\%$ ( $n=6$ per group) (a), weights of epiWAT, ingWAT and retWAT from WT and $I d o 1^{-1-}$ mice fed with HFD during 12 weeks and treated with mouse neutralizing anti-IL22 antibody (b) ( $n=11$ per group). (c) Representative cytometry (left) and quantification (right) of M2-like macrophages (F4/80+CD11b+CD206+ in epiWAT) ( $n=5$ per group) from WT and $I d o I^{-1-}$ mice fed with HFD during 12 weeks and treated with neutralizing anti-IL22 antibody. (d-h) \% of body fat (d), weights of epiWAT, ingWAT and retWAT (e), ITT (f),OGTT (g) and plasma LPS (h) in WT $(n=5)$ and $\operatorname{Idol}^{-l_{-}}(n=4)$ mice treated with control IgG1 three times per week during 12 weeks of HFD. (d, e and $\mathbf{h}$ ) Data are presented as box-and-whisker plots, with the midline representing the median and the whiskers representing maximum and minimum values. Data are expressed as mean \pm sem. $* P<0.05, * * P<0.001$

Supplementary Fig. 12 Full blot scans of P-AKT, AKT and GAPDH. The blots shown in Fig. 1m are within squares. 


\section{Supplementary Fig 1}

a
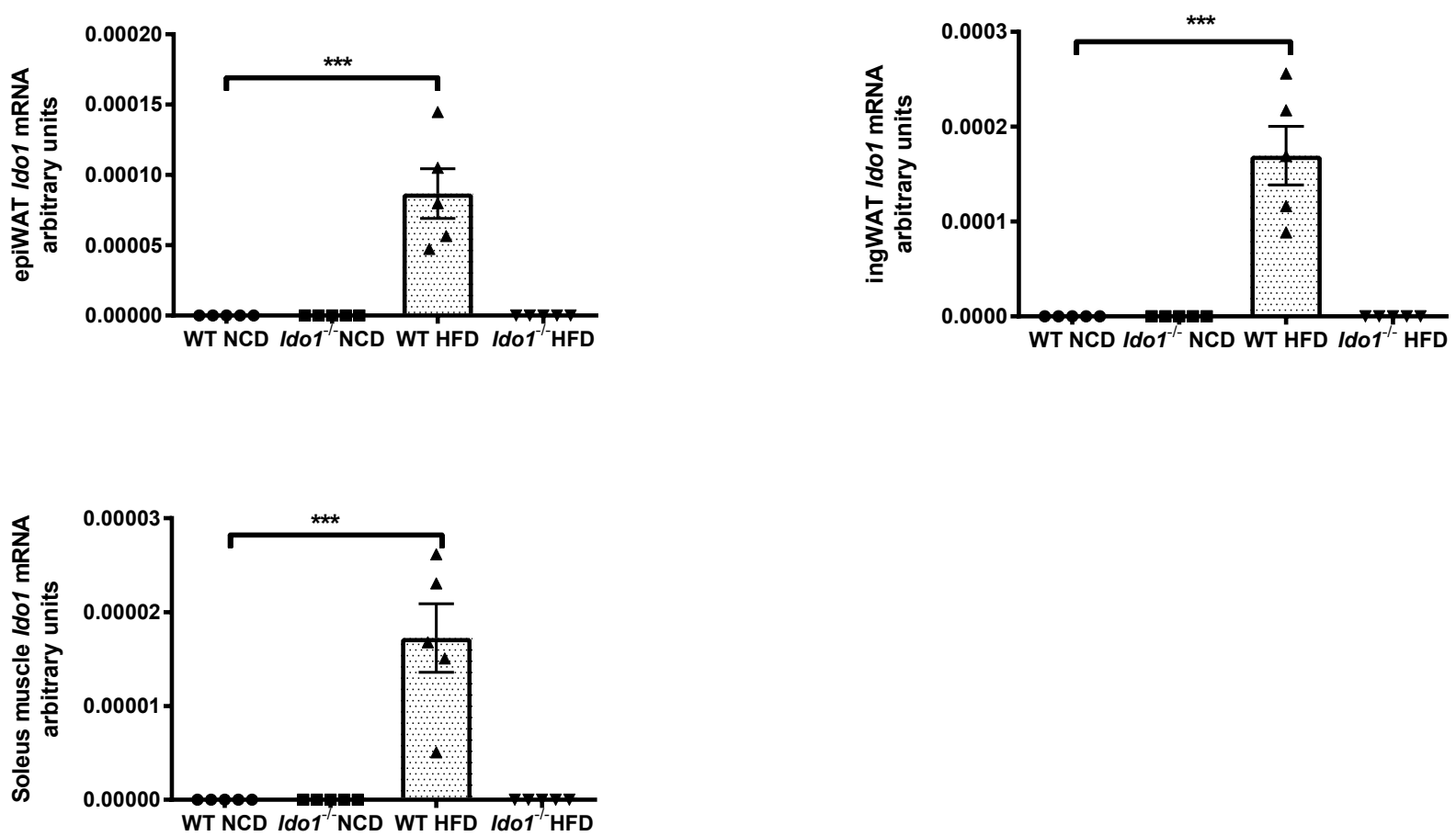

b
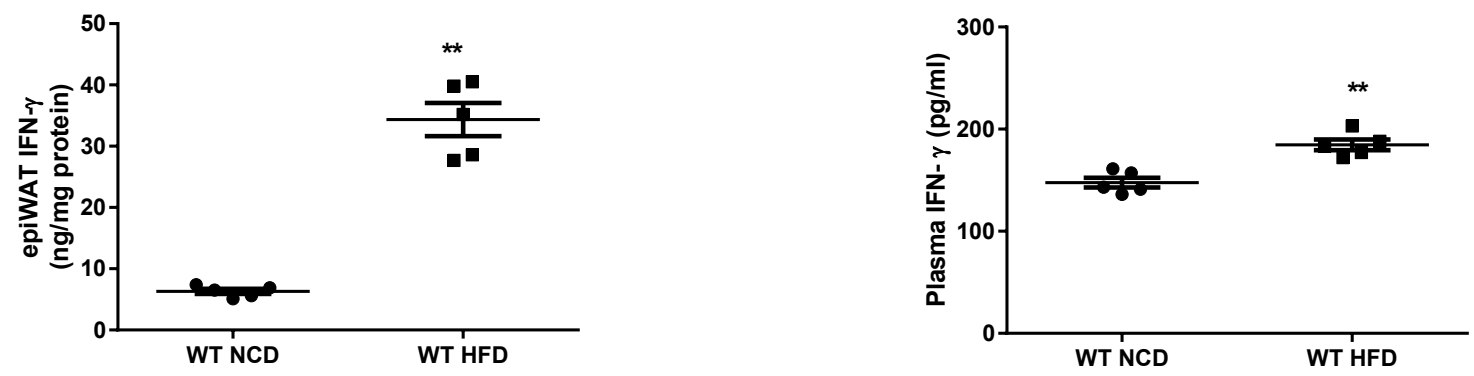
a

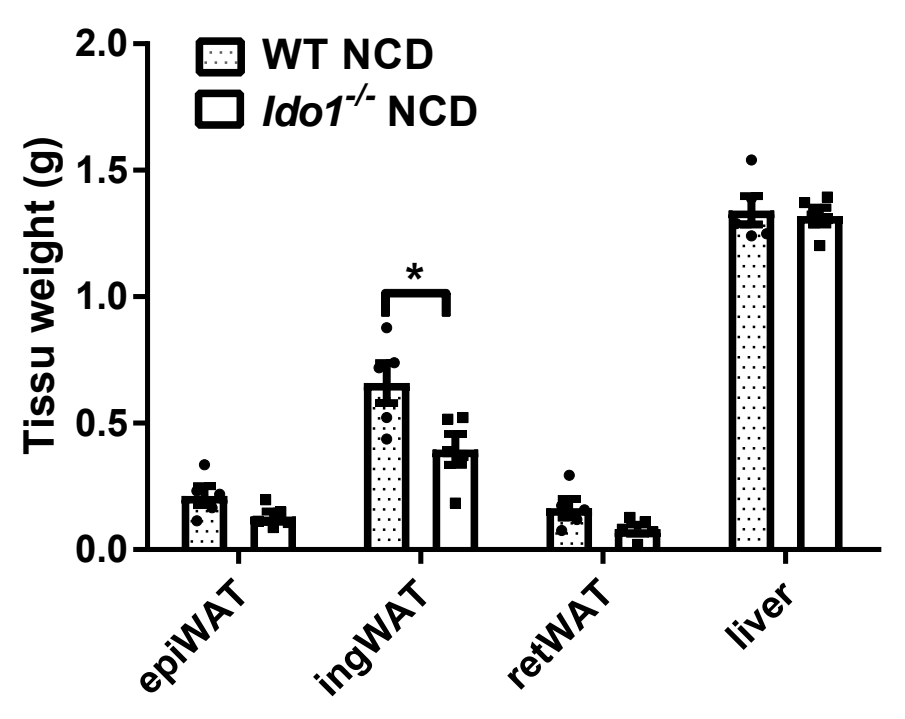

C

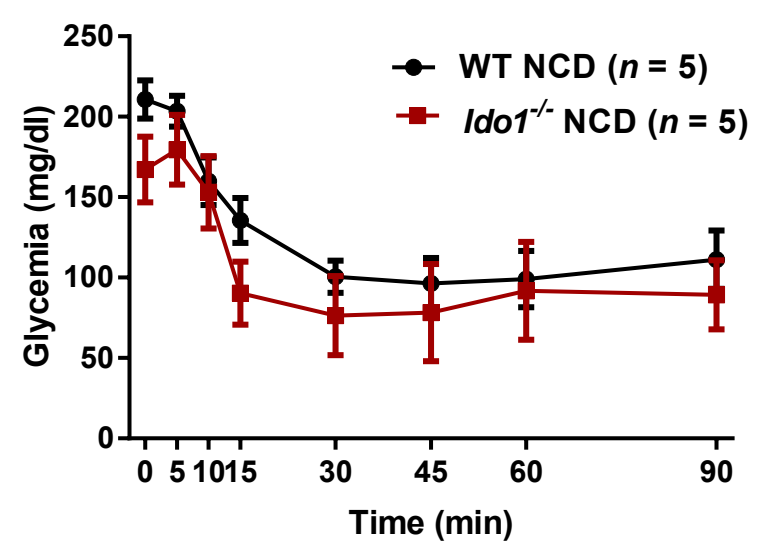

b

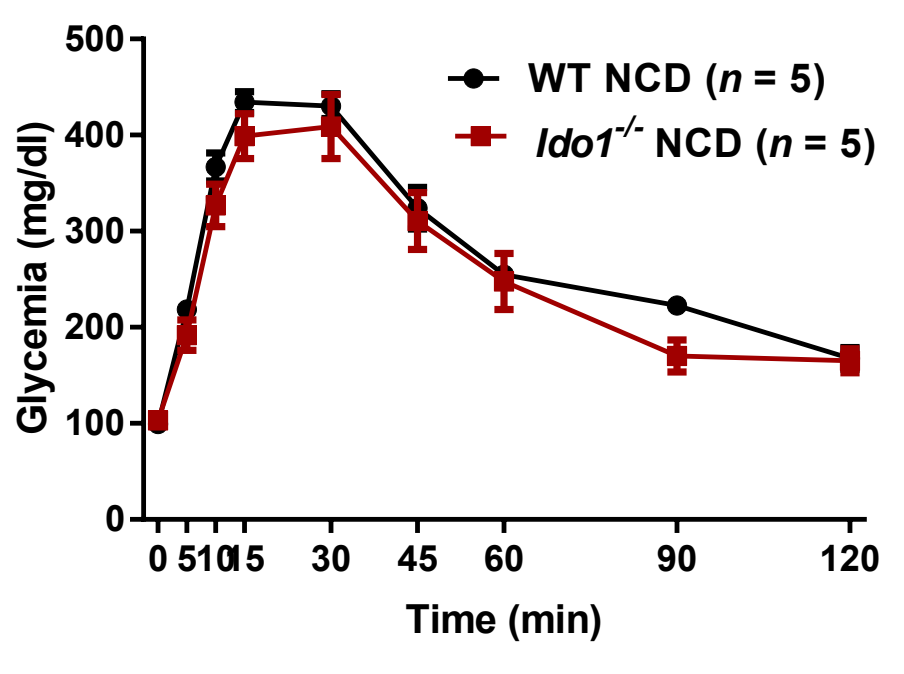

d

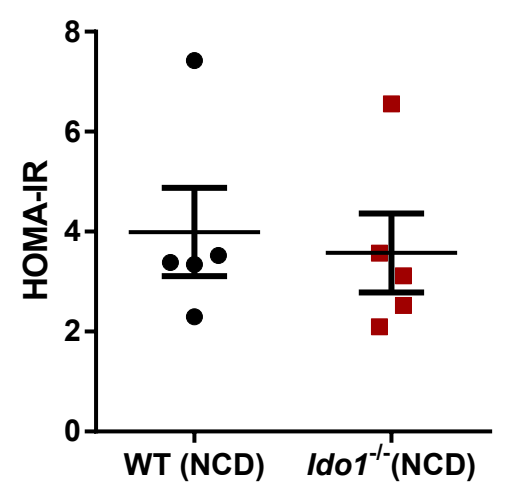




\section{Supplementary Fig 3}
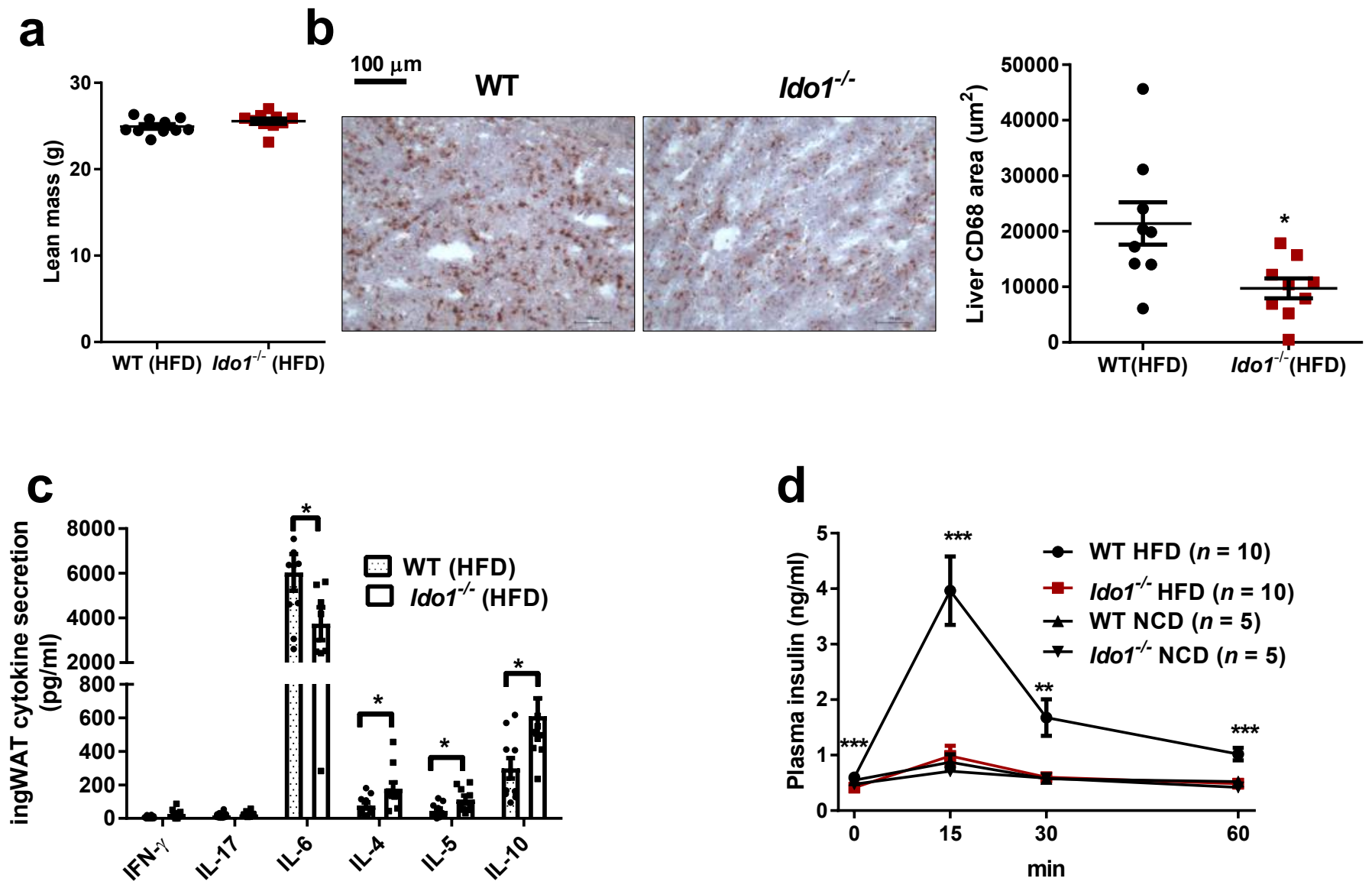

e
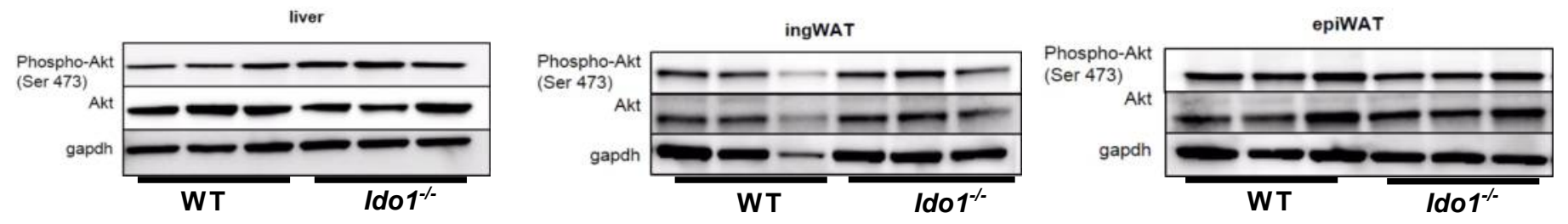

f

g

h
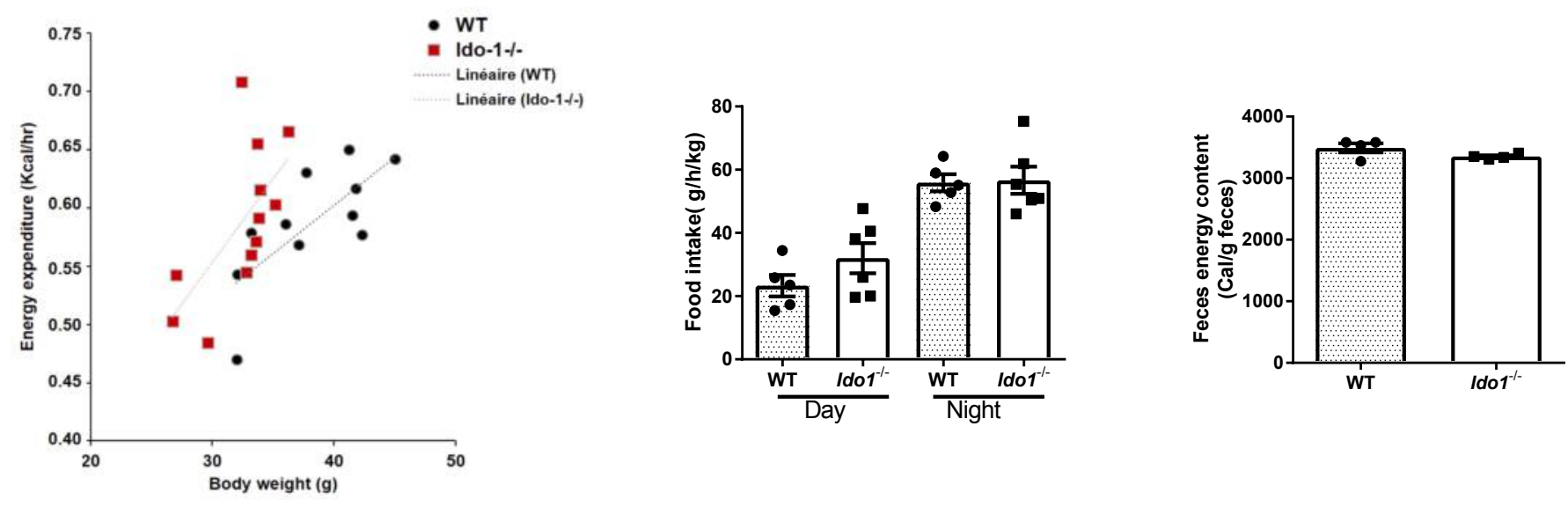
Supplementary Fig 4

a

WT $\quad I d o 1^{-/-}$
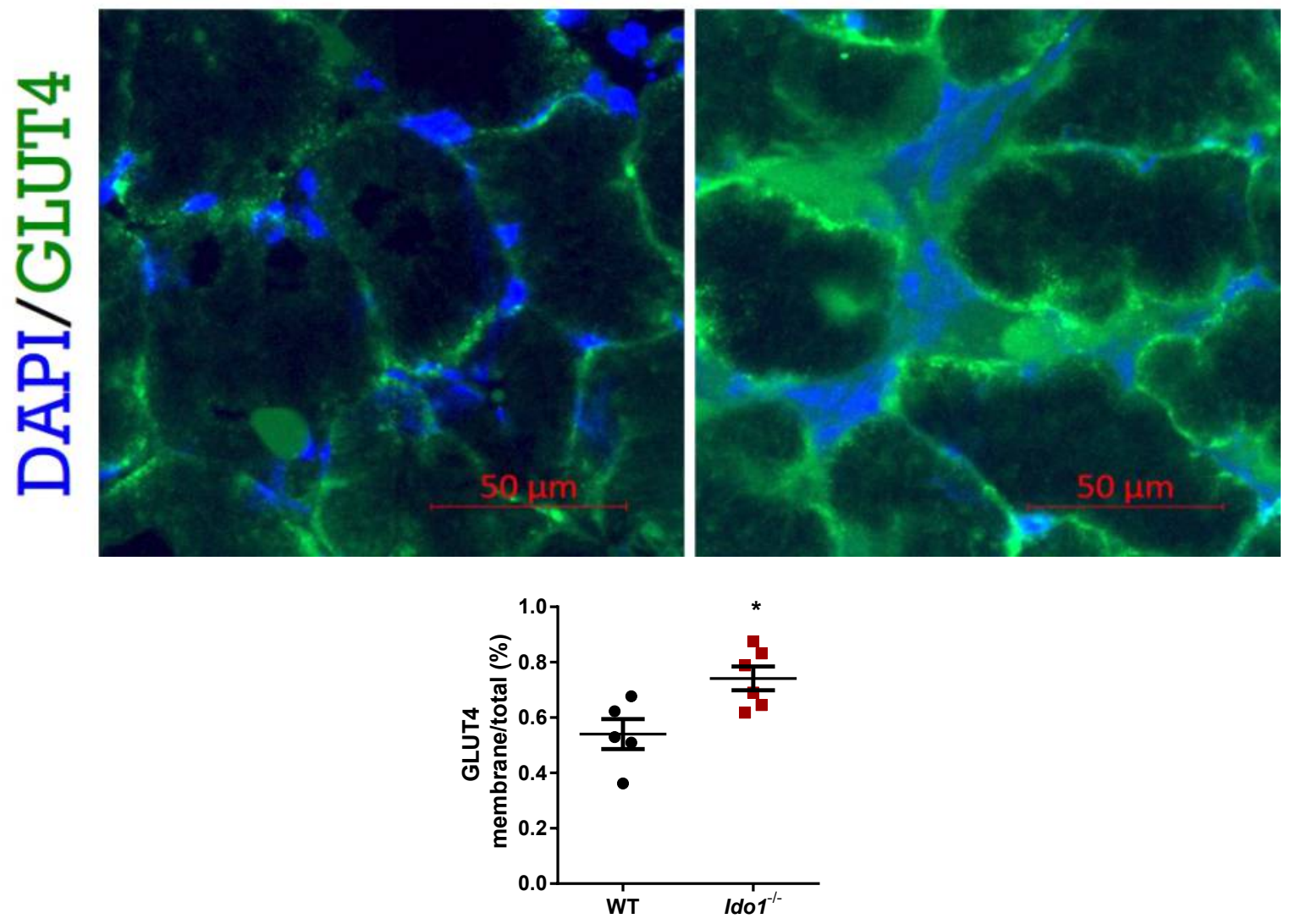

b

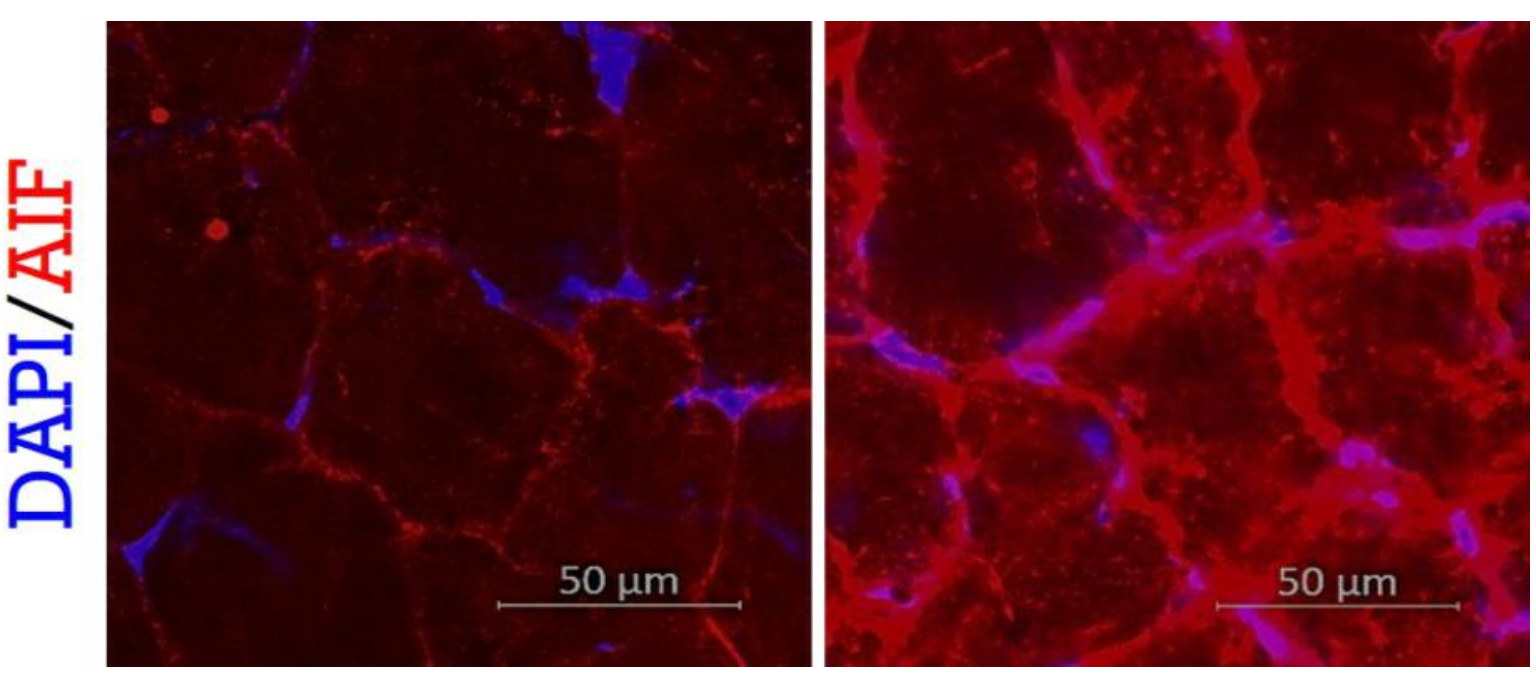

C
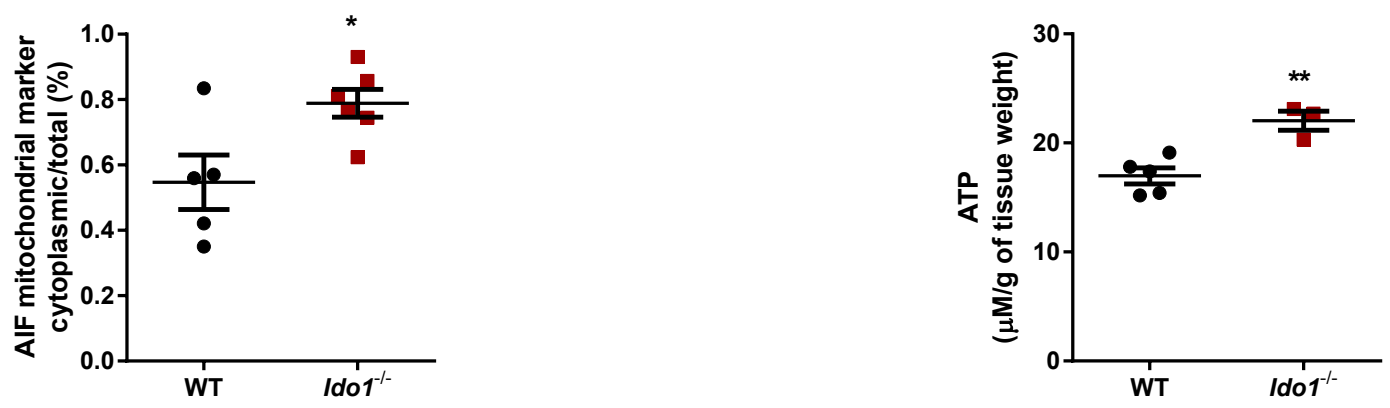


\section{Supplementary Fig 5}

a

b

\section{C}
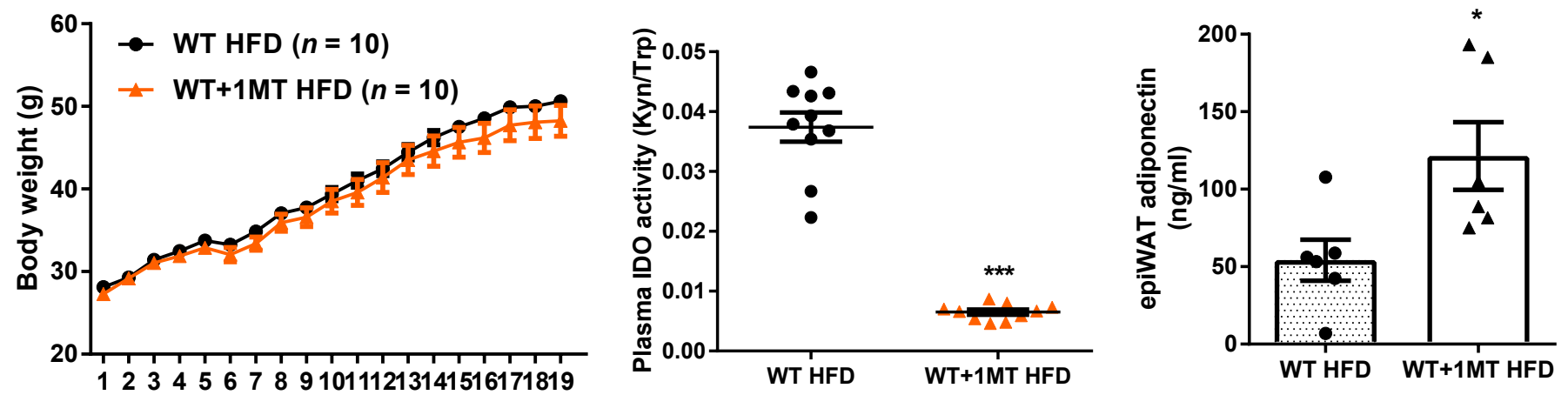

weeks of HFD

d

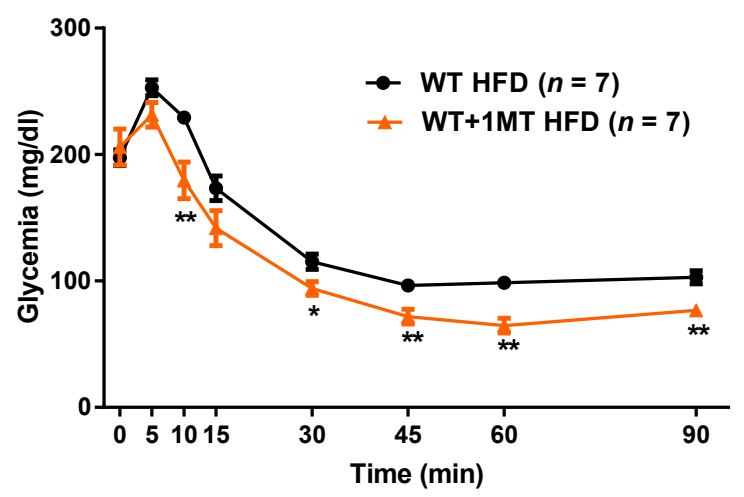

\section{f}

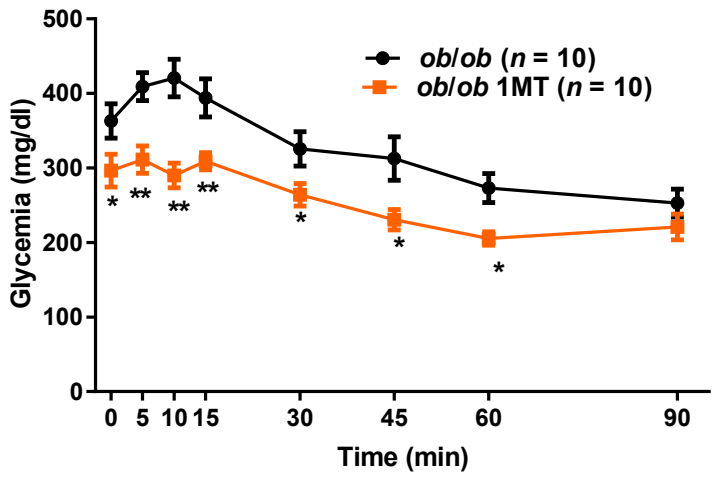

e

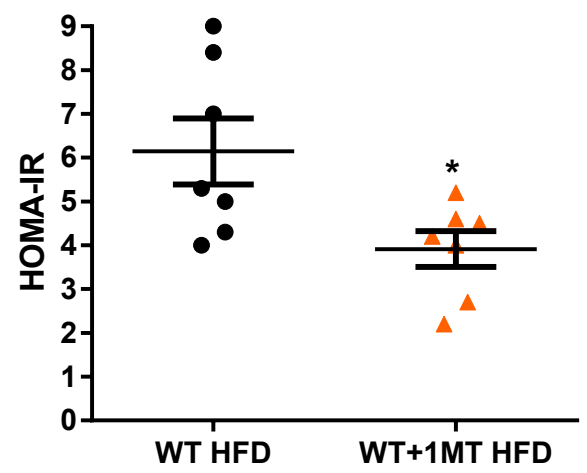

g

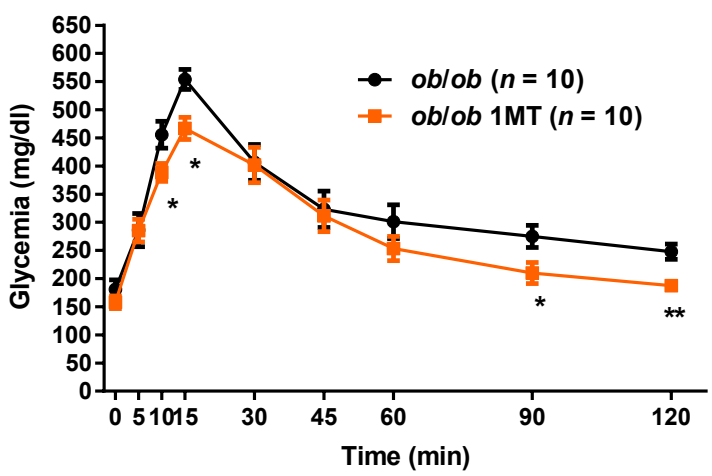




\section{Supplementary Fig 6}

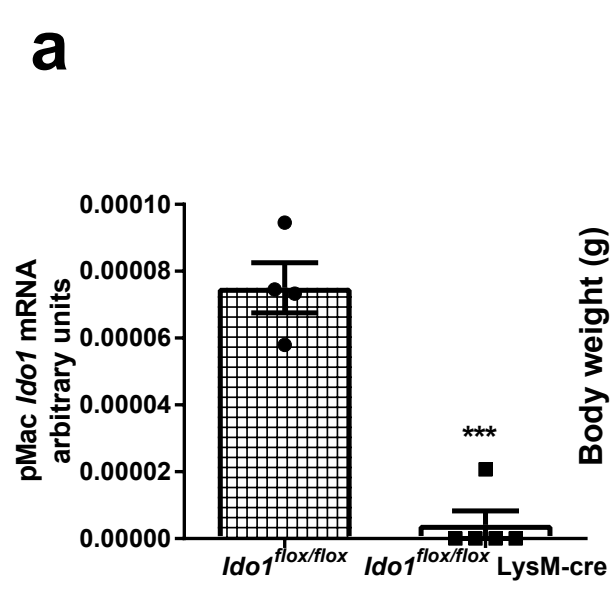

b

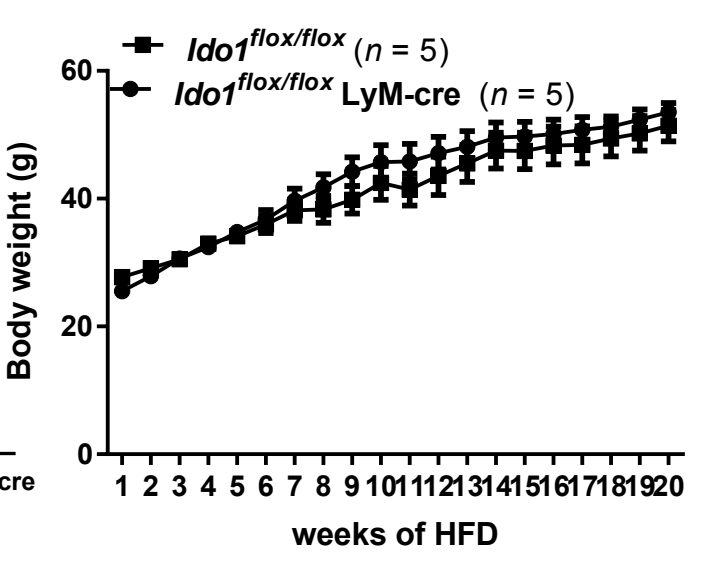

C

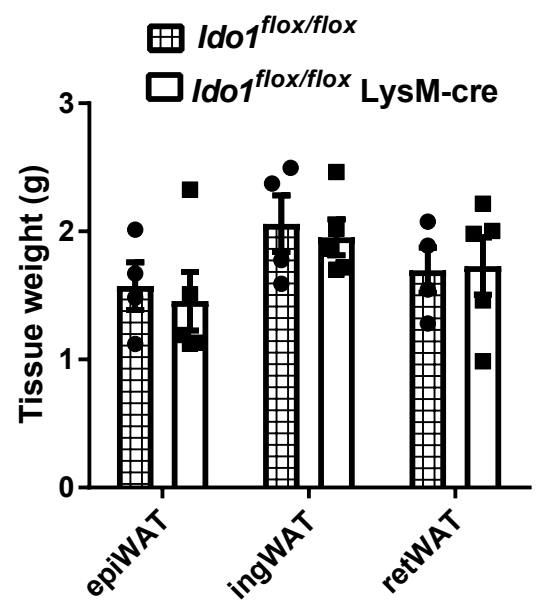

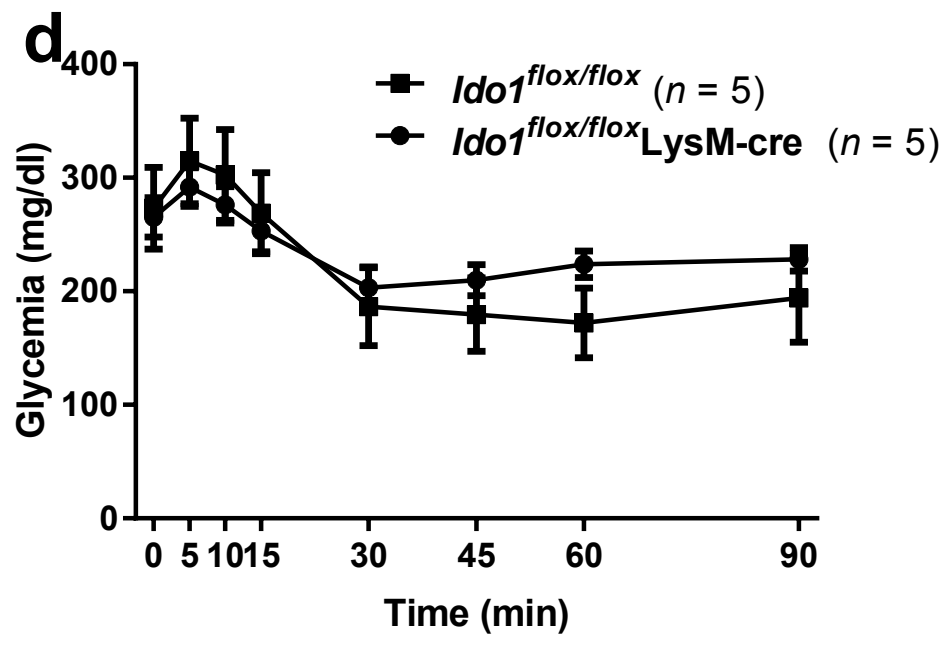

e

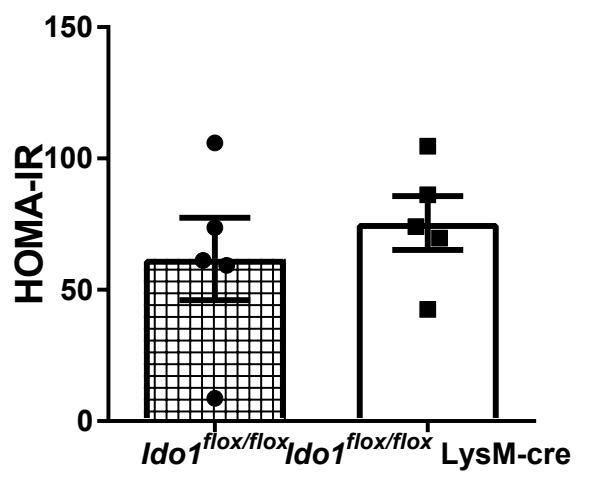


a

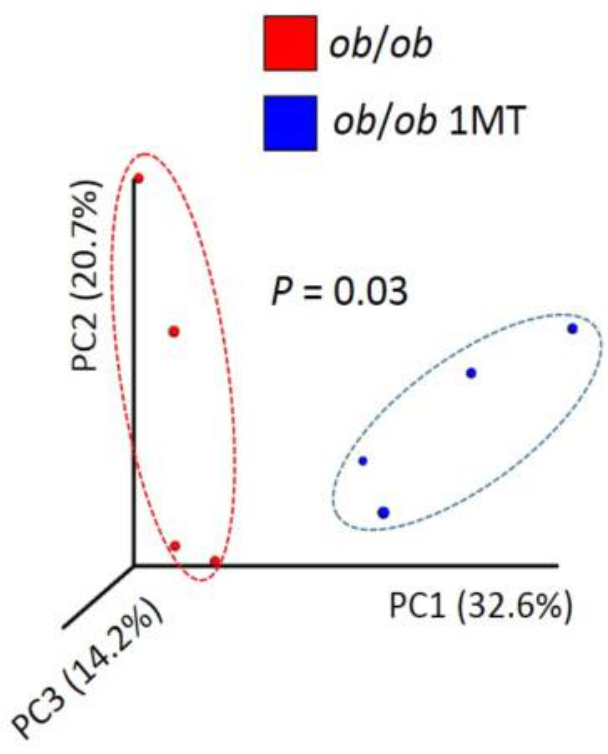

C

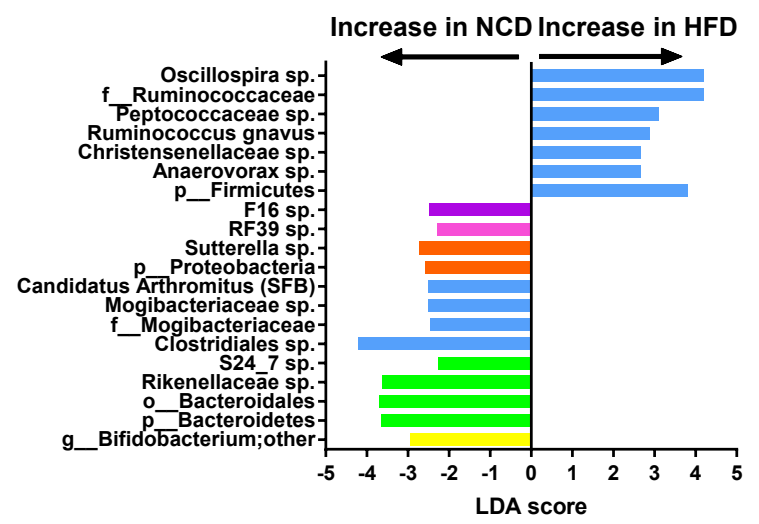

b

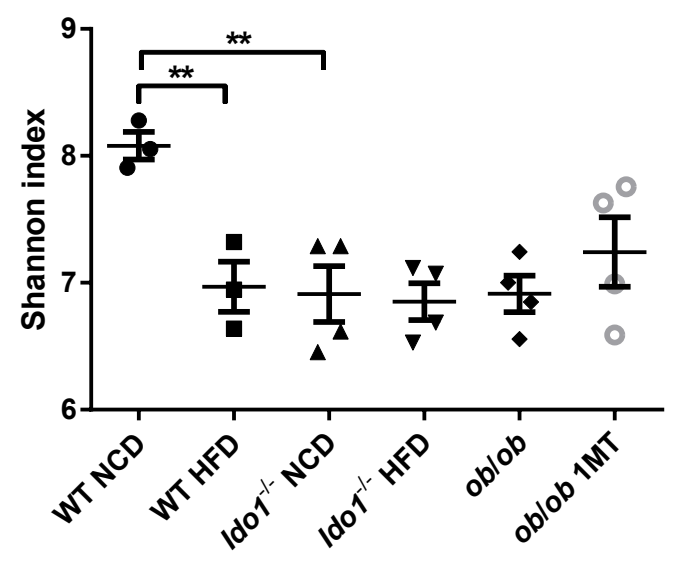

$I d o 1^{-/-}$

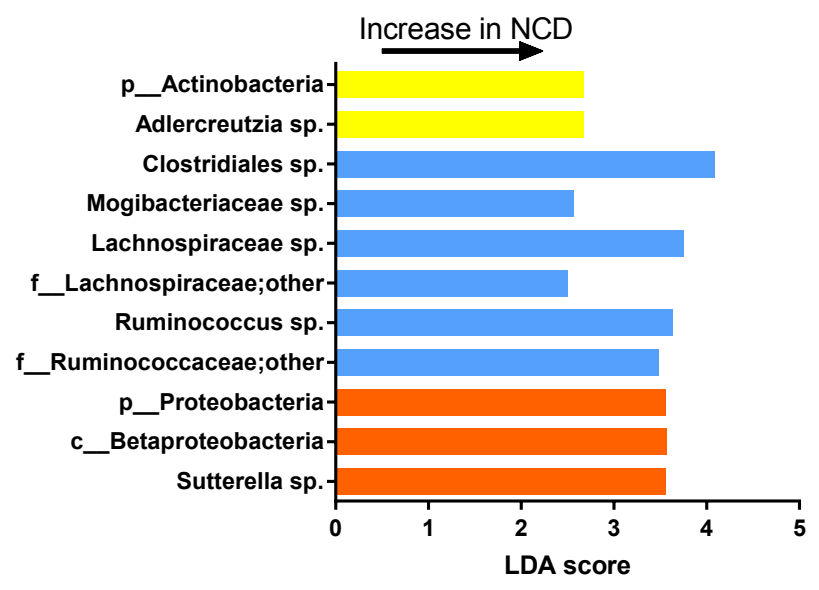

d

Family

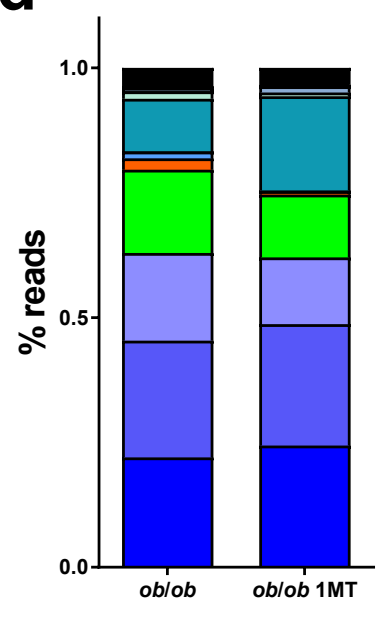

D f_Ruminococcaceae

$\square$ o_Clostridiales; $f$

$\square$ f_Lachnospiraceae

$\square$ f__Rikenellaceae

$\square f \_$Alcaligenaceae

$\square$ f_Erysipelotrichaceae

$\square$ f_Desulfovibrionaceae

$\square$ f_turicibacteraceae

$\square$ f_Clostridiaceae

$\square$ f__Peptococcaceae

$\square$ o_Clostridiales; Other

$\square$ f_Bifidobacteriaceae

$\square$ f_[Mogibacteriaceae]

$\square$ f__Peptostreptococcaceae

e

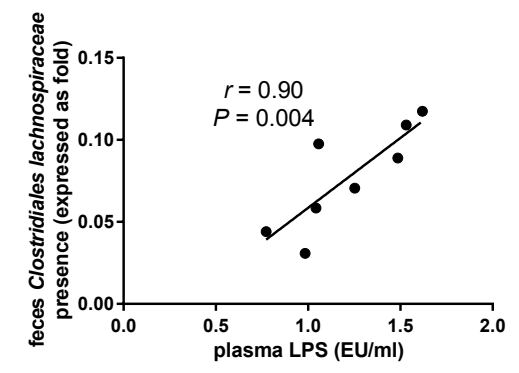

f

- other

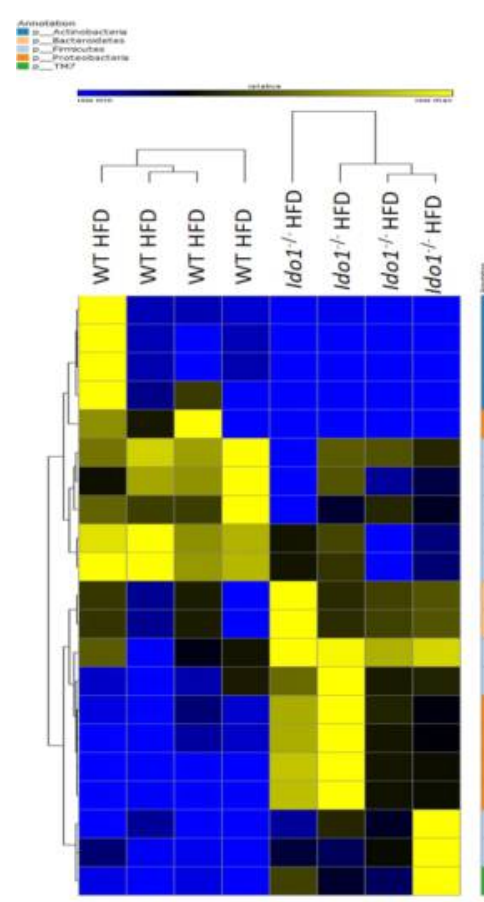




\section{Supplementary Fig 8}

a

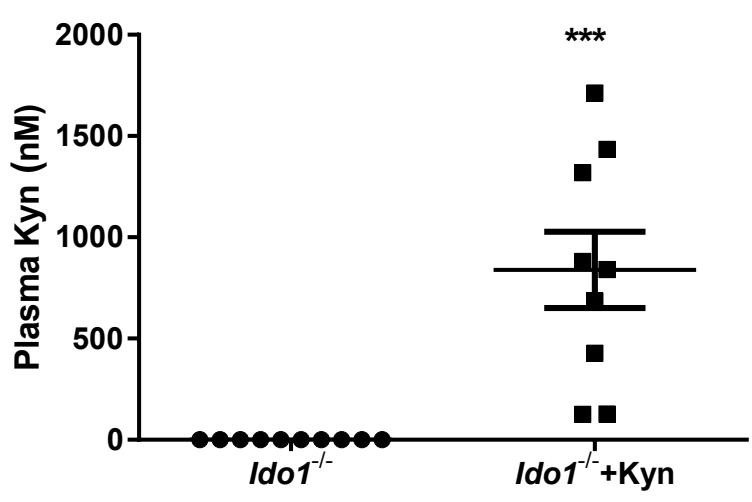

C

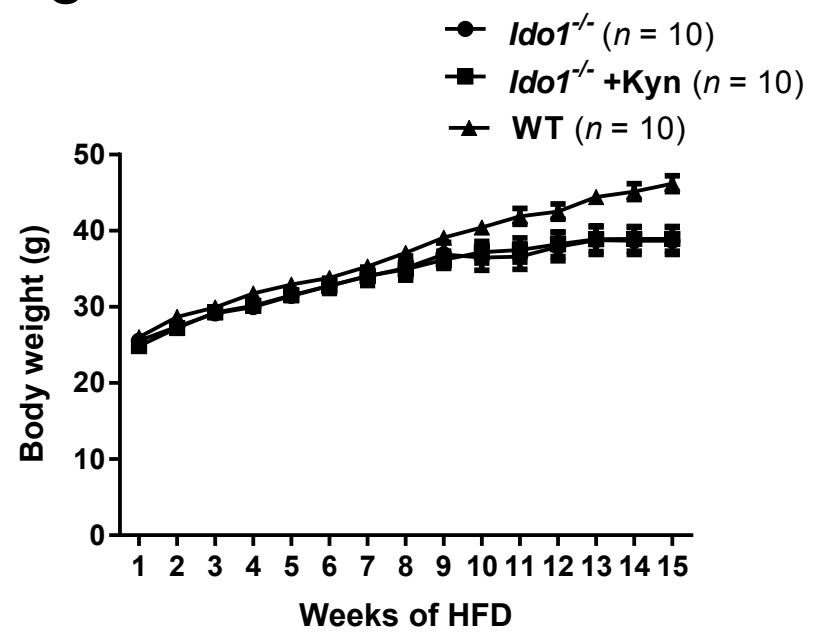

e

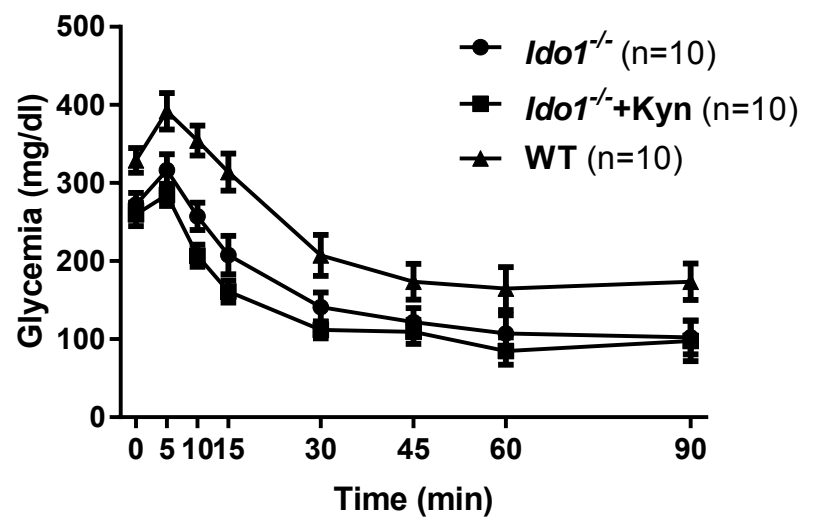

b

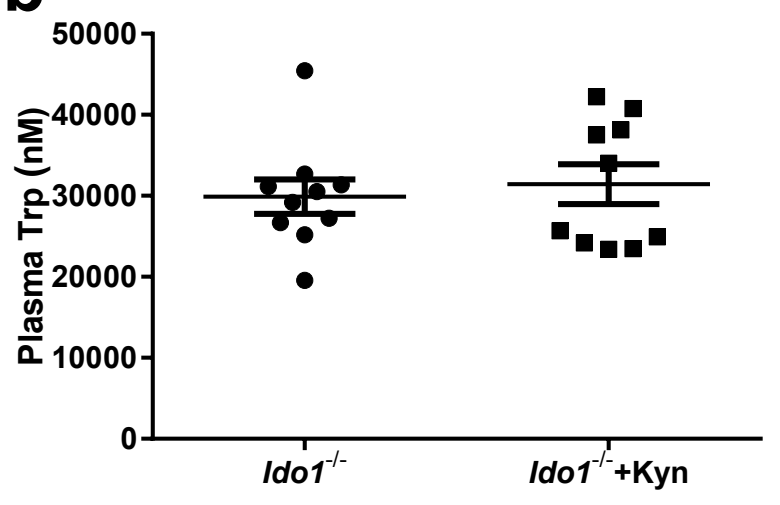

d

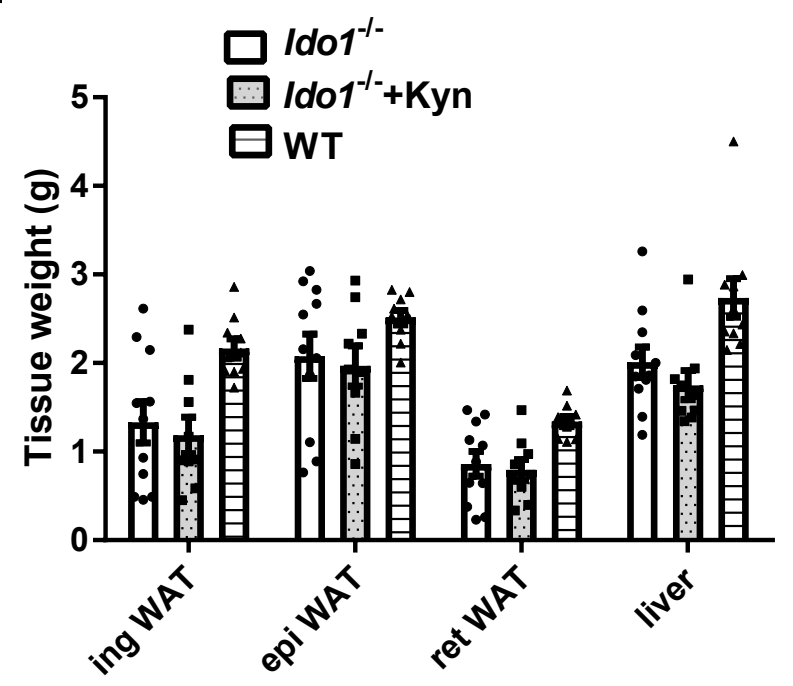

f

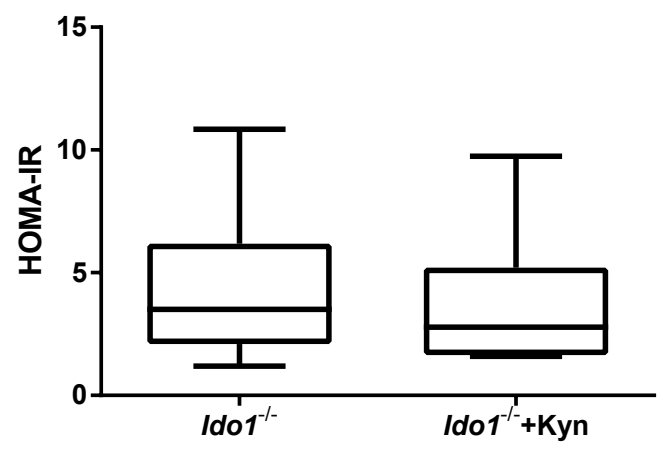


a
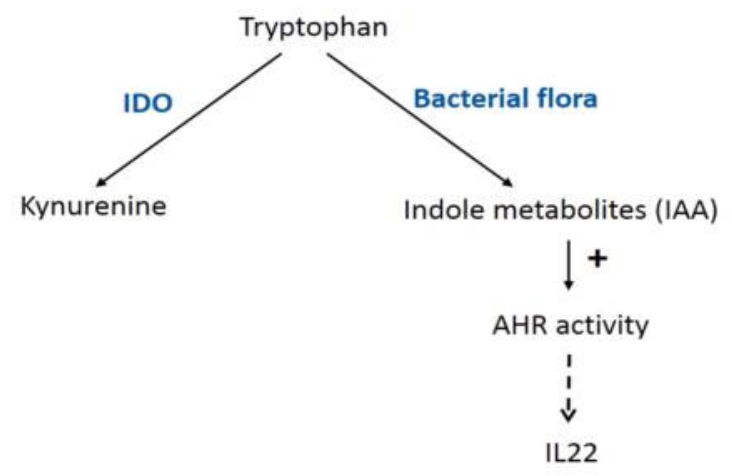

b

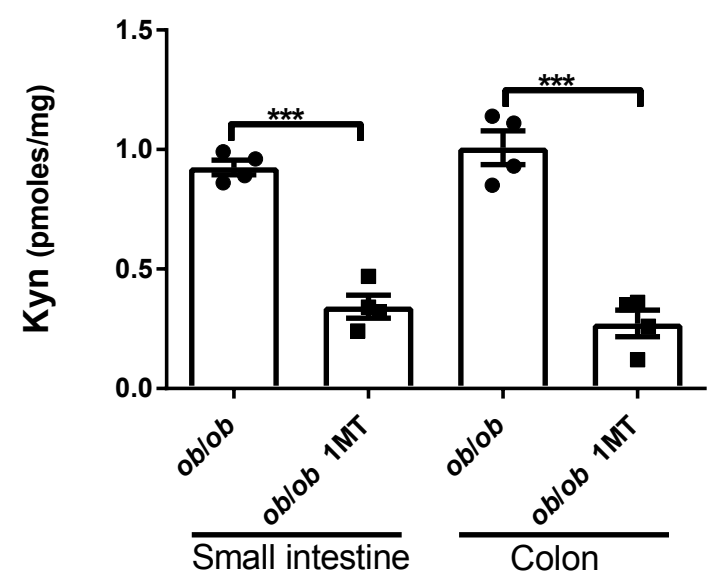

d

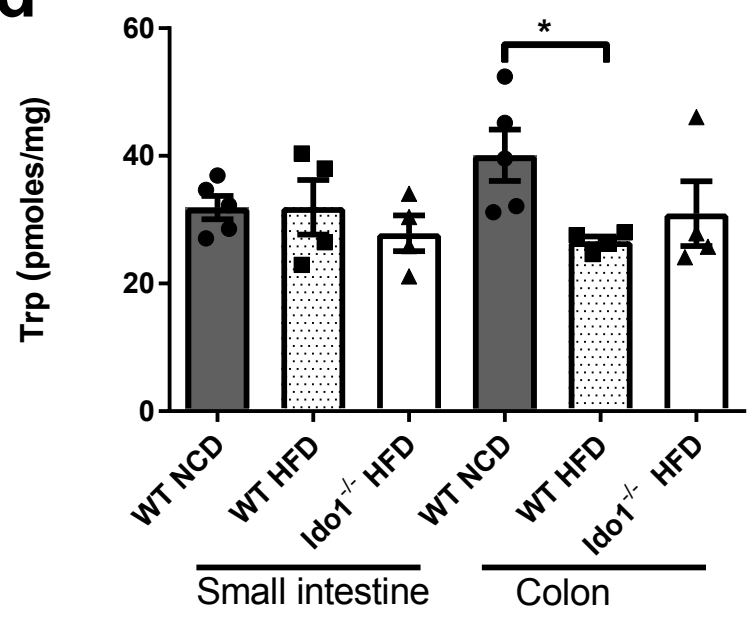

f
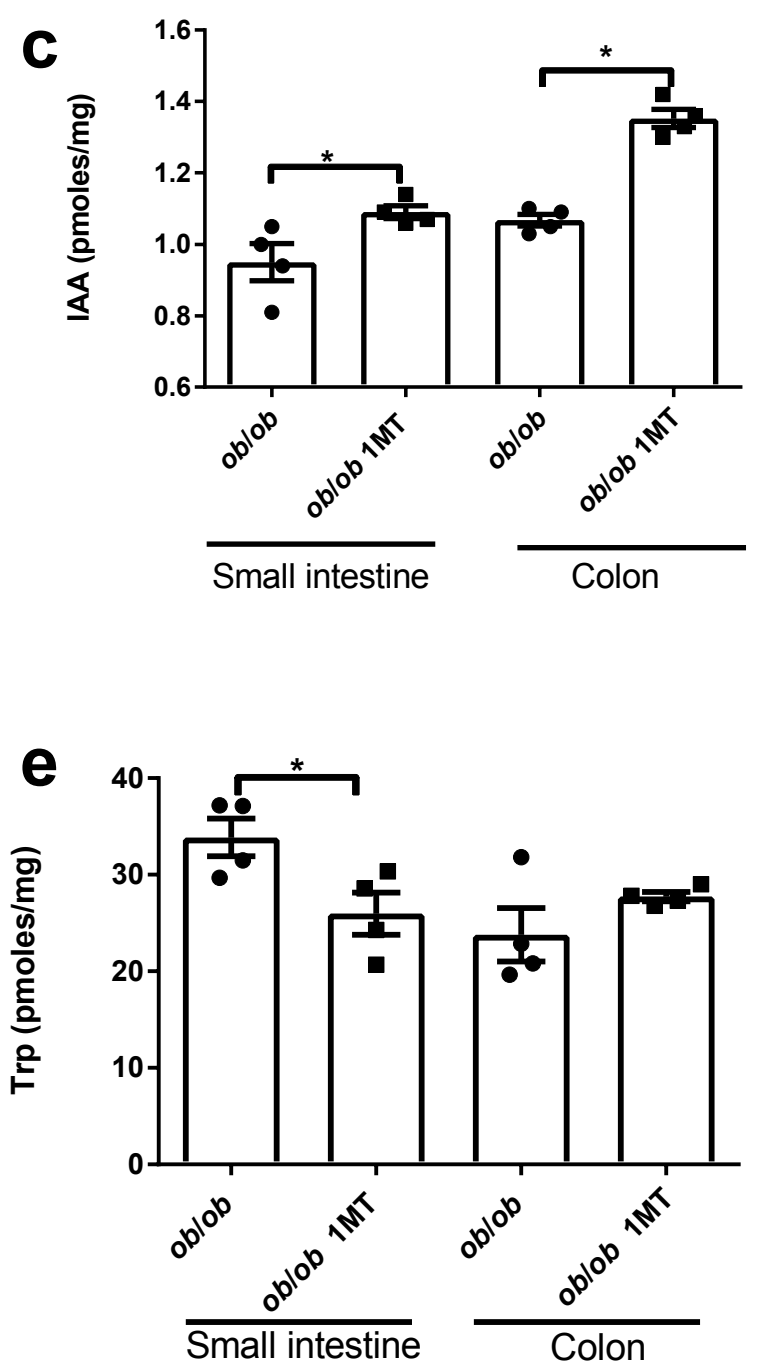

g

- WT (ob/ob feces)

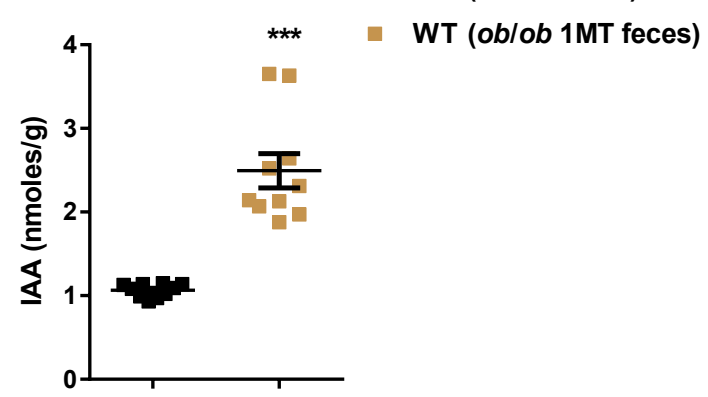

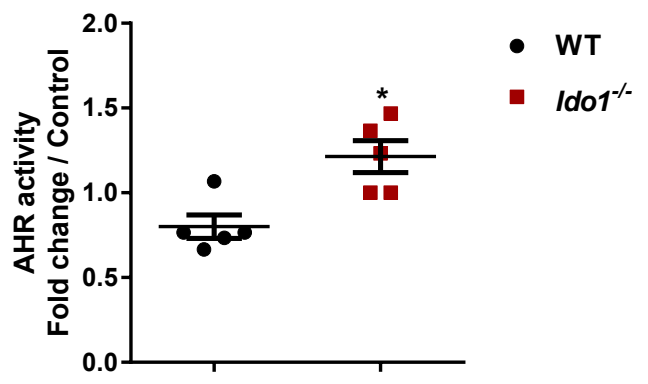




\section{Supplementary Fig. 10}

a

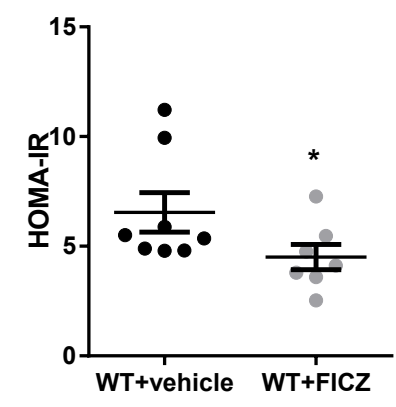

C

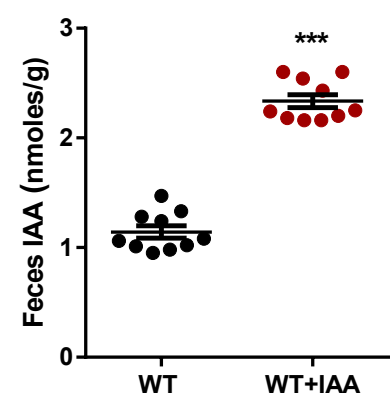

b
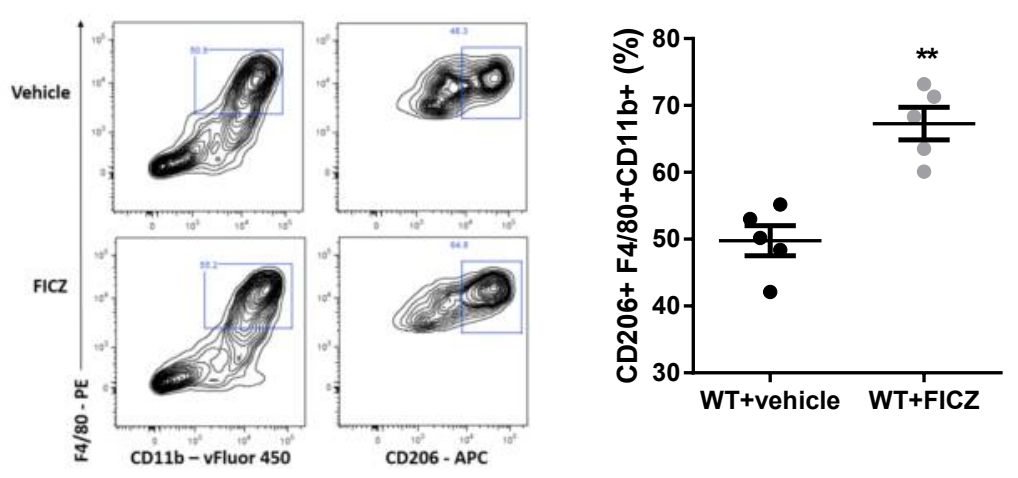

d

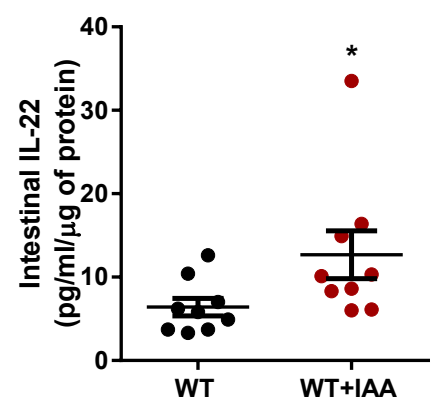

e

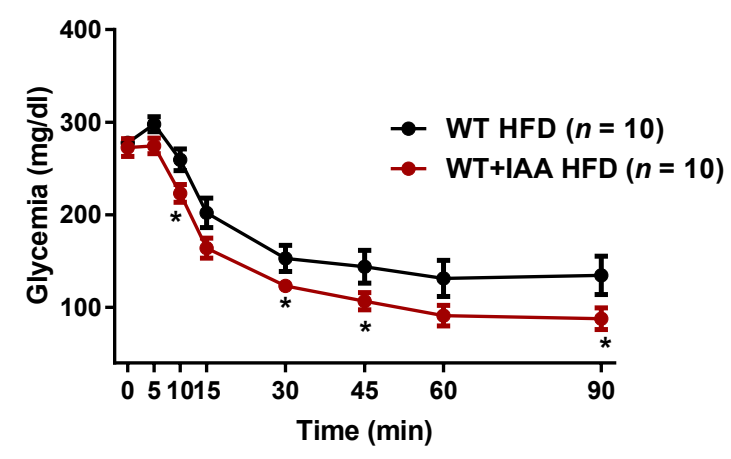

f

w
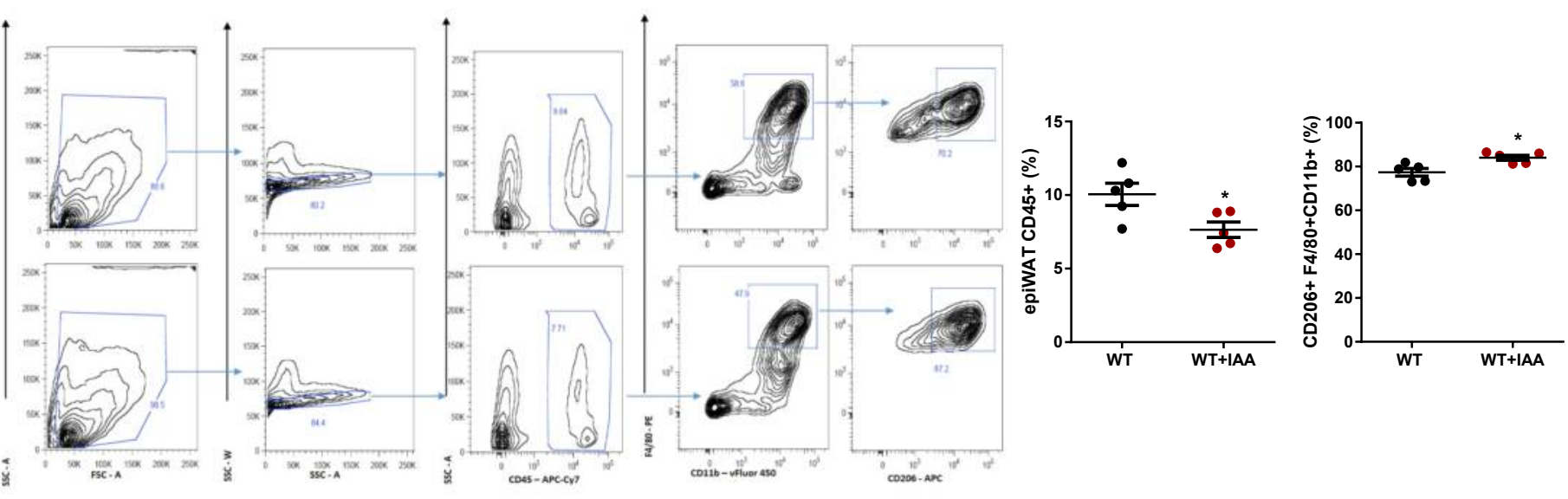

g

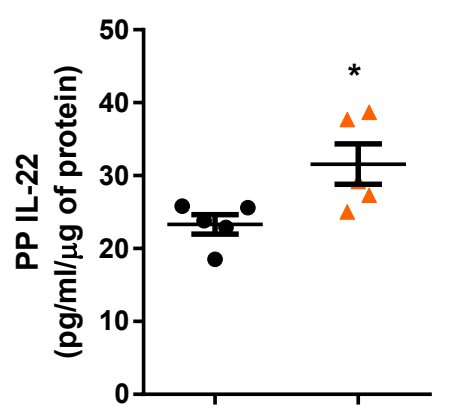

- WT HFD

\ WT+1MT HFD

i

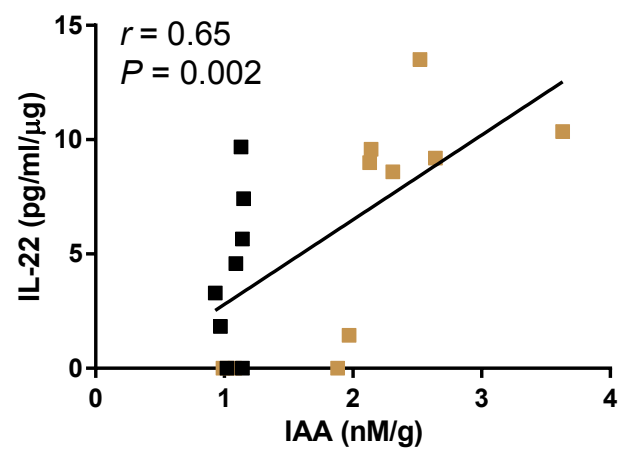

h

WT (ob/ob feces)
WT (ob/ob 1MT feces)

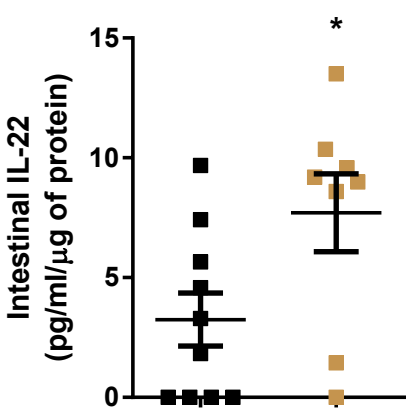

- WT (ob/ob feces)

WT (ob/ob 1MT feces)

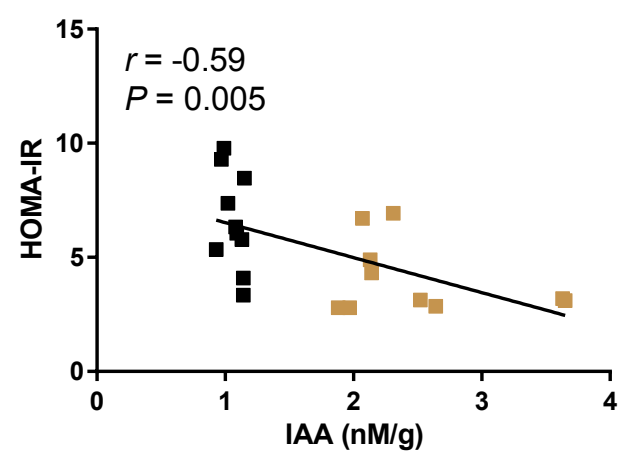


a

b
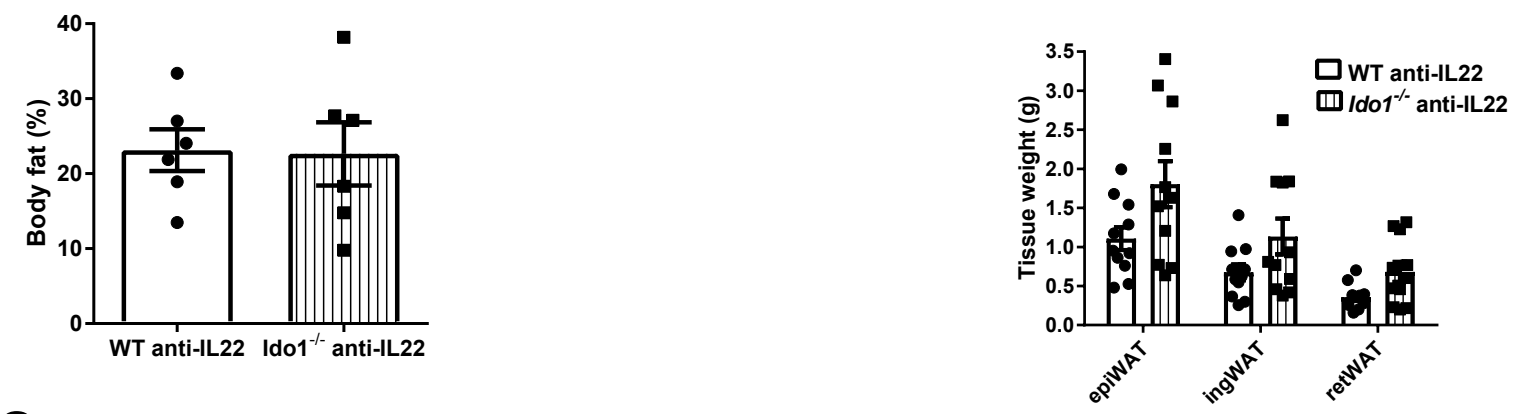

C

WT anti-IL22

Ido1/- anti-IL22
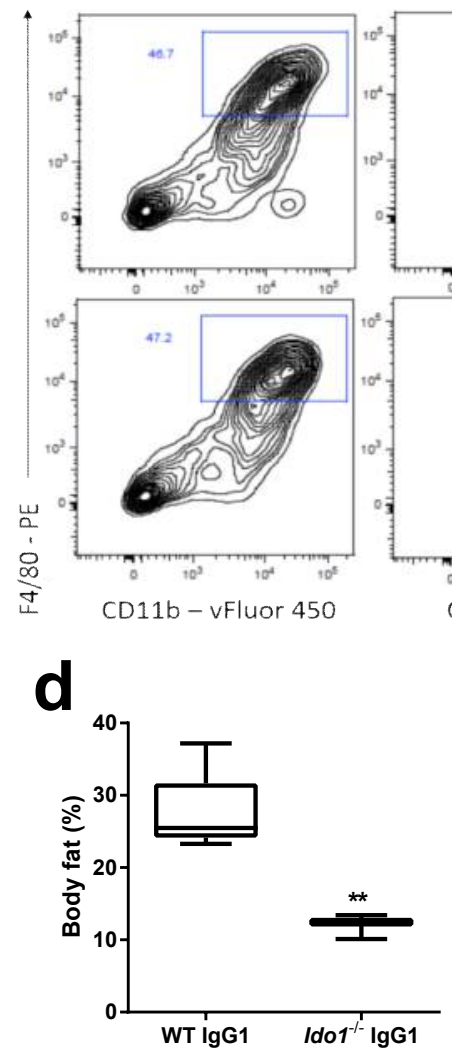
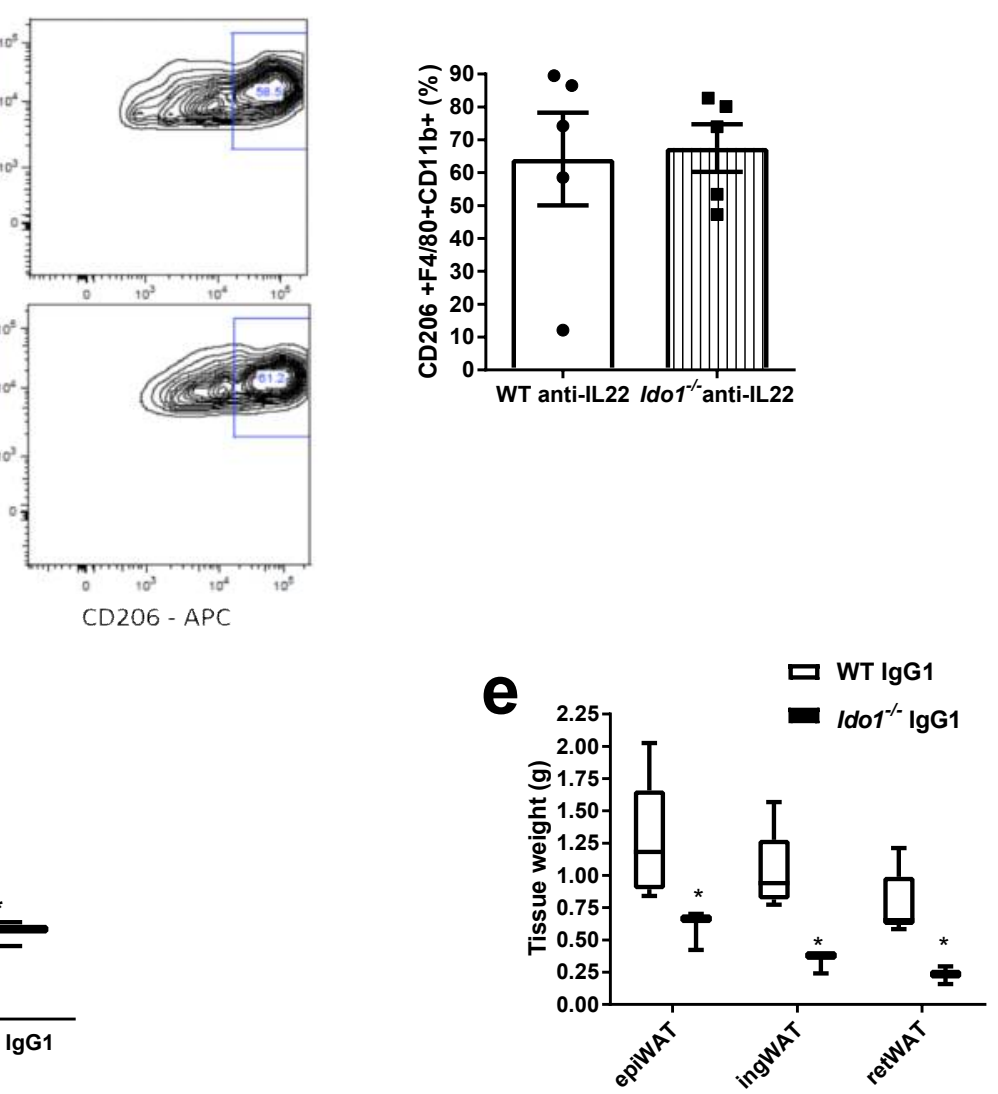

f

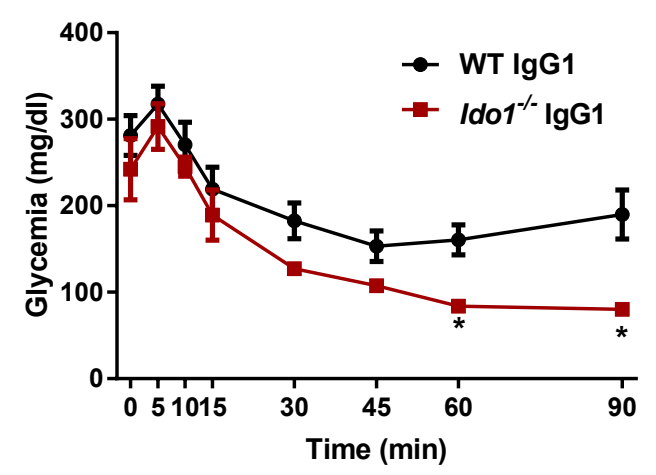

g

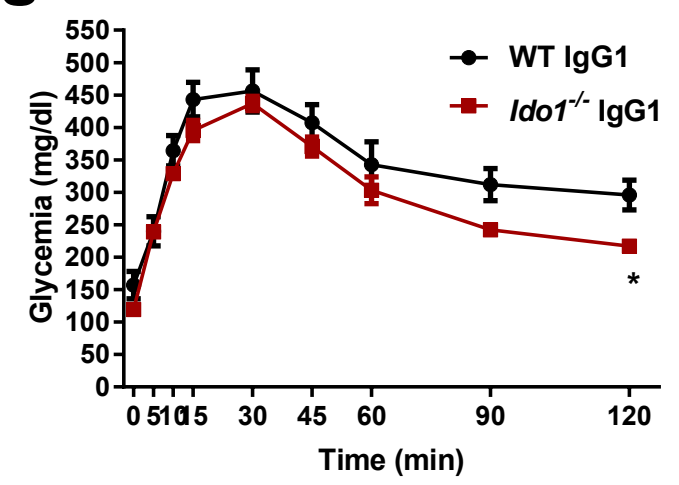

h

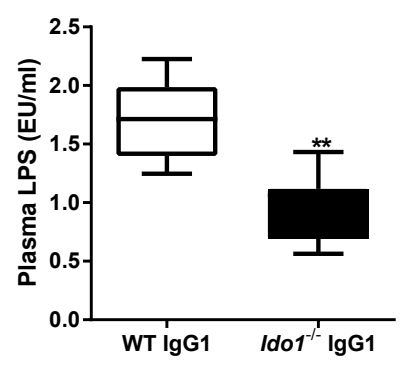


Supplementary Fig. 12

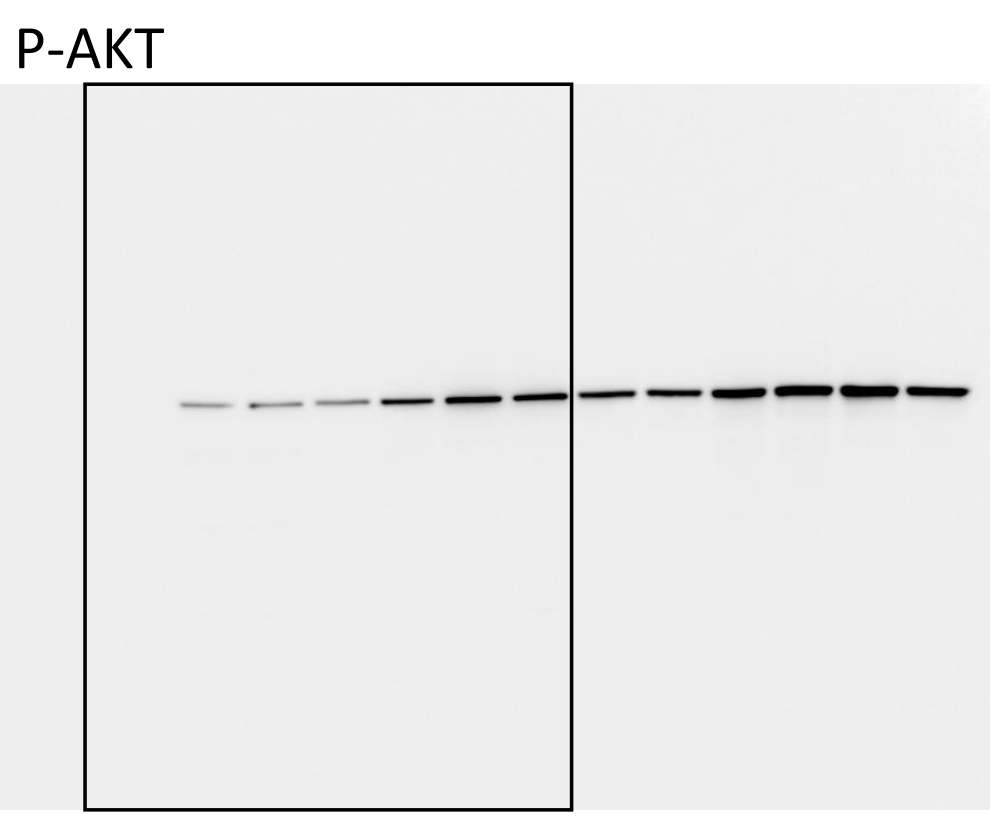

AKT

GAPDH

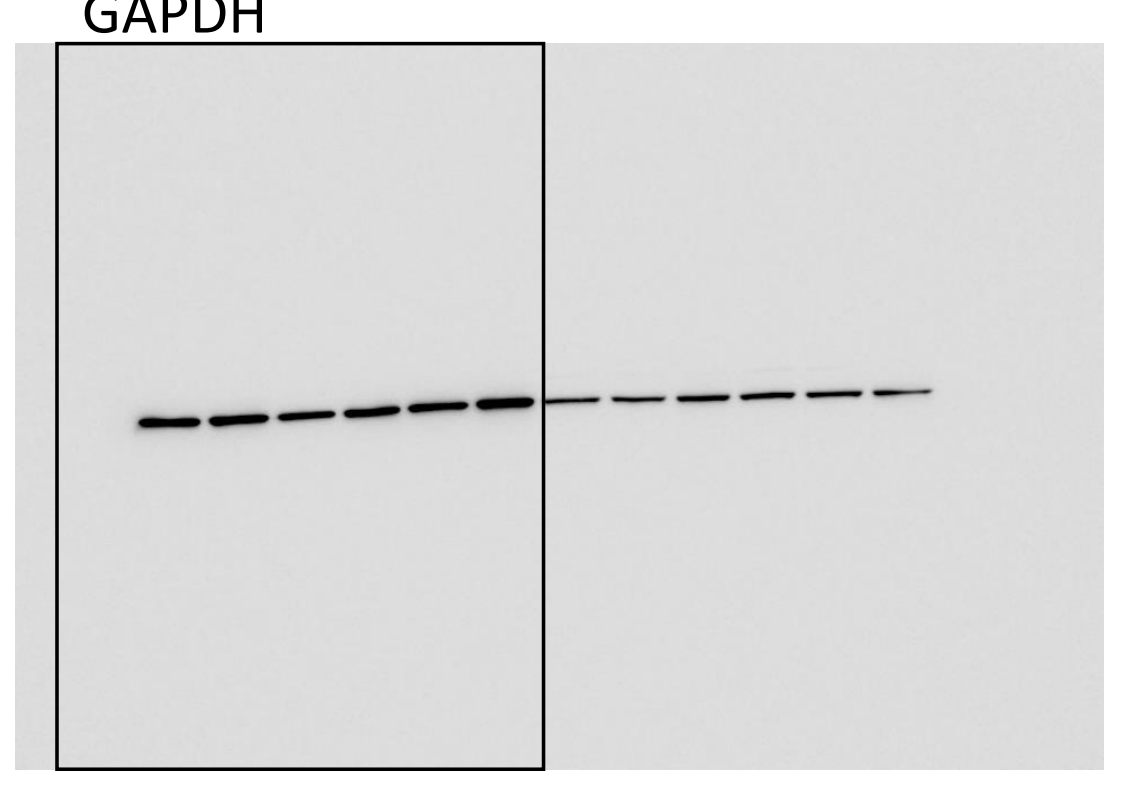

